Aus der Klinik für Gastroenterologie und gastrointestinale Onkologie

(Prof. Dr. med. V. Ellenrieder)

der Medizinischen Fakultät der Universität Göttingen

\title{
Differenzierung verschiedener Fettlebererkrankungen anhand laborchemischer und anamnestischer Parameter
}

\author{
INAUGURAL-DISSERTATION \\ zur Erlangung des Doktorgrades \\ der Medizinischen Fakultät der \\ Georg-August-Universität zu Göttingen
}

vorgelegt von

Bernd Rensing

aus

Salzkotten

Göttingen 2020 
Dekan:

\section{Betreuungsausschuss}

Betreuer:

Ko-Betreuer:

\section{Prüfungskommission}

Referent/in:

Ko-Referent/in:

Drittreferent/in:

Datum der mündlichen Prüfung: 02.09.2021
PD Dr. med. S. Kunsch

PD Dr. med. T. Lorf
PD Dr. Thomas Lorf

Prof Dr. Thomas Meyer 
Hiermit erkläre ich, die Dissertation mit dem Titel "Differenzierung verschiedener Fettlebererkrankungen anhand laborchemischer und anamnestischer Parameter" eigenständig angefertigt und keine anderen als die von mir angegebenen Quellen und Hilfsmittel verwendet zu haben.

Göttingen, den 


\section{Inhaltsverzeichnis}

Abbildungsverzeichnis .................................................................................. III

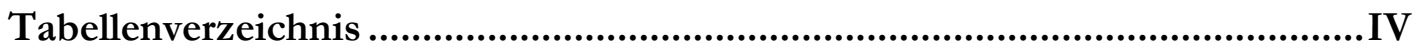

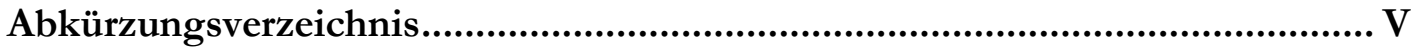

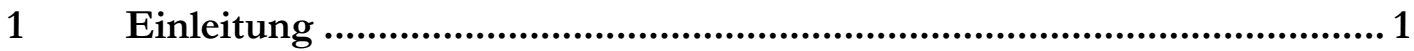

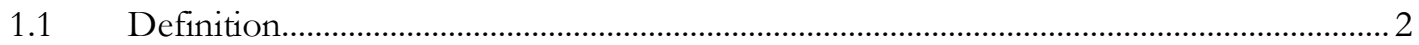

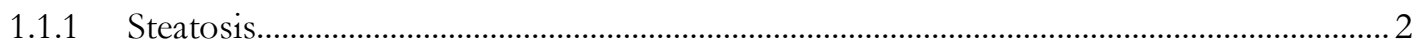

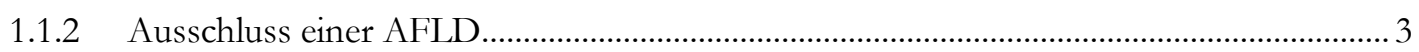

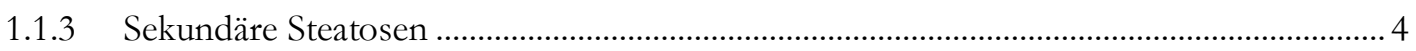

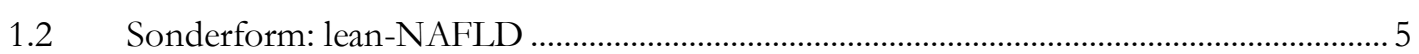

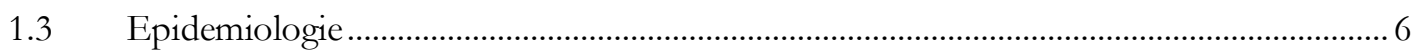

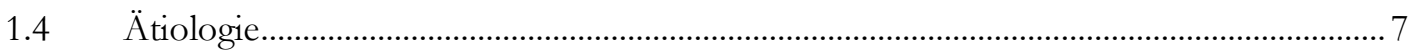

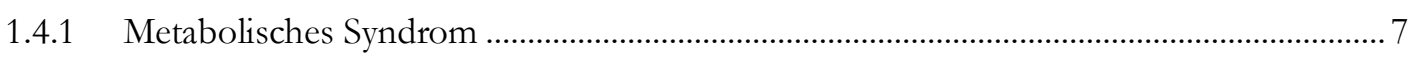

1.4.2 Suchtmittel/Lifestyle ..............................................................................................

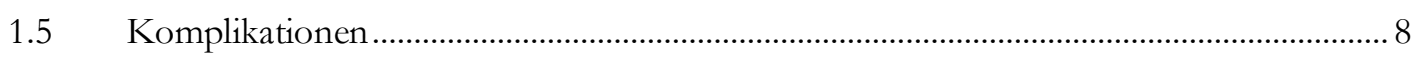

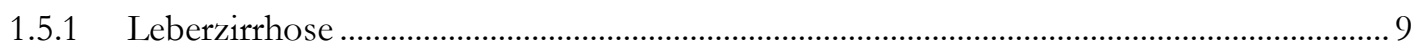

1.5.2 Hepatozelluläres Karzinom ..................................................................................

1.5.3 Notwendigkeit einer Lebertransplantation...............................................................

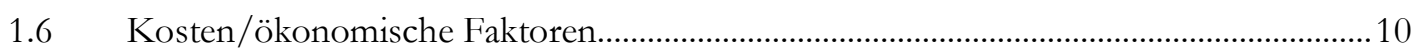

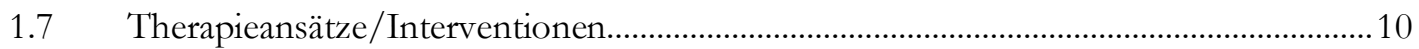

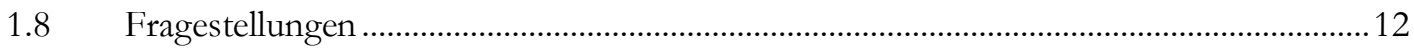

1.8.1 Differenzierbarkeit von AFLD und NAFLD anhand klinischer Routineparameter ....12

1.8.2 Kurzzeit-Follow-up der lean-NAFLD im Vergleich zur non-lean-NAFLD...................12

1.8.3 Effekte einer standardisierten Kurzberatung im Kurzzeitintervall ..................................12

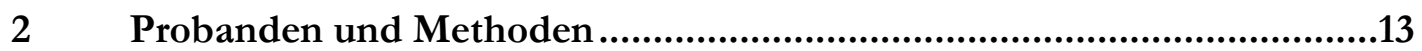

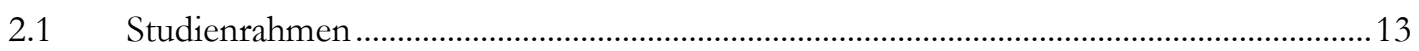

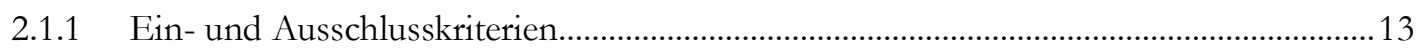

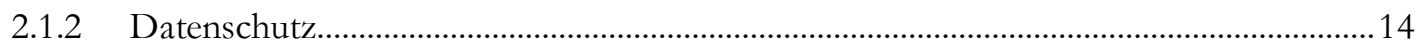

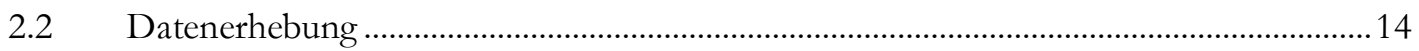

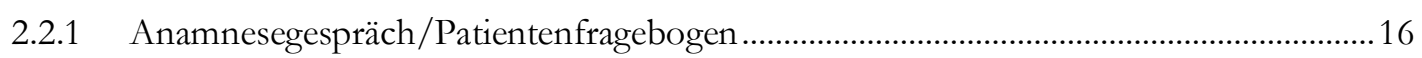

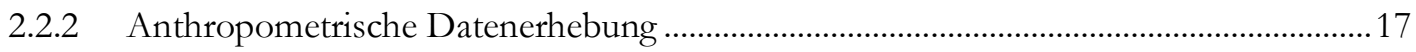

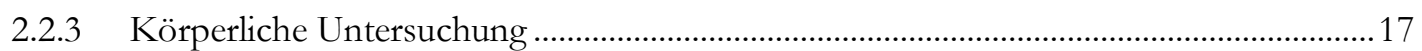

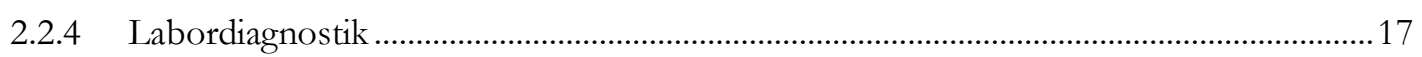

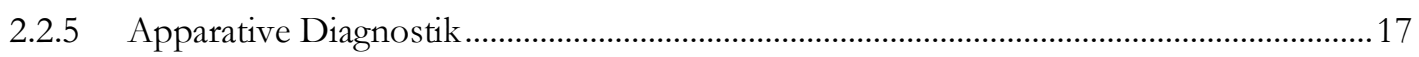

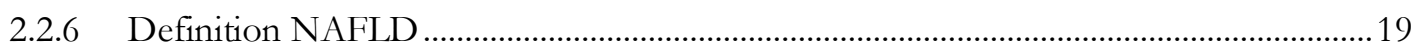

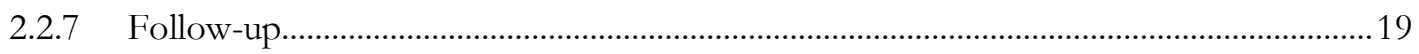

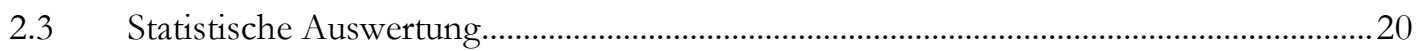

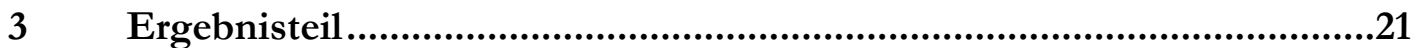




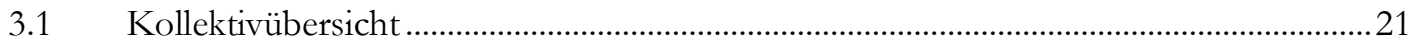

3.2 Differenzierbarkeit von AFLD und NAFLD anhand klinischer Routineparameter ....23

3.2.1 Epidemiologische und anthropometrische Charakteristika des Kollektivs ...................23

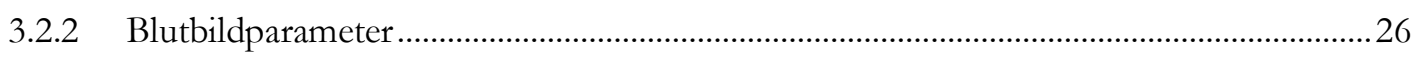

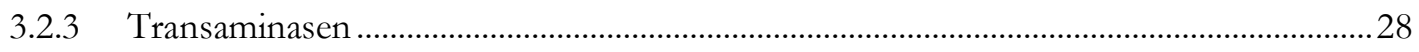

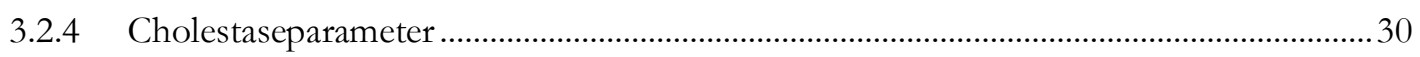

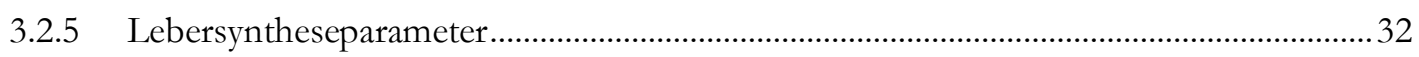

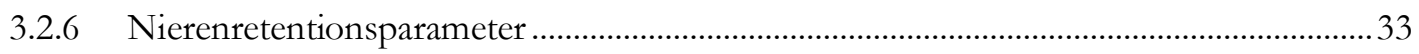

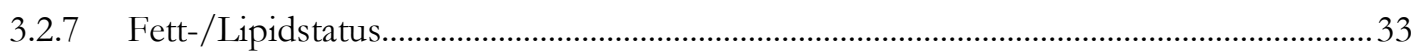

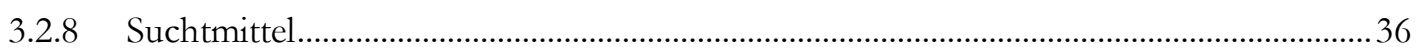

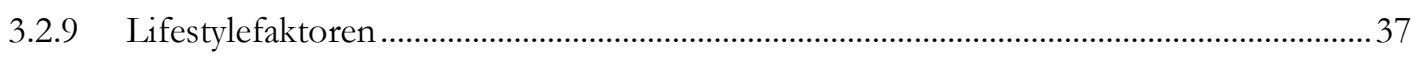

3.3 Kurzzeit-Follow-up der lean-NAFLD im Vergleich zur non-lean-NAFLD..................39

3.4 Effekte einer standardisierten Kurzberatung im Kurzzeitintervall .................................44

$4 \quad$ Diskussion...................................................................................... 47

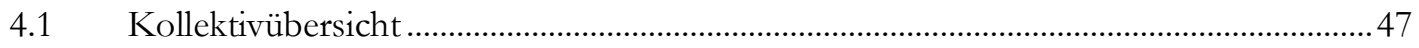

4.2 Differenzierbarkeit von AFLD und NAFLD anhand klinischer Routineparameter .... 47

4.2.1 Epidemiologische und anthropometrische Charakteristika des Kollektivs ................... 47

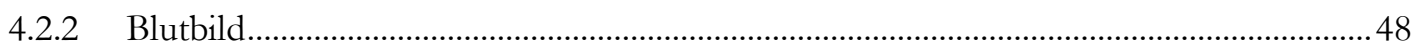

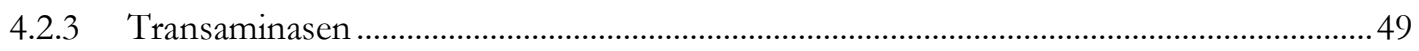

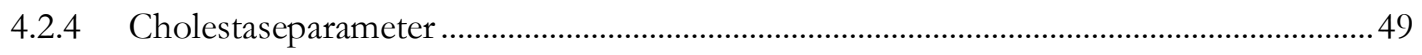

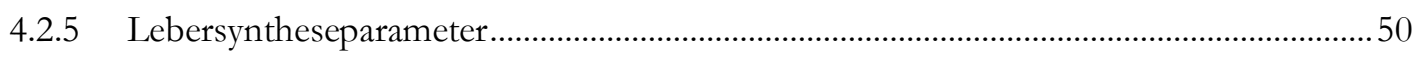

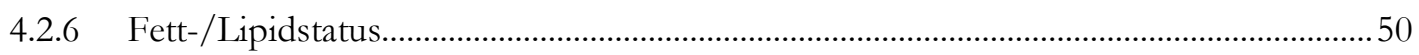

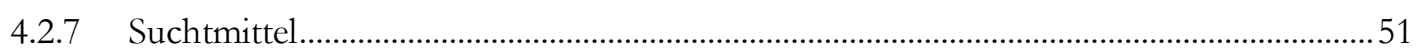

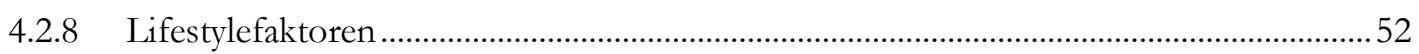

4.3 Kurzzeit-Follow-up der lean-NAFLD im Vergleich zur non-lean-NAFLD..................53

4.4 Effekte einer standardisierten Kurzberatung im Kurzzeitintervall ...............................54

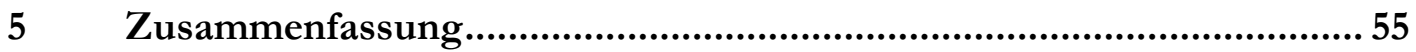

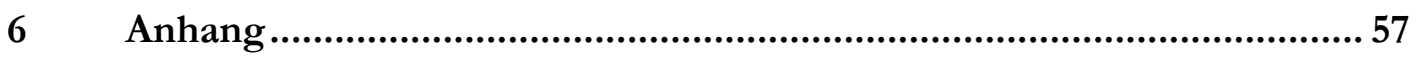

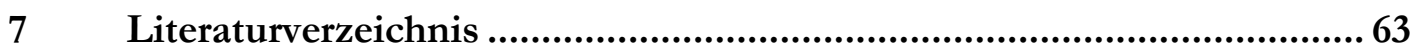




\section{Abbildungsverzeichnis}

Abbildung 1: Pathophysiologie der NAFLD ............................................................................... 1

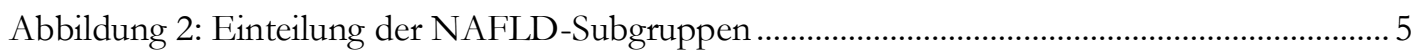

Abbildung 3: Diagnostischer Pfad im LZG ............................................................................ 15

Abbildung 4: Studienkollektiv und Subgruppierungen............................................................2 21

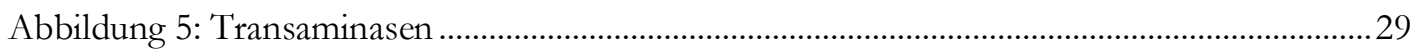

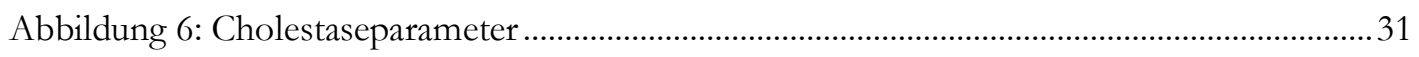

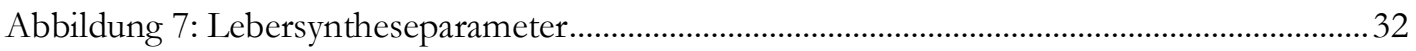

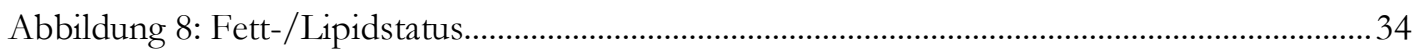

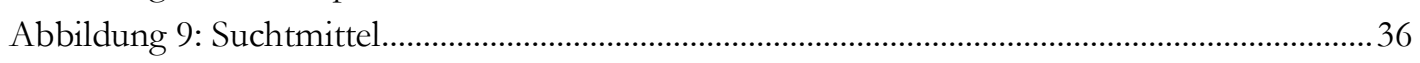

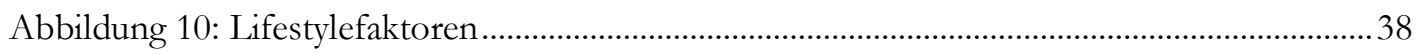

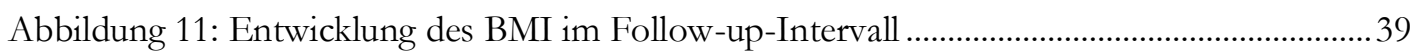

Abbildung 12: Absolute Veränderung ausgewählter Laborparameter im Follow-up-Intervall .43

Abbildung 13: "Gewichtsverlust" vs. "kein Gewichtsverlust" ....................................................4 44 


\section{Tabellenverzeichnis}

Tabelle 1: Häufigste sekundäre Ursachen einer Steatosis Hepatis................................................. 4

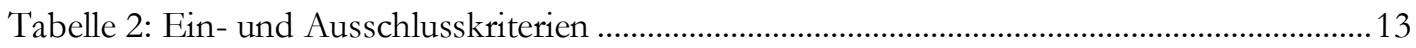

Tabelle 3: Laborchemische Referenzbereiche................................................................................ 18

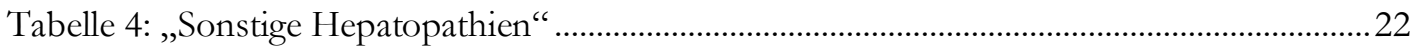

Tabelle 5: Epidemiologische Charakteristika des Kollektivs ........................................................2 24

Tabelle 6: Anthropometrische Charakteristika des Kollektivs .....................................................25

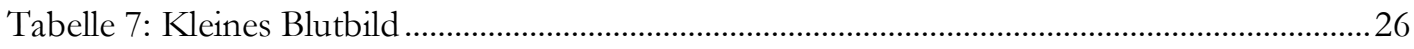

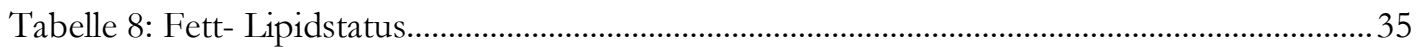

Tabelle 9: Absolute Veränderung ausgewählter Parameter im Follow-up-Intervall.................... 40

Tabelle 10: „Gewichtsverlust“ vs. ,kein Gewichtsverlust““ ............................................................46 


\section{Abkürzungsverzeichnis}

AFLD

$\mathrm{AIH}$

ALT

AMA

ANA

ANCA

AP

AST

BMI

CDT

CLD

DHC

DM II

DOAK

EDTA

EK

EtG

EtS

gGT

HBV

$\mathrm{HCV}$

HDL

HELLP

HFE

$\operatorname{Ig} G$

$\operatorname{IgM}$

INR

LAL

LC-1

LDL

Li-Heparin

LKM

LTx

LZG

MAD

MCV alcoholic fatty liver disease, alkoholische Fettlebererkrankung

Autoimmunhepatitis

Alanin-Aminotransferase

Antimitochondriale Antikörper

Antinukleäre Antikörper

anti neutrophil cytoplasmic antibodies, anti-neutrophile zytoplasmatische Antikörper

Alkalische Phosphatase

Aspartat-Aminotransferase

body mass index

carbohydrate-deficient transferrin, kohlenhydrat-defizientes Transferrin bzw. Desialotransferrin

chronic liver disease, chronische Lebererkrankungen

Ductus hepaticus communis

Diabetes mellitus, Typ 2

Direkte orale Antikoagulantien

Ethylendiamintetraessigsäure

Erstkontakt

Ethylglucuronid

Ethylsulfat

Gamma-Glutamyltranspeptidase

Hepatitis-B-Virus

Hepatitis-C-Virus

high density lipoprotein, $\alpha$-Lipoprotein

haemolysis, elevated liver ensymes, low platelet count, hämolytische Anämie, erhöhte Leberenzyme, Verminderung der Thrombozytenzahl

buman hemochromatosis gene, humanes Hämochromatose-Gen

Immunglobulin G

Immunglobulin $\mathrm{M}$

international normalized ratio

bysosomal acid lipase, lysosomale saure Lipase

liver cytosolic protein antibodies, Leber-Zytosolprotein-Antikörper

bigh density lipoprotein, $\beta$-Lipoprotein

Lithium-Heparin

liver kidney microsomal antibodies, Antikörper gegen Leber- und

Nierenmikrosomen

Lebertransplantationen

Leberzentrum Göttingen

Mittlerer arterieller Druck

mean corpuscular volume, mittleres korpuskuläres Volumen 
NAFLD non-alcobolic fatty liver disease, nichtalkoholische Fettlebererkrankung

$\mathrm{NASH}$ non-alcoholic steatohepatits, nichtalkoholische Steatohepatitis

PBC Primär billäre Cholangitis

PCR polymerase chain reaction, Polymerase-Kettenreaktion

PSC Primär sklerosierende Cholangitis

py pack year, Packungsjahr

RR

Nichtinvasive Blutdruckmessung nach Riva-Rocci

SLA soluble liver antigen antibodies, Antikörper gegen lösliches Leberantigen

SMA smooth muscle antibodies, Antikörper gegen die glatte Muskulatur

RKI Robert Koch-Institut

UMG Universitätsmedizin Göttingen

WHO World Health Organization

WMA World Medical Association, Weltärztebund

WV Wiedervorstellung 


\section{$1 \quad$ Einleitung}

Die nichtalkoholische Fettlebererkrankung bzw. non-alcoholic fatty liver disease (NAFLD) gilt als die hepatische Manifestation des metabolischen Syndroms (Roeb et al. 2015) und hat sich, aufgrund des westlichen Lebensstils, zu einer der führenden Hepatopathien (Weiss et al. 2014; Roeb et al. 2015; Schwimmer et al. 2006), Ursache chronischer Lebererkrankungen (chronic liver disease, CLD) (Caldwell et al. 1999) und zunehmender Indikation für Lebertransplantationen (LTx) entwickelt (Agopian et al. 2012; Zezos und Renner 2014; Roeb et al. 2015; Holmer et al. 2018).

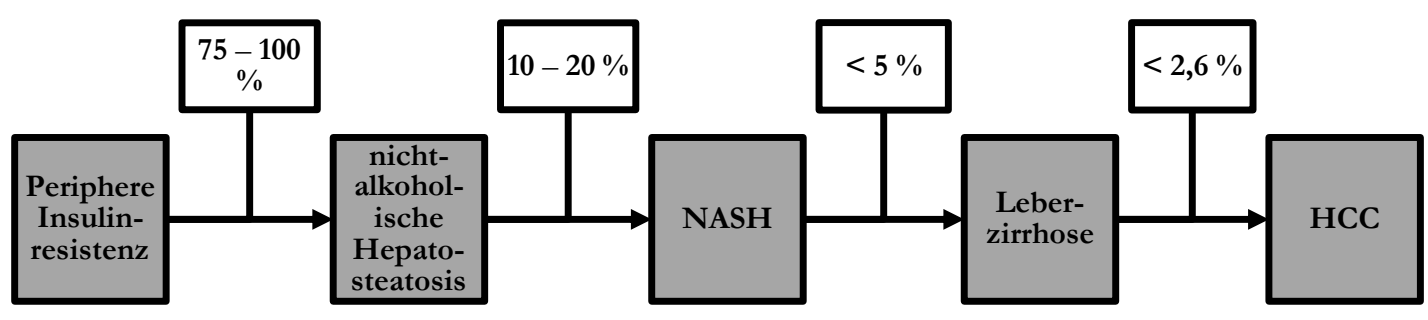

\section{Relatives HCC-Risiko}

Abbildung 1: Pathophysiologie der NAFLD Die Grafik zeigt die möglichen Manifestationsstadien der NAFLD im Verlauf bzw. die beobachteten Progressionsraten. Das HCC-Risiko ist bereits im Stadium der peripheren Insulinresistenz signifikant erhöht und steigt mit zunehmender Progression der NAFLD kontinuierlich an.

(nach Kawada et al. 2009; Denzer et al. 2009; Marmur et al. 2010; Cusi 2012; Greten et al. 2013; Weiss et al. 2014; Roeb et al. 2015; D'Avola et al. 2016; Estes et al. 2017)

HCC: hepatocellular carcinoma, NAFLD: non-alcoholic fatty liver disease, NASH: non-alcoholic steatohepatitis

Das Akronym NAFLD umfasst im Allgemeinen sämtliche Fettleber-Hepatopathien, die nicht mit Alkohol assoziiert bzw. im engeren Sinne sekundär, also durch andere Hepatopathien, erklärt werden können. Unter den Obergriff fallen sowohl die nichtzirrhotischen bzw. -entzündlichen Hepatosteatosen als auch die progredienten Verlaufsformen (siehe Abbildung 1: Pathophysiologie der NAFLD). Die Erstmanifestation stellt die klinisch inapparente Hepatosteatosis dar, der je nach Studie in ca. $75-100 \%$ eine periphere Insulinresistenz vorangeht (Weiss et al. 2014). In ca. 10 - 20\% der Fälle entwickelt sich im 
Verlauf eine Steatohepatitis (non-alcoholic steatohepatits, NASH) mit nur noch bedingtem Regressionspotential (Roeb et al. 2015). Eine Fibrose/Zirrhose des Parenchyms wird in bis zu $5 \%$ dieser Fälle erwartet (Cusi 2012). Die Entwicklung eines (transplantationspflichtigen) hepatozellulären Karzinoms (bepatocellular carcinoma, HCC) kann aktuell nicht sicher prognostiziert werden. In klinischen Studien wird die jährliche Inzidenz jedoch mit bis zu 2,6 \% bei vorliegender NASH angegeben (Ascha et al. 2010). Aktuell ist die NAFLD bereits für bis zu $22 \%$ der HCC-Fälle verantwortlich (D'Avola et al. 2016). Eine klinisch wichtige Besonderheit besteht darin, dass ähnlich zur Hepatitis-B-Infektion ein HCC auch ohne Vorliegen einer Leberzirrhose vermehrt entsteht (Greten et al. 2013). Zusätzlich muss konstatiert werden, dass die NASH als Indikation für Lebertransplantationen (LTx) stark zugenommen hat (Roeb et al. 2015). Bereits zum heutigen Zeitpunkt stellt sie die zweithäufigste Indikation einer LTx in den Vereinigen Staaten dar (Wong et al. 2014; Wong et al. 2015). Im Zeitraum 2002 - 2014 stieg ihr Anteil um mehr als das Siebenfache auf 16,2 \% bei US-amerikanischen LTx-Kandidaten.

\subsection{Definition}

Die Diagnose einer NAFLD erfordert nach aktuellem Stand drei Kernkriterien: eine Steatosis von min. $5 \%$ des Leberparenchyms, den Ausschluss einer alkoholassoziierten Steatosis, d. h. alcoholic fatty liver disease (AFLD) sowie den Ausschluss einer sekundären Hepatosteatosis (Zein et al. 2011; Weiss et al. 2014; Roeb et al. 2015).

\subsubsection{Steatosis}

Im Allgemeinen wird die ,histologische Anreicherung von Lipiden im Zytoplasma der Hepatozyten“ von mindestens $5 \%$ des Parenchyms als untere Nachweisgrenze gefordert (Roeb et al. 2015). Je nach Ausmaß des histologischen Verfettungsgrades kann eine Unterteilung in milde bzw. geringgradige $(<33 \%)$, mäßige bzw. mittelgradige $(33-66 \%)$ sowie eine schwere bzw. hochgradige Steatosis $(>66 \%$ ) vorgenommen werden (Tannapfel et al. 2010; Roeb et al. 2015), aus der sich z. T. Aussagen über das Progressions- bzw. Regressionspotential treffen lassen (Lallukka et al. 2017). Weitere feinhistologische Aufarbeitungen sind darüber hinaus zur Differentialdiagnose AFLD möglich, jedoch oft nicht eindeutig (Tannapfel et al. 2011; Yeh und Brunt 2014). Insgesamt wird die Forderung nach einer Biopsie zur Diagnosesicherung aufgrund zahlreicher Limitationen ökonomischer und organisatorischer Natur bzw. einer heterogenen Risiko-Nutzen-Bewertung zunehmend seltener gestellt (Buzzetti et al. 2015; Thavorn und Coyle 2015; Roeb et al. 2015; Wong et al. 2017). Das aktuelle Standardverfahren zur Detektion einer Fettleber ist die transkutane Sonografie. Mit dieser ist die zuverlässige Detektion einer hepatischen Steatosis bei mittlerer bzw. höhergradiger Verfettung mit einer Sensitivität von bis zu 85 \% bzw. Spezifität von bis 
zu $94 \%$ möglich. In Abhängigkeit der Untersucherexpertise und des verwendeten Gerätes können diese bei niedrigen Steatosegraden geringer ausfallen (Roeb et al. 2015; Bohte et al. 2011).

\subsubsection{Ausschluss einer AFLD}

Als wichtigste Differentialdiagnose muss ein „pathologischer“ Alkoholkonsum als SteatosisUrsache ausgeschlossen werden (Reid 2001; Roeb et al. 2015). International konnte bis zum heutigen Tag kein einheitlicher Konsens über einen Ethanol-Grenzwert für die tägliche oder wöchentliche perorale Aufnahme getroffen werden.

Im deutschsprachigen Raum gilt vielfach ein täglicher Konsum von $\geq 30 \mathrm{~g} / \mathrm{d}$ (Männer) bzw. $\geq 20 \mathrm{~g} / \mathrm{d}$ (Frauen) als nicht vereinbar mit der Diagnose einer NAFLD (Weiss et al. 2014). Die deutsche S2k-Leitlinie „Nicht-alkoholische Fettlebererkrankungen“ empfiehlt abweichend hierzu bereits eine Schwellenwertdosis von $\geq 20 \mathrm{~g} / \mathrm{d}$ (Männer) bzw. $\geq 10 \mathrm{~g} / \mathrm{d}$ (Frauen) (Roeb et al. 2015).

Grundsätzlich ist der eindeutige Nachweis eines chronischen, pathologischen Alkoholkonsums bei fehlender Kooperation des Patienten i. d. R. schwierig. Im klinischen Alltag erfolgt diese Differentialdiagnose v. a. über (fremd)-anamnestische Daten bzw. laborchemische Hinweise (u. a. isolierte gGT- bzw. MCV-Erhöhungen). Eine „objektivierte“ Erhebung des Alkoholkonsums z. B. mittels carbohydrate deficient transferrin (CDT), Ethylglucuronid (EtG) bzw. Ethylsulfat (EtS) Bestimmung, erfolgt aus ökonomischen und organisatorischen Gesichtspunkten häufig nicht. In Zweifelsfällen kann die feinhistologische Begutachtung den Verdacht auf eine AFLD zusätzlich erhärten (Tannapfel et al. 2010; Roeb et al. 2015). Häufig gelingt die Differentialdiagnose erst über Monate und Jahre der regelmäßigen Kontrolle und eine (oft erschwerte bzw. nicht mögliche) Fremdanamnese. 


\subsubsection{Sekundäre Steatosen}

Obligat vor Diagnosestellung der NAFLD ist der Ausschluss einer sekundären Steatosis (siehe Tabelle 1):

Tabelle 1: Häufigste sekundäre Ursachen einer Steatosis Hepatis

\begin{tabular}{|c|c|}
\hline \multirow{2}{*}{ Virale Hepatitiden } & Hepatitis-B-Virus \\
\hline & Hepatitis-C-Virus \\
\hline \multicolumn{2}{|l|}{ Morbus Wilson } \\
\hline \multirow{2}{*}{ Schwangerschaft } & Akute Schwangerschaftsfettleber \\
\hline & HELLP-Syndrom \\
\hline \multirow{7}{*}{ Stoffwechselstörungen } & Hypo-/Abetalipoproteinäme \\
\hline & Familiäre Hyperlipidämie \\
\hline & Glykogenosen \\
\hline & Weber-Christian-Syndrom \\
\hline & Lipodystrophie \\
\hline & Hereditäre Fruktoseintoleranz \\
\hline & $\alpha 1$-Antitrypsin-Mangel \\
\hline \multirow{4}{*}{ Medikamente } & Zytostatika \\
\hline & Aspirin \\
\hline & Amiodaron \\
\hline & etc. \\
\hline \multirow{3}{*}{$\begin{array}{l}\text { Ernährungsbedingte } \\
\text { Ursachen }\end{array}$} & Kurzdarmsyndrom \\
\hline & Mangelernährung \\
\hline & Totale parenterale Ernährung \\
\hline \multicolumn{2}{|l|}{ Reye-Syndrom } \\
\hline \multirow{2}{*}{ Darmerkrankungen } & Chronisch entzündliche Darmerkrankungen \\
\hline & Zöliakie \\
\hline
\end{tabular}

(nach Weiss 2014; Vespasiani-Gentilucci 2014; Roeb 2015; Robinson 2012)

HELLP: haemolysis, elevated liver enฐymes, low platelet count, hämolytische Anämie, erhöhte Leberenzyme, Verminderung der Thrombozytenzahl 


\subsection{Sonderform: lean-NAFLD}

Lange Zeit wurde die NAFLD vor dem Hintergrund ihrer Risikofaktoren v. a. mit Faktoren des metabolischen Syndroms assoziiert. Seit ca. zehn Jahren rückt zunehmend die Gruppe normalgewichtiger NAFLD-Patienten in den klinischen und wissenschaftlichen Fokus. Diese Konstellation wird als sog. „lean-NAFLD“ beschrieben und findet sich je nach Studie bei 7 - $19 \%$ aller NAFLD-Patienten bzw. bis zu $9 \%$ der normalgewichtigen Bevölkerung (Cohen et al. 2011; Younossi et al. 2012; Margariti et al. 2013; Akyuz et al. 2015; Yoshitaka et al. 2017; Kumar und Mohan 2017; Hagström et al. 2018). Explizit sei an dieser Stelle auf den asiatischen Raum hingewiesen, in dem der Anteil der lean-NAFLD an der Gesamtzahl der NAFLD-Fälle zwischen $30 \%$ (Korea) und > $70 \%$ (Indien) beträgt (Younossi et al. 2018a) bzw. bei bis zu jedem 5. gesunden Normalgewichtigen vorliegt (Wei et al. 2015).

Die Weltgesundheitsorganisation (World Health Organization, WHO) hat zur Einordnung des Ernährungszustandes in mitteleuropäischen Kollektiven folgende BMI-Grenzwerte festgelegt: Untergewicht $<18.50 \mathrm{~kg} / \mathrm{m}^{2}$, Normalgewicht $18.50-24.99 \mathrm{~kg} / \mathrm{m}^{2}$, Übergewicht/Präadipositas 25.00 - $29.99 \mathrm{~kg} / \mathrm{m}^{2}$, Adipositas $\geq 30.00 \mathrm{~kg} / \mathrm{m}^{2}$ (World Health Organization 2000). Diese wurden in den letzten Jahren zunehmend auf die Definition der NAFLD-Subgruppen übertragen (Wattacheril und Sanyal 2016; Fracanzani et al. 2017; Feldman et al. 2017; Sookoian und Pirola 2017; Hagström et al. 2018) (siehe Abbildung 2).

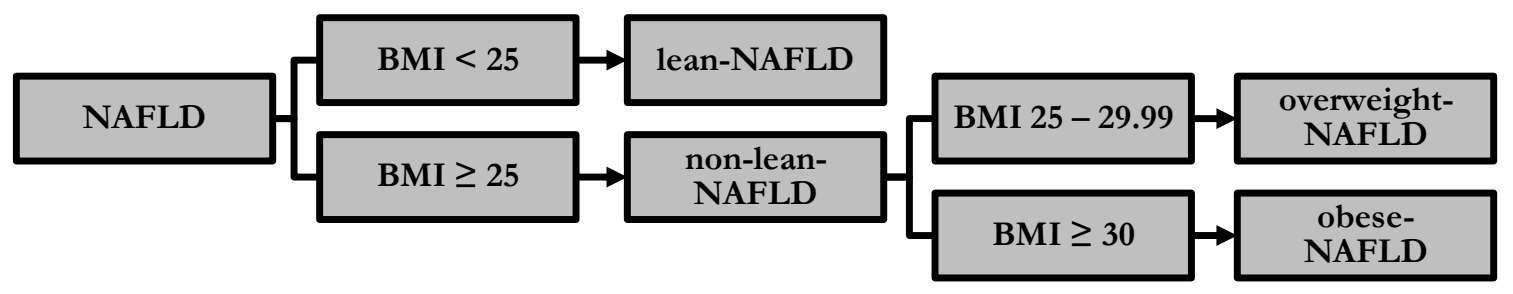

Abbildung 2: Einteilung der NAFLD-Subgruppen Für diese Studie verwendete Einteilung eines NAFLDKollektivs nach den WHO-BMI-Grenzwerten.

(nach World Health Organization 2000; Hagström et al. 2018; Wattacheril und Sanyal 2016; Fracanzani et al. 2017; Feldman et al. 2017; Sookoian und Pirola 2017)

BMI: body mass index, NAFLD: non-alcoholic fatty liver disease, WHO: World Health Organization

Eine jüngst erschienene schwedische Arbeit untersuchte erstmalig die NAFLD nach o.g. Schema in einer europäischen Studienpopulation im Langzeit-Follow-up. Nach 20 Jahren des Follow-ups ließ sich zwar keine erhöhte Gesamtmortalität, jedoch ein deutlich erhöhtes Risiko für schwere Lebererkrankungen bei lean-NAFLD-Patienten nachweisen (Hagström et al. 2018). Inwieweit die lean-NAFLD somit eine relevante Sonderform in ätiologischer, pathophysiologischer und ggf. therapeutischer Hinsicht darstellt, ist zum derzeitigen Zeitpunkt Gegenstand intensiver Forschung. Zudem ist unklar, inwieweit die lean-NAFLD eine hepatische Frühmanifestation eines späteren metabolischen Syndroms ist, eine 
eigenständige Entität darstellt bzw. bezüglich des Risikoprofils der NAFLD (u. a. des HCCRisikos) entspricht. Insbesondere in Bezug auf mögliche therapeutische Angriffspunkte besteht derzeit noch erheblicher Forschungsbedarf.

\subsection{Epidemiologie}

Die weltweite NAFLD-Prävalenz wird u. a. aufgrund unterschiedlicher Diagnosekriterien kontrovers angegeben. Laut Vernon et al. geht ein Großteil an Studien von einer weltweiten Prävalenz zwischen $6-33 \%$ aus (Vernon et al. 2011), während eine Studie an gesunden, potentiellen Leberlebendspendern sogar eine Prävalenz von $>50 \%$ bei jungen Erwachsenen beobachtete (Lee et al. 2007). In entwickelten Industriestaaten liegen die Schätzungen für die NAFLD-Prävalenz insgesamt bei einem Drittel der Gesamtbevölkerung (Zhou et al. 2012; Lazo et al. 2013; Koehler et al. 2013; Blachier et al. 2013). Studien an mitteleuropäischen Studienpopulationen beschreiben übereinstimmend Prävalenzen von 30,4 \% (Blachier et al. 2013) bis 34,9 \% (Koehler et al. 2013; Younossi et al. 2018a).

Unstrittig ist die steigende Prävalenz der NAFLD (Alexander et al. 2018). Zwischen 1988 und 2010 konnte eine Vervierfachung der Prävalenz (Welsh et al. 2013), bzw. ein Zuwachs der Patientenzahlen mit NASH-Zirrhose um $250 \%$ (Kabbany et al. 2017) festgestellt werden. Für die nähere Zukunft wird zwischen 2015 und 2030 eine weitere Zunahme der NAFLDFälle um $21 \%$, der NASH um $63 \%$, der dekompensierten NASH-Leberzirrhosen um $168 \%$, der NASH-assoziierten HCC-Fälle um $137 \%$ und der dadurch bedingten hepatischassoziierten Todesfälle um $178 \%$ erwartet (Estes et al. 2017).

Aktuell hat sich die NAFLD bereits zur häufigsten chronischen Lebererkrankung (CLD) im Kindes- und Erwachsenenalter entwickelt (Weiss et al. 2014; Roeb et al. 2015). Die NAFLD stellt bereits heute die häufigste Leberabnormalität des Kindes-/Jugendalters dar und liegt bei bis zu $10 \%$ aller Kinder vor (Schwimmer et al. 2006). Liegt komorbid eine Adipositas vor, so steigt die Prävalenz in entsprechenden Kohorten auf bis zu $67 \%$ aller Kinder (Denzer et al. 2009; Eng et al. 2014). Liegt ein metabolisches Syndroms vor, steigt das Risiko einer NAFLD um bis zu 300 \% (Papandreou et al. 2017). Aktuell weisen bereits bis zu 4.000 Kinder in Deutschland ein „erhöhtes Risiko für eine frühzeitig progrediente Leberfibrose bzw. Zirrhose“, aufgrund einer NAFLD auf (Roeb et al. 2015). 


\section{4 Ätiologie}

\subsubsection{Metabolisches Syndrom}

Die besten Studienergebnisse liegen derzeit für die starke Assoziation zwischen NAFLD und metabolischem Syndrom (gestörte Insulinsensitivität bzw. Diabetes mellitus Typ 2 (DM II), Adipositas, arterielle Hypertonie bzw. Dyslipoproteinämie und Hypertriglyceridämie) vor (Roeb et al. 2015; Caldwell et al. 1999; Rajindrajith et al. 2017). Ab dem Vorliegen eines der o. g. Kriterien, besteht ein signifikant erhöhtes Risiko für eine NAFLD; liegen mehrere Kriterien vor, potenziert sich dieses (Rajindrajith et al. 2017). Eine Erhöhung des Hüftumfangs verfünffacht die Wahrscheinlichkeit einer NAFLD (Leite et al. 2009). Zusätzlich wird durch ein metabolisches Syndrom das Schwereausmaß bzw. die frühe Tendenz zur Progredienz deutlich begünstigt (Denzer et al. 2009). Insoweit lässt sich die ausgeprägte Zunahme der NAFLD mit einem starken Anstieg der Prävalenz des metabolischen Syndroms assoziieren (Alberti et al. 2009; Mensink et al. 2013; OECD 2016; Tamayo et al. 2016). V. a. Kinder sind durch die Zunahme des metabolischen Syndroms bzw. Übergewichts (ca. $22 \%$ ) oder DM II (bis zu $10 \%$ ) durch eine frühzeitige und akzelerierte hepatische Progredienz bedroht (Schwimmer et al. 2006; Kurth und Schaffrath Rosario 2007; Feldstein et al. 2009; OECD 2016).

\subsubsection{Suchtmittel/Lifestyle}

Die Bedeutung eines „gemäßigten“ Alkoholkonsums wird kontrovers bewertet. Die derzeitigen Leitlinienempfehlungen sprechen sich aus präventiver Sicht für eine deutliche Reduktion bzw. absolute Karenz von Alkohol aus (Roeb et al. 2015). Lediglich wenige Studien diskutieren mögliche protektive Effekte z. B. im Sinne niedrigerer Fibrosestadien, durch einen gemäßigten Alkoholkonsum in NAFLD-Kollektiven (Jayasekara et al. 2014; Dunn et al. 2012; Hagström et al. 2017). Die Bedeutung dieser Ergebnisse ist derzeit strittig. Tabakabusus, aktiv wie auch passiv wird eindeutig mit einer NAFLD assoziiert (Okamoto et al. 2018). Insbesondere Patienten mit frühem Expositionsbeginn (Kindes- und Jugendalter) und komorbidem Übergewicht weisen eine Potenzierung des NAFLD-Risikos um das Achtfache auf (Liu et al. 2013). Zusätzlich belegt ist eine direkte Korrelation zwischen Rauchbelastung und Schweregrad der Steatose bzw. histologischen Fibrose (Zein et al. 2011).

Bezüglich diverser Diätformen liegen derzeit heterogene Studienergebnisse vor. Der erhöhte Softdrink-Konsum wird allgemein als ätiologisch relevant angesehen (Assy et al. 2008; Abid et al. 2009; Malik et al. 2010; Hashemi Kani et al. 2014). Gleiche Effekte scheinen ebenso bei stark kohlenhydrat-/fruktosereichen Diätformen (Sathiaraj et al. 2011; Nobili et al. 2017) bzw. häufigen Zwischenmahlzeiten (sog. ,snacking") feststellbar zu sein (Koopman et al. 2014). Der Einfluss proteinarmer Ernährungsformen ist aktuell unklar und Gegenstand weiterer Forschung (Sathiaraj et al. 2011; Hashemi Kani et al. 2014). 
Eine weitere, ausgeprägte Assoziation liegt zwischen NAFLD und allgemeinem Mangel an physischer Aktivität vor (Zelber-Sagi et al. 2008; Kistler et al. 2011; Bae et al. 2012; Oddy et al. 2013), welche insgesamt den Großteil der westlichen Bevölkerung betrifft (OECD 2016).

Die Auswirkungen eines regelmäßigen Kaffeekonsums werden mit Ausnahme weniger Studien (Shen et al. 2016; Veronese et al. 2018) insgesamt als präventiv günstig bzw. progressionshemmend eingeschätzt (Birerdinc et al. 2012; Yesil und Yilmaz 2013; Marventano et al. 2016; Gupta et al. 2015; Molloy et al. 2012). Insbesondere Effekte auf die Fibrose- und Zirrhoseneigung (Klatsky et al. 2006; Anty et al. 2012) werden, bei aktuell unklarem pathophysiologischem Mechanismus, diskutiert (Yesil und Yilmaz 2013; Bae et al. 2017). Zusätzliche Wechselwirkungen mit der Entstehung bzw. des Verlaufs einer peripheren Insulinresistenz (Bambha et al. 2014; Gupta et al. 2015), Mechanismen intrahepatischer Geninduktion (Kalthoff et al. 2010), antioxidativer Effekte (GutiérrezGrobe et al. 2012) sowie einer gesteigerten Autophagie intrahepatischen Fettes werden in diesem Kontext diskutiert (Sinha et al. 2014).

\subsection{Komplikationen}

Klinische Bedeutung erhält die NAFLD häufig v. a. aufgrund ihrer fortgeschrittenen Stadien (NASH, Zirrhose bzw. HCC). Negative Auswirkungen auf die Lebensqualität lassen sich indessen früher, z. T. bereits bei klinisch asymptomatischen Hepatosteatosen feststellen (Golabi et al. 2016; Kennedy-Martin et al. 2017; Assimakopoulos et al. 2018). Unabhängig von hepatischen Komplikationen gehen bereits (asymptomatische) Hepatosteatosen mit einer signifikant erhöhten Wahrscheinlichkeit eines metabolisches Syndroms (Ryoo et al. 2013) bzw. eines DM II (Bae et al. 2011; Choi et al. 2013; Zelber-Sagi et al. 2013; Bae et al. 2018), symptomatischer Gallensteine (Chen et al. 2014), eines kolorektalen Karzinoms (Stadlmayr et al. 2011; Lee et al. 2012; Shen et al. 2014; Kim et al. 2017), kardiovaskulärer Komplikationen (Ekstedt et al. 2006; Vlachopoulos et al. 2010; Wong et al. 2011; Stepanova und Younossi 2012; Kozakova et al. 2012; Kim et al. 2012; Roeb et al. 2015; Abdeldyem et al. 2017; Lee et al. 2017), chronischer Niereninsuffizienzen (Mantovani et al. 2017; Wijarnpreecha et al. 2017) sowie einem schlechteren Outcome im Rahmen leberchirugischer Eingriffe inkl. Transplantationen (Gabrielli et al. 2012; Reddy et al. 2012) einher. V. a. Frauen scheinen im direkten Vergleich mit Patienten ohne NAFLD von einer deutlichen Mortalitätssteigerung betroffen zu sein (Hwang et al. 2017).

Obwohl das Risiko der NAFLD-Progredienz $\mathrm{zu}$ hochgradigen chronischen Lebererkrankungen für die Einzelperson (vergleichsweise) gering ist, ergibt sich aufgrund der hohen Prävalenz in der Gesamtbevölkerung eine große Anzahl direkt Betroffener und eine Langzeitmortalität von bis zu 83,3 \% in fortgeschrittenen NAFLD-Stadien (Hashimoto und Tokushige 2012). Abgesehen von unklaren Auswirkungen einer NAFLD, z. B. auf die Arzneimittelmetabolisierung (Merrell und Cherrington 2011; Roeb et al. 2015), sollen im Folgenden relevante Komplikationen kurz beleuchtet werden: 


\subsubsection{Leberzirrhose}

Explizit erwähnt werden muss die Leberzirrhose als mögliche NAFLD-Komplikation, die bei bis zu $5 \%$ aller NASH-Fälle zu erwarten ist (Roeb et al. 2015). Mittlerweile konnte gezeigt werden, dass die NASH die Hauptursache für bis dahin als „kryptogen“ klassifizierte Leberzirrhosen darstellt (Caldwell et al. 1999; Marmur et al. 2010). Die „kryptogene“ Leberzirrhose stellt zudem nicht selten die klinische Erstmanifestation dar (Wisocky und Paul 2017). Zirrhosetypische Komplikationen (portale Hypertension mit Ausbildung von Umgehungskreisläufen, Lebersynthesestörungen, Blutungskomplikationen, hepatische Enzephalopathie, Aszites, etc.) und v. a. auch die HCC-Zunahme (Hashimoto und Tokushige 2012) sind zusätzlich zu erwarten. Eine kürzlich publizierte Studie weist in diesem Kontext auf möglicherweise schwerwiegende Verläufe in lean-Kollektiven hin (Hagström et al. 2018).

\subsubsection{Hepatozelluläres Karzinom}

Das hepatozelluläre Karzinom (HCC) stellt eine weitere Stufe der Progression dar. Bereits Übergewicht, eine periphere Insulinresistenz oder ein Diabetes mellitus stellen unabhängige Risikofaktoren für die Entwicklung eines HCC dar (Margini und Dufour 2016; El-Serag et al. 2004; Kim et al. 2017), während sich dieses Risiko bei Hepatosteatosis nochmals deutlich erhöht (Kawada et al. 2009; Greten et al. 2013) und bei NASH-Zirrhose bei bis zu 2,5 \% (1-Jahres-Inzidenz) bzw. 12,8 \% (3-Jahres-Inzidenz) liegt (Ascha et al. 2010; White et al. 2012; Kanwal et al. 2018). Zurzeit liegt die NASH an zweiter Stelle hinter den Virushepatitiden als Ursache eines HCC (Ertle et al. 2011), während das HCC gleichzeitig die häufigste Todesursache in diesen Fällen darstellt (Yatsuji et al. 2009). Erkenntnisse der letzten Jahre zeigen eine Zunahme des Anteils der NASH-assoziierten HCC-Fälle um mehr als $700 \%$ über einen 14-Jahres-Zeitraum (Younossi et al. 2018b), sodass das Aufrücken der NAFLD/NASH als Hauptursache des HCC in entwickelten Industriestaaten in den nächsten Jahren erwartet wird.

\subsubsection{Notwendigkeit einer Lebertransplantation}

Neben einem HCC stellt ein Leberversagen mit Transplantationspflichtigkeit einen weiteren Maximalverlauf dar. Liegt eine NAFLD im Kindesalter vor, steigt das Risiko in den nächsten 20 Jahren eine Lebertransplantation zu benötigen deutlich an (Feldstein et al. 2009). In den USA ist die NAFLD bzw. NASH-Zirrhose bereits heute die zweithäufigste Indikation für eine LTx (Wong et al. 2014; Wong et al. 2015; Roeb et al. 2015), welche ihrerseits durch ein insgesamt schlechtes Outcome, auf dem Niveau einer äthyltoxischen bzw. virusassoziierten Transplantationsindikation, gekennzeichnet ist (Malik et al. 2009; Reddy et al. 2012; Vanwagner et al. 2012; Cauchy et al. 2013; Wang et al. 2014; Agopian et al. 2012). 


\subsection{Kosten/ökonomische Faktoren}

Die klinische und ökonomische Bedeutung der NAFLD wurde bzw. wird aktuell noch häufig unterschätzt (Wieland et al. 2013; Blais et al. 2015; Alexander et al. 2018; Sanyal 2018). In den letzten Jahren wächst v. a. das ökonomische Problembewusstsein stark an: Bereits eine Hepatosteatosis in Verbindung mit Transaminasenerhöhung führt zu einem Kostenplus von bis zu $26 \%$ in der Betreuung betroffener Patienten (Baumeister et al. 2008; Ghamar et al. 2013; Zhang et al. 2015). Während die NAFLD-assoziierten Kosten im Vereinigten Königreich innerhalb eines 5-Jahreszeitraums um mehr als 50\% anstiegen (Younossi et al. 2015), liegen die direkten medizinischen Kosten in den USA aktuell bei ca. 103 Milliarden \$/Jahr für 64 Millionen, bzw. 35 Milliarden $€$ für 52 Millionen Betroffene in den Staaten Deutschland, Frankreich, Italien und dem Vereinigten Königreich (Younossi et al. 2016).

Der Gesamtkostenfaktor der chronischen Lebererkrankungen, deren zunehmender bzw. überwiegender Teil in naher Zukunft mutmaßlich auf eine NAFLD zurückzuführen sein wird, wird aktuell ein Gesamtkostenfaktor von umgerechnet bis zu 41 Milliarden $€ / J a h r$ für das australische Gesundheitssystem zugeschrieben. Dieser Betrag liegt schon heute deutlich über den Kosten des Diabetes mellitus (umgerechnet 28 Milliarden $€ / J a h r$ ) (Gastroenterological Society of Australia 2013). Nicht zuletzt sozioökonomische Kosten steigen durch CLD massiv an (Stepanova et al. 2017) und führen zu einer zunehmenden Belastung der Sozialsysteme weltweit.

Unterstrichen werden diese Anhaltspunkte durch die Einschätzungen von Wirtschaftsanalysten, die den aktuellen Marktwert zukünftiger NASH-Medikamente mit 8 - 40 Milliarden \$/Jahr allein für den US-amerikanischen Markt beziffern (Crow 2014) und zu Milliardeninvestitionen der Pharmaindustrie führen (Gatlin 2016).

Vor diesem Hintergrund wird zunehmend ein Bevölkerungsscreening diskutiert. Obwohl eine zeitnahe Intervention ökonomisch sinnvoll erscheint (Tanajewski et al. 2017), wird die Kosten-Nutzen-Analyse heterogen bewertet (Corey et al. 2016). Festzustehen scheint, dass ein positiver Effekt v. a. durch die nicht-invasive Diagnostik zu erwarten ist (Tapper et al. 2016; Zhang et al. 2015; Tapper et al. 2015; Crossan et al. 2015; Thavorn und Coyle 2015).

\subsection{Therapieansätze/Interventionen}

Bezüglich möglicher Therapieansätze und Interventionen besteht aus o.g. Anlass reges Forschungsinteresse. Insgesamt lässt sich festhalten, dass die Therapie nicht zuletzt aufgrund des geringen Effekts bisheriger medikamentöser Therapieregime (Roeb et al. 2015; Weiss et al. 2014; Byrne und Targher 2014) v. a. Lifestyle-assoziierte Maßnahmen (s. u.) berücksichtigt (Weiss et al. 2014; Byrne und Targher 2014; Roeb et al. 2015).

Die Reduktion des Körpergewichts stellt aktuell den therapeutischen Hauptansatzpunkt dar (Roeb et al. 2015; Weiss et al. 2014). Gewichtsverluste von 5 - 7 \% des Körpergewichts 
zeigten in Studien sowohl eine Verbesserung der laborchemischen Parameter (Ratziu et al. 2010; Thoma et al. 2012; Hohenester et al. 2018; Shidfar et al. 2018) als auch der histologischen Aktivität (Promrat et al. 2010; Chalasani et al. 2017). Je nach Studie werden Remissionsraten von bis zu $75 \%$ beschrieben (Wong et al. 2010; Zelber-Sagi et al. 2012), die sich z. T. bereits nach Kurzzeitintervention von $12-52$ Wochen einstellen (Utz-Melere et al. 2018; Hohenester et al. 2018; Shidfar et al. 2018).

Die Steigerung der physischen Aktivität stellt einen weiteren therapeutischen Grundpfeiler dar. Bereits nach wenigen Wochen treten signifikante Verbesserungen der Leberparameter ein (Utz-Melere et al. 2018; Molina-Molina et al. 2018). Insbesondere die Wahrscheinlichkeit einer Remission kann durch regelmäßiges Training deutlich erhöht werden (Osaka et al. 2018). Erzielen Patienten sowohl eine Gewichtsreduktion, als auch eine Zunahme der physischen Aktivität, wird zum gegenwärtigen Zeitpunkt der optimalste Effekt antizipiert (Chalasani et al. 2017; Zou et al. 2018; Shidfar et al. 2018; Utz-Melere et al. 2018).

Die Auswirkungen verschiedener Kostformen, u. a. der mediterranen Diät, auf das Regressionspotenzial einer NAFLD sind umstritten (Anania et al. 2018). Unterdessen werden positive Auswirkungen bezüglich der sonographischen Ausprägung der Leberverfettung, bzw. der Transaminasen zunehmend beschrieben (Ma et al. 2018; Katsagoni et al. 2018; Utz-Melere et al. 2018).

Einigkeit besteht bezüglich des ungünstigen Effekts eines erhöhten Softdrinkkonsums bzw. vermehrter Fruktoseaufnahme (Zelber-Sagi et al. 2007; Assy et al. 2008; Abid et al. 2009; Hashemi Kani et al. 2014; Asgari-Taee et al. 2018). Inwieweit eine Karenz/Reduktion bei bereits bestehender NAFLD zu einer Regression/Remission führt, ist derzeit nicht bekannt. 


\subsection{Fragestellungen}

\subsubsection{Differenzierbarkeit von AFLD und NAFLD anhand klinischer Routineparameter}

Die NAFLD entwickelt sich aktuell zur wichtigsten Differentialdiagnose der AFLD. Patienten mit äthyltoxischer Genese sind dem Arzt-Patienten-Gespräch häufig weniger gut zugänglich. Während die Etablierung von Spezial-Laborparametern (CDT, EtG, etc.) insgesamt bisher nicht vollständig gelungen ist, soll im ersten Schritt dieser Studie eine Reevaluation etablierter Routineparameter als Hilfsmittel zur Differentialdiagnose zwischen NAFLD und AFLD erfolgen.

\subsubsection{Kurzzeit-Follow-up der lean-NAFLD im Vergleich zur non-lean-NAFLD}

Im zweiten Arbeitsschritt wird das NAFLD-Kollektiv gesondert betrachtet und nach Subgruppierung (lean- vs. non-lean-NAFLD) anhand ausgewählter Routineparameter zwischen Erst- und Zweitkontakt verglichen. Es soll der Frage nachgegangen werden, ob ggf. eine Subgruppen-spezifische, unterschiedliche Entwicklung dieser Gruppen anhand etablierter Parameter im Kurzzeitintervall festzustellen ist.

\subsubsection{Effekte einer standardisierten Kurzberatung im Kurzzeitintervall}

Im letzten Schritt der Studie soll das Augenmerk auf das klinische und therapeutische Management gelegt werden. Aktuell beschränkt sich dies auf nichtmedikamentöse Maßnahmen im Sinne einer Lifestyleberatung zur moderaten Gewichtsreduktion, vermehrten körperlichen Aktivität und/oder Ernährungsumstellung. Diesbezüglich erfolgt bei Erstkontakt eine standardisierte Kurzberatung. Diese Arbeit soll sich im letzten Schritt der Frage widmen, ob und in welchem Maße diese Intervention im Kurzzeitintervall messbare Effekte zeigt. 


\section{Probanden und Methoden}

\subsection{Studienrahmen}

Im Rahmen dieser Untersuchung wurden über ein Jahr Daten von allen Patienten erhoben, die sich erstmalig im Leberzentrum Göttingen (LZG) der Universitätsmedizin Göttingen (UMG) vorstellten. Das LZG betreibt eine spezialisierte Hochschulambulanz mit über 2.000 Patientenkontakten pro Jahr und ist spezialisiert auf die Diagnostik und Therapie sämtlicher Lebererkrankungen und deren Komplikationen. Die Zuweisung der Patienten erfolgt i. d. R. durch niedergelassene Allgemeinmediziner bzw. Internisten oder klinikintern.

Die Patienten wurden im Jahr 2016 prospektiv in diese Analyse eingeschlossen und über einen Zeitraum von maximal einem Jahr nachbeobachtet. Die Einwilligung zur Datenverwendung war Voraussetzung hierzu. Alle Patienten erhielten vor Erstkontakt (EK) einen standardisierten Fragebogen (siehe Anlage). Die Patienten wurden im Anschluss von einem erfahrenen Hepatologen anamnestiziert und der Fragebogen vervollständigt. Es erfolgten Blutanalysen und eine Abdomensonografie. Nach Vorliegen dieser Ergebnisse konnte entweder eine Diagnose gestellt und ggf. eine Therapie eingeleitet werden oder es erfolgten im Verlauf weiterführende Untersuchungen (z. B. u. a. Biopsie).

Alle Patienten mit einer nichtalkoholischen Fettlebererkrankung wurden standardisiert (s. u.) hinsichtlich konservativer Therapiemaßnahmen beraten und hinsichtlich der Umsetzung und des klinischen und laborchemischen Verlaufs nachverfolgt.

\subsubsection{Ein- und Ausschlusskriterien}

Der Einschlusszeitraum erstreckte sich vom 05.01.2016 bis zum 31.12.2016 für Patienten mit Erstkontakt im LZG (siehe Abbildung 4). Die relevanten Ein- bzw. Ausschlusskriterien sind im Folgenden dargestellt (siehe Tabelle 2)

Tabelle 2: Ein- und Ausschlusskriterien

\section{Einschlusskriterien}

Volljährigkeit (18. Lebensjahr zum Zeitpunkt des Erstkontaktes)

Einverständniserklärung zur Datenverwendung für wissenschaftliche Zwecke

Erstvorstellung im LZG

Erhöhte Leberparameter bzw. Vorliegen einer klinischen/bildmorphologischen Leberläsion 


\section{Ausschlusskriterien}

Minderjährigkeit

Fehlende Einverständniserklärung, bzw. Ablehnung der Datenverwendung für wissenschaftliche Zwecke

Fehlende Aufklärungsfähigkeit (z. B. psychische Erkrankung oder sprachliche Barriere)

Fehlender Nachweis einer Lebererkrankung

Wiedervorstellung im LZG

Dargestellt sind die für diese Studie relevanten Ein- bzw. Ausschlusskriterien.

LZG: Leberzentrum Göttingen

\subsubsection{Datenschutz}

Sämtliche Unterlagen und Untersuchungsergebnisse unterliegen der ärztlichen Schweigepflicht und sind nur autorisiertem Personal des LZG zugänglich. Zur Wahrung des Datenschutzes wurden sämtliche Patientendaten pseudonymisiert auf Passwort-geschützten Computern im Universitätsklinikum Göttingen verarbeitet und dort in Passwort-geschützten Dateien gesichert. Eine Weitergabe an bzw. Verarbeitung durch externe Stellen oder Dritte erfolgte nicht.

\subsection{Datenerhebung}

Die Datenerhebung erfolgte standardisiert im Rahmen eines ärztlichen Anamnesegesprächs, klinischer Untersuchung, Blutuntersuchungen sowie eines Fragebogens (siehe Anhang) und einer Abdomensonografie in den Räumlichkeiten der Klinik für Gastroenterologie und gastrointestinale Onkologie der UMG (siehe Abbildung 3).

Im Anschluss an die Rekrutierungsphase vom 05.01.2016 - 31.12.2016 folgte eine Nachbeobachtungsphase von bis zu 12 Monaten, in der endgültige Diagnosen gestellt, bzw. weiterführende Untersuchungsergebnisse gewonnen werden konnten. Erfolgte im Rahmen der Überwachung/Behandlung des Patienten bis spätestens 12 Monate nach Erstvorstellung eine Wiedervorstellung im LZG, wurden anthropometrische, laborchemische und apparative Parameter sowie eine erneute Lifestyleanamnese als Verlaufsparameter erneut erhoben. 


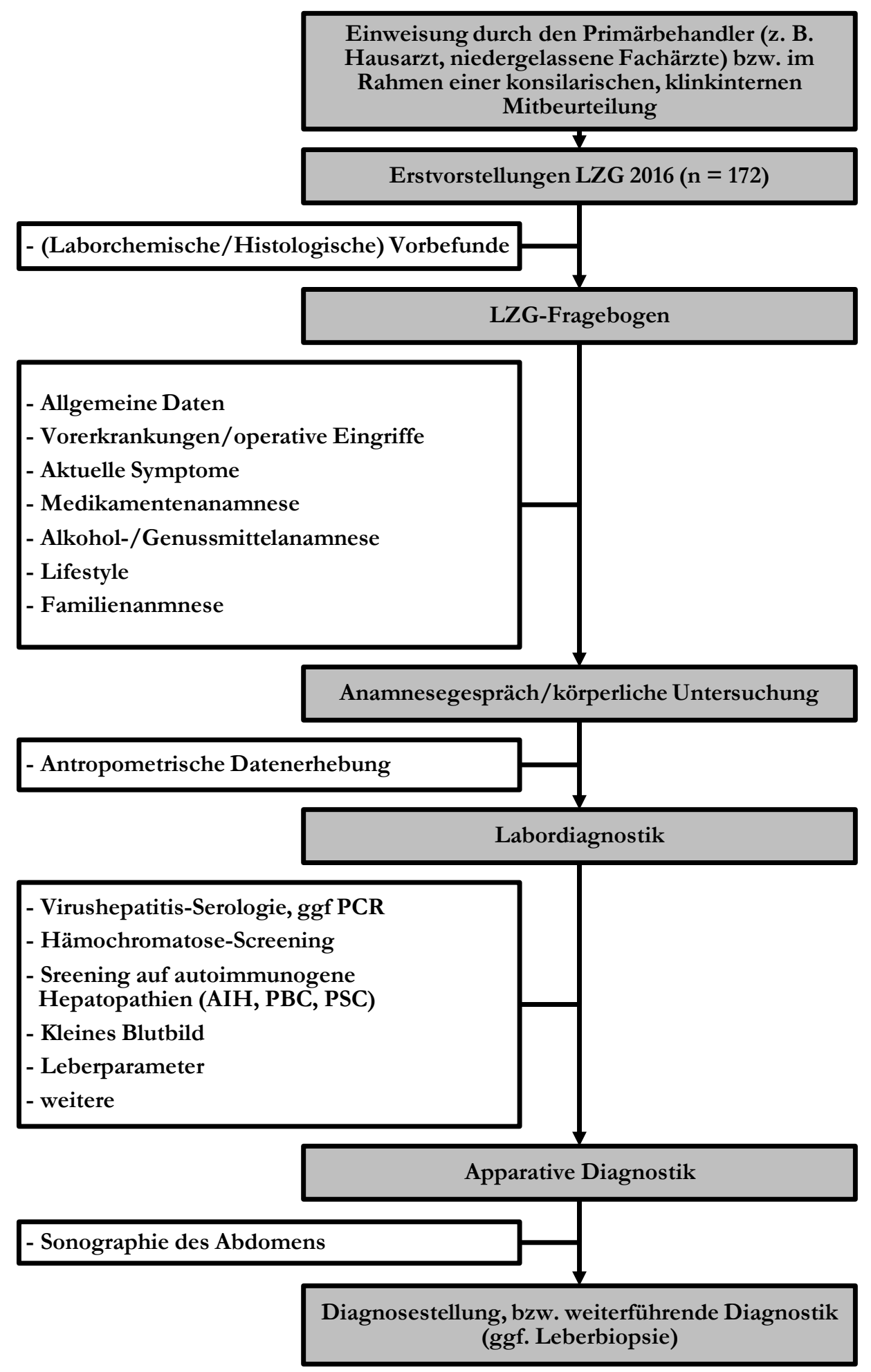

Abbildung 3: Diagnostischer Pfad im LZG Dargestellt ist der übliche Diagnosepfad für Neuvorstellungen in der Leberambulanz des Leberzentrum Göttingens.

AIH: Autoimmunhepatitis, LZG: Leberzentrum Göttingen, NAFLD: non-alcoholic fatty liver disease, PBC: Primär billäre Cholangitis, PCR: polymerase chain reaction; PSC: Primär sklerosierende Cholangitis 


\subsubsection{Anamnesegespräch/Patientenfragebogen}

Im Rahmen des Anamnesegespräches bei Erstkontakt erfolgte im Rahmen dieser Studie zusätzlich mit einem erfahrenen Hepatologen eine erweitere Lifestyle-Anamnese (s. u.). Das Anamnesegespräch mit dem ärztlichen Mitarbeiter diente in erster Linie dazu, die mit dem Patientenfragebogen des LZG (s. Anhang) erhobenen Daten zu verifizieren bzw. präzisieren sowie eine strukturierte Vorgeschichte des Patienten zu erhalten.

Der Patientenfragebogen wurde von den Patienten bereits vor Vorstellung in der UMG, spätestens jedoch vor der ersten Kontaktaufnahme ausgefüllt und während der Konsultation mit einem ärztlichen Mitarbeiter besprochen und ergänzt. Bestanden seitens des Patienten bzw. des Untersuchers Unklarheiten, so konnten diese im Arztgespräch geklärt werden.

\subsubsection{Suchtmittelanamnese}

Mithilfe des verwendeten Fragebogens und des Anamnesegespräches wurde das Suchtmittelverhalten der Patienten ermittelt. Auf Basis der Patientenangaben wurde so der durchschnittliche Tageskonsum reinen Ethanols (g/d) berechnet. Ein Wert von mehr als $20 \mathrm{~g} / \mathrm{d}$ (Männer) bzw. $10 \mathrm{~g} / \mathrm{d}$ (Frauen) stellte, gemäß der aktuellen Leitlinie, differentialdiagnostisch ein Ausschlusskriterium für die Diagnose einer NAFLD dar (Roeb 2015). Entsprechende Patienten wurden u. a. der Gruppe der AFLD zugeordnet (s. u.).

Die Evaluierung des aktuellen bzw. früheren Rauchverhaltens des Patienten erfolgte über die Bestimmung der Dauer des Konsums (Jahre) bzw. der täglichen Menge (Päckchen/d). Aus diesen erfolgte die Bestimmung der Rauchbelastung in pack years (py) (Konsumjahre x durchschnittliche Anzahl an konsumierten Zigarettenschachteln pro Tag).

Die Patienten wurden zusätzlich über den Konsum anderer Suchtmittel befragt.

\subsubsection{Lifestyle}

Mithilfe eines strukturierten Fragebogens (s. Anhang) und im Rahmen des Anamnesegespräches wurden dezidierte Lebensstilfaktoren ermittelt. So wurde der durchschnittliche tägliche Obstkonsum in Portionen pro Tag ermittelt, wobei eine Portion pragmatisch als Volumenäquivalent eines Apfels definiert wurde. Darüber hinaus wurde der durchschnittliche Softdrink- und Kaffeekonsum dokumentiert. Der Kaffeekonsum wurde in Portionen pro Tag ermittelt, wobei eine Portion als eine Menge von ca. $150 \mathrm{ml}$ definiert wurde. Zusätzlich wurden die durchschnittlichen Tage pro Woche mit sportlicher Betätigung, definiert als moderate oder schwere körperliche Aktivität von mindestens 30 Minuten, ermittelt. 


\subsubsection{Anthropometrische Datenerhebung}

Die anthropometrische Datenerhebung umfasste die standardisierte Bestimmung des Körpergewichts bzw. der Körpergröße. Dies erfolgte durch Messung durch das ärztliche Personal. Im Anschluss wurde der body mass index (BMI) mittels der Formel Körpermasse (kg) / (Körperlänge (m) x Körperlänge (m)) berechnet und als Wert in der Einheit $\mathrm{kg} / \mathrm{m}^{2}$ dokumentiert. Im Folgenden diente dieser zur Subgruppierung der NAFLDPatienten nach oben genannter Definition (siehe Abbildung 2).

\subsubsection{Körperliche Untersuchung}

Die körperliche Untersuchung wurde durch einen erfahrenen ärztlichen Mitarbeiter durchgeführt. Im Fokus stand insbesondere die Untersuchung auf klinische Anzeichen einer Hepatopathie. Zusätzlich erfolgte u. a. eine nichtinvasive Blutdruckmessung des Patienten durch Messung nach Riva-Rocci. Die Angabe der systolischen und diastolischen Blutdruckwerte erfolgte in Millimeter Quecksilbersäule ( $\mathrm{mmHg}$ ). Gemessen wurde i. d. R. einseitig.

\subsubsection{Labordiagnostik}

Für die erforderliche Diagnostik und Therapie(-einleitung) im Rahmen des Kontakts mit dem LZG wurden bereits im hausärztlichen Setting diverse Parameter erhoben, die während des EK ggf. ergänzt wurden (siehe Tabelle 3).

\subsubsection{Apparative Diagnostik}

Alle eingeschlossenen Patienten erhielten eine Sonographie des Abdomens. Diese wurde im Rahmen des Erstkontakts bzw. der Wiedervorstellungen der Patienten von einem erfahrenen Sonographeur unter Nutzung eines GE Logiq E9® in Kombination mit einem Konvexschallkopf durchgeführt. 
Tabelle 3: Laborchemische Referenzbereiche

\begin{tabular}{|c|c|c|c|}
\hline Laborparameter & Referenzbereich & Einheit & Material \\
\hline \multicolumn{4}{|l|}{ Kleines Blutbild } \\
\hline Hämoglobin & $11,5-17,5$ & $\mathrm{~g} / \mathrm{dl}$ & EDTA \\
\hline Hämatokrit & $35-51$ & $\%$ & EDTA \\
\hline Erythrozyten & $3,9-5,9$ & $10^{6} / \mu \mathrm{l}$ & EDTA \\
\hline $\mathrm{MCV}$ & $81-95$ & $\mathrm{fl}$ & EDTA \\
\hline Leukozyten & $4-11$ & $10^{3} / \mu \mathrm{l}$ & EDTA \\
\hline Thrombozyten & $150-350$ & $10^{3} / \mu \mathrm{l}$ & EDTA \\
\hline \multicolumn{4}{|l|}{ Leberparameter } \\
\hline Bilirubin (gesamt) & $\leq 1,2$ & $\mathrm{mg} / \mathrm{dl}$ & Li-Heparin \\
\hline AST & $\leq 35$ & $\mathrm{U} / 1$ & Li-Heparin \\
\hline ALT & $\leq 45$ & $\mathrm{U} / 1$ & Li-Heparin \\
\hline De-Ritis-Quotient & & & berechnet \\
\hline gGT & $9-64$ & $\mathrm{U} / 1$ & Li-Heparin \\
\hline $\mathrm{AP}$ & $40-150$ & $\mathrm{U} / 1$ & Li-Heparin \\
\hline Albumin & $3,4-5,0$ & $\mathrm{~g} / \mathrm{dl}$ & Li-Heparin \\
\hline Quick & $80-130$ & $\%$ & Citratblut \\
\hline INR & & & Citratblut \\
\hline Bilirubin (gesamt) & $\leq 1,2$ & $\mathrm{mg} / \mathrm{dl}$ & Li-Heparin \\
\hline AST & $\leq 35$ & $\mathrm{U} / 1$ & Li-Heparin \\
\hline ALT & $\leq 45$ & $\mathrm{U} / 1$ & Li-Heparin \\
\hline \multicolumn{4}{|c|}{ Weitere Laborparameter } \\
\hline Kreatinin & $0,5-1,2$ & $\mathrm{mg} / \mathrm{dl}$ & Li-Heparin \\
\hline HDL* & $>40$ & $\mathrm{mg} / \mathrm{dl}$ & Li-Heparin \\
\hline LDL* & $<160$ & $\mathrm{mg} / \mathrm{dl}$ & Li-Heparin \\
\hline Triglyceride* & $\leq 200$ & $\mathrm{mg} / \mathrm{dl}$ & Li-Heparin \\
\hline
\end{tabular}

Angegeben sind die erhobenen Laborparameter und deren Referenzbereiche sowie das verwendete Material.

* Nicht Bestandteil des ursprünglichen Studienplans. Es erfolgte die retrospektive Auswertung der ermittelten Parameter.

ALT: Alanin-Aminotransferase, AP: alkalische Phosphatase, AST: Aspartat-Aminotransferase, EDTA: Ethylendiamintetraessigsäure, gGT: Gamma-Glutamyltransferase, HDL: high density lipoprotein, INR: international normalized ratio, LDL: high density lipoprotein, Li-Heparin: Lithium-Heparin, MCV: mean corpuscular volume 


\subsubsection{Definition NAFLD}

Die Diagnose „NAFLD“ konnte sowohl invasiv, d.h. histologisch, als auch nichtinvasiv gestellt werden. Die Sonographie gilt aktuell als das bestgeeignetste, nichtinvasive Verfahren zur Detektion einer Steatosis (Weiss 2014). Bei der sonographischen Beurteilung wird die Echogenität der Leber mit der des Nierenparenchyms verglichen. Hierzu erfolgt die Einstellung einer Längsschnittebene, bei der Leber und Niere nebeneinander zur Darstellung kommen. Physiologisch stellen sich Leber- und Nierenparenchym isoechogen dar. Zeigt sich das Leberparenchym im Vergleich hyperechogen, d.h. heller, liegt eine sonographische Fettleber vor.

Zusätzlich musste eine sekundäre Fettleber weitestgehend ausgeschlossen werden. Hierbei wird nach Ursachen für sekundäre Fettlebern gefahndet (s. o.). Dies umfasst u. a. den Ausschluss von viralen Hepatitiden (Serologie und ggf. PCR), eines Morbus Wilson (Coeruloplasmin), einer Hämochromatose (Ferritin, Transferrinsättigung, ggf. HFEGenotypisierung), cholestatischer Lebererkrankungen (primär sklerosierende Cholangitis, primär biliäre Cholangitis) bzw. Autoimmunhepatitiden (IgG, IgM, Proteinelektrophorese, Autoantikörper-Panel (ANA, SMA, ANCA, AMA-M2, LKM, LC-1, SLA)), ggf. lysosomaler Speicherkrankheiten (z. B. LAL-Defizienz, M. Gaucher, M. Fabry) (siehe Tabelle 4) und der AFLD (Anamnese, ggf. Urin-EtG und EtS bzw. CDT). Die Bestimmung dieser Parameter erfolgte zum großen Teil durch den Primärbehandler/Zuweisenden und wurde bei Bedarf im LZG komplettiert werden.

\subsubsection{Follow-up}

Nach Erstvorstellung des Patienten erfolgte bei sämtlichen „NAFLD“-Patienten nach Erhebung der Lifestylefaktoren eine standardisierte Intervention im Sinne einer Empfehlung zur Lifestylemodifikation (s.u.).

Bei Wiedervorstellung sollte eine erneute Messung des Körpergewichts mit Berechnung des BMI sowie eine Ermittlung der Serum-ALT/-gGT durchgeführt werden. Aus dem Vergleich zwischen EK und WV konnten im Verlauf das $\Delta$ BMI, $\Delta$ ALT bzw. $\Delta$ gGT für die Gruppen „lean-NAFLD“ und „,non-lean-NAFLD“ gebildet werden. Diese beschreiben die absolute Wertdifferenz zwischen EK und WV.

Im Rahmen der Follow-up-Auswertung wurden zusätzlich anhand der messbaren und damit objektivierbaren Entwicklung des BMI die Vergleichsgruppen „Gewichtsverlust“ und „kein Gewichtsverlust" gebildet und ebenfalls die Veränderungen des BMI, der ALT und der gGT untersucht.

\subsubsection{Empfehlung zur moderaten Körpergewichtsreduktion}

Die Patienten wurden motiviert, eine moderate Körpergewichtsreduktion, von ca. ein bis zwei Kilogramm bis zur erneuten, geplanten Wiedervorstellung (in der Regel nach sechs Monaten), anzustreben. Es wurde auf einen starken, positiven Effekt bereits geringer 
Gewichtsreduktionen auf das Ausmaß der Fettleber und deren Entzündungsaktivität, bzw. das Entzündungsrisiko in aktuellen Studien hingewiesen.

\subsubsection{Empfehlung zur vermehrten körperlichen Betätigung}

Die Patienten wurden zu vermehrter sportlicher Betätigung motiviert. Es wurde auf einen nachweisbaren, positiven Effekt auf die Entzündungsaktivität und das Ausmaß der Leberverfettung, insbesondere im Rahmen von Fettlebererkrankungen, in aktuellen Studien hingewiesen.

\subsubsection{Empfehlung zur Diätumstellung}

Den Patienten wurde empfohlen sich bewusst zu ernähren und die Inhaltsstoffe ihrer Nahrungsmittel kritisch zu prüfen, um insbesondere Zuckerquellen zu identifizieren und den täglichen Zuckerkonsum zu reduzieren. Insbesondere wurde empfohlen, Süßigkeiten und Softdrinks gänzlich zu meiden. Sekundär wurde auch empfohlen, die Fettaufnahme zu reduzieren, bzw. insbesondere gesättigte Fettsäuren zu meiden.

\subsubsection{Empfehlung zur Alkoholreduktion bzw. -karenz}

Ergänzend wurde allen Patienten eine Reduktion eines bestehenden Alkoholkonsums bzw. eine möglichst vollständige Alkoholkarenz angeraten.

\subsection{Statistische Auswertung}

Der Aufbau der Patientendatenbank erfolgte mittels Microsoft Excel 2016® bzw. Microsoft Excel 2013®. Die statische Auswertung und Erstellung von Grafiken wurde mittels graphpad Prism 7® vorgenommen. Testanalytisch kam für Daten auf Rationalskalenniveau der Kruskal-Wallis-Test zum Vergleich von mehr als zwei Gruppen zur Anwendung. Wurden zwei Gruppen miteinander verglichen, wurde der Mann-Whitney-Test angewendet. In Fällen in denen Parameter einer Gruppe zwischen EK und WV verglichen werden sollten, fand der Wilcoxon matched pairs-Test Anwendung.

Parameter auf Ordinalskalenniveau wurden mithilfe des chi-square-Tests verglichen.

Das allgemeine Signifikanzniveau wurde für diese Studie mit $\mathrm{p}<0,05$ festgelegt. Relevante Signifikanzen werden in den Abbildungen nach folgendem Schema angegeben: „*“ für ein

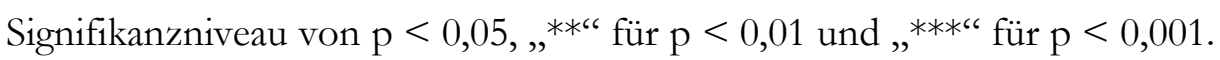




\section{$3 \quad$ Ergebnisteil}

\subsection{Kollektivübersicht}

Insgesamt stellten sich 213 Patienten im Jahr 2016 erstmalig im Leberzentrum Göttingen vor. Nach Ausschluss von 41 Patienten ohne Hinweis für eine aktive Hepatopathie zum Zeitpunkt des Einschlussendes, konnten 172 Patienten für diese Analyse berücksichtigt werden (siehe Abbildung 4). Vier Patienten willigten nicht in die Verwendung Ihrer Daten ein und wurden entsprechend ausgeschlossen.

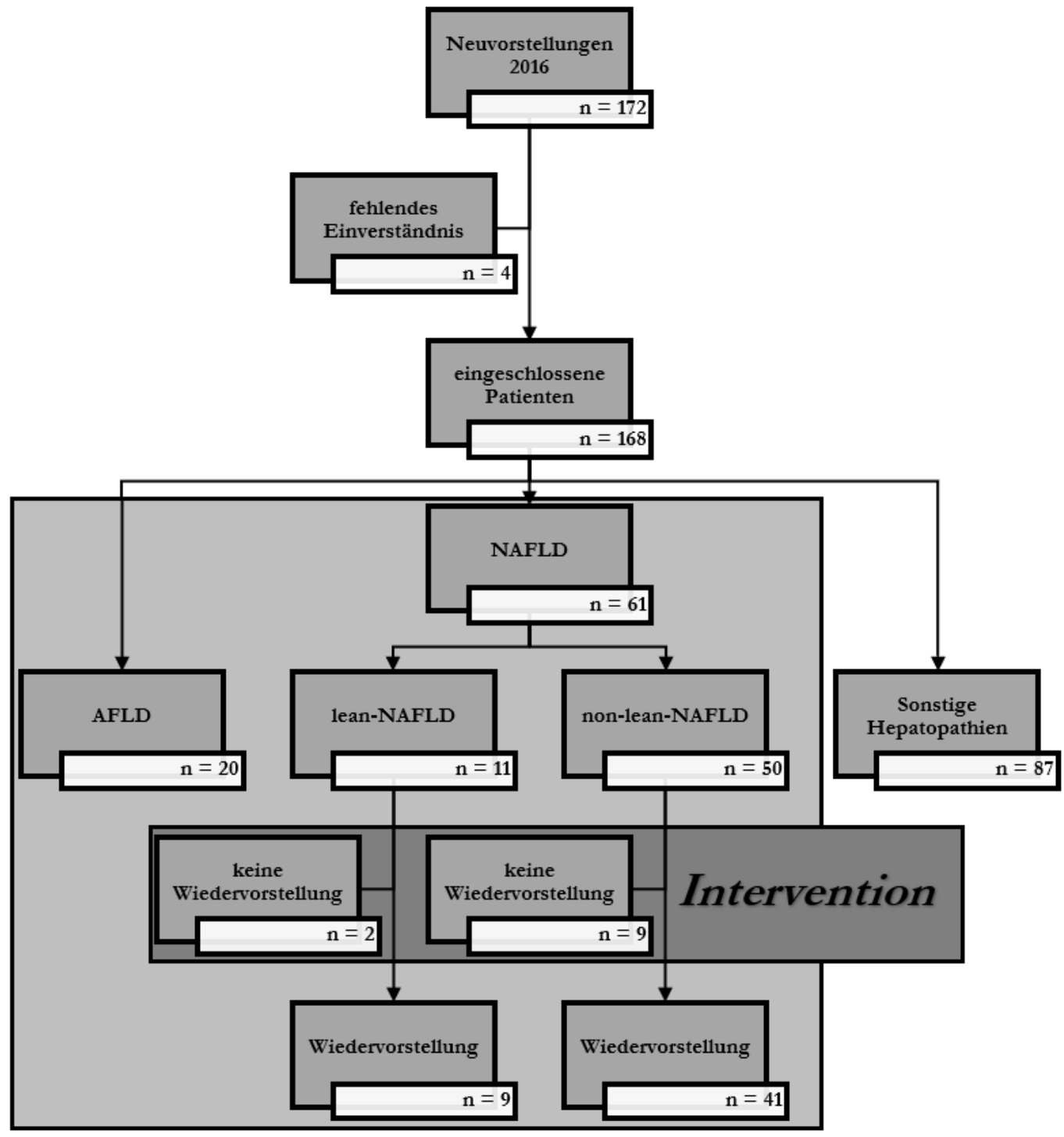

Abbildung 4: Studienkollektiv und Subgruppierungen Das Studienkollektiv umfasst primär 172 Patienten. Nach Ausschluss von vier Patienten (fehlende Einwilligung) konnten 168 Patienten in die Studie eingeschlossen werden. Davon wiesen 20 eine „AFLD“, 61 eine „NAFLD“ und 87 eine „sonstige Hepatopathie“ auf. Die „NAFLD“-Gruppe wurde anhand des BMI in die Gruppen ,lean-NAFLD“ ( $\mathrm{n}=11)$ und die ,non-leanNAFLD“ ( $n=50)$ eingeteilt, bei denen es nach Intervention zu neun bzw. 41 Wiedervorstellungen kam. 
Nach Diagnosestellung durch erfahrene Hepatologen des LZG wurden folgende Gruppen gebildet: 61 (36,31 \%) Patienten mit einer „NAFLD“, 20 mit einer „AFLD“ (11,90\%) sowie 89 „sonstige Hepatopathie“ (51,79\%). Die „sonstigen Hepatopathien“ wurden für die Analyse nicht weiter berücksichtigt (siehe Tabelle 4). Die Subgruppe „kombinierte Hepatopathien“ umfasst Patienten bei denen mehr als eine Lebererkrankung vorlag.

Tabelle 4: „Sonstige Hepatopathien“

\begin{tabular}{|c|c|}
\hline Gesamt & $\mathrm{n}=87$ \\
\hline Virale Hepatitiden & $\mathrm{n}=30$ \\
\hline $\mathrm{HBV}$ & $\mathrm{n}=21$ \\
\hline $\mathrm{HCV}$ & $\mathrm{n}=9$ \\
\hline Kombinierte Hepatopathien & $\mathrm{n}=17$ \\
\hline Autoimmune cholestatische Hepatopathien & $\mathrm{n}=15$ \\
\hline PBC & $\mathrm{n}=10$ \\
\hline PSC & $\mathrm{n}=4$ \\
\hline Sekundär sklerosierende Cholangitis & $\mathrm{n}=1$ \\
\hline Medikamentös-toxische Hepatopathie & $\mathrm{n}=5$ \\
\hline Autoimmunhepatitis & $\mathrm{n}=3$ \\
\hline Hämangiom & $\mathrm{n}=3$ \\
\hline Cirrhose cardiaque & $\mathrm{n}=2$ \\
\hline Leberzysten & $\mathrm{n}=2$ \\
\hline Alpha-1-Antitrypsin-Mangel & $\mathrm{n}=1$ \\
\hline Amöbenabszess & $\mathrm{n}=1$ \\
\hline Begleithepatitis & $\mathrm{n}=1$ \\
\hline Budd-Chiari-Syndrom & $\mathrm{n}=1$ \\
\hline DHC-Stenose & $\mathrm{n}=1$ \\
\hline Fokale noduläre Hyperplasie & $\mathrm{n}=1$ \\
\hline Glykogenose & $\mathrm{n}=1$ \\
\hline HFE-Compound-Heterozygotie & $\mathrm{n}=1$ \\
\hline Morbus Wilson & $\mathrm{n}=1$ \\
\hline Peliosis hepatis & $\mathrm{n}=1$ \\
\hline
\end{tabular}

Insgesamt 87 Patienten wurden der Gruppe „Sonstige Hepatopathie“ zugeordnet. Die Auflistung erfolgt in absteigender Reihenfolge nach ihrer absoluten Häufigkeit.

DHC: Ductus hepaticus communis, HBV: Hepatitis-B-Virus, HCV: Hepatitis-C-Virus, HFE: human hemochromatosis gene, PBC: primär billäre Cholangitis, PSC: primär sklerosierende Cholangitis 


\subsection{Differenzierbarkeit von AFLD und NAFLD anhand klinischer Routineparameter}

Eine klinische Herausforderung stellt die Differenzierung von NAFLD und AFLD dar. Dies gilt insbesondere für Fälle, in denen ein Patient nicht offen über seinen tatsächlichen Alkoholkonsum spricht. Liegt ein Alkoholkonsum nur kurz zurück, lässt sich dieser in der Regel durch (etablierte) Urin- oder Blutanalysen nachweisen (s. o.). Allerdings beweisen einzelne Nachweise keinen chronischen Alkoholkonsum. Fraglich bleibt, inwieweit eine Differenzierung dieser Hepatopathien anhand von Routineblutparametern möglich ist.

Bei Erstkontakt erfolgte aus diesem Grund die Einteilung in die Gruppen „AFLD“ ( $\mathrm{n}=20$, 24,69 \%) und „NAFLD“ ( $\mathrm{n}=61,75,31 \%)$. Bei Letzteren erfolgte vor der statistischen Auswertung die Subdifferenzierung in normalgewichtige, d.h. „lean-NAFLD“-Probanden $\left(\mathrm{BMI}<25 \mathrm{~kg} / \mathrm{m}^{2}\right)(\mathrm{n}=11,13,58 \%)$ und Patienten mit einem erhöhten BMI $\left(\geq 25 \mathrm{~kg} / \mathrm{m}^{2}\right)$ ( $\mathrm{n}=50,61,73 \%$ ). Diese werden gemäß o. g. Definition als „,non-lean-NAFLD“-Patienten bezeichnet.

\subsubsection{Epidemiologische und anthropometrische Charakteristika des Kollektivs}

Das durchschnittliche Lebensalter unterscheidet sich signifikant zwischen den untersuchten Gruppen ( $(\mathrm{p}<0,01) \quad$ (siehe Tabelle 5). „Lean-NAFLD“-Patienten $\quad(\mathrm{n}=11$, $39,55 \pm 14,41$ Jahre) sind jünger als Probanden sowohl der ,non-lean-NAFLD“- $(\mathrm{n}=50$, $50,92 \pm 15,07$ Jahre $)(p=0,03)$ als auch der „AFLD“-Gruppe $(\mathrm{n}=20,56,10 \pm$ 9,78 Jahre $)$ $(\mathrm{p}<0,01)$. Ein Unterschied zwischen „non-lean“- und „AFLD“-Patienten liegt hingegen nicht vor $(\mathrm{p}=0,16)$.

Bezüglich der Geschlechterverteilung sind die Gruppen nicht unterschiedlich zusammengesetzt ( $\mathrm{p}=0,17$ ) (siehe Tabelle 6). In der „lean-NAFLD“ und „,non-leanNAFLD“-Gruppe sind 45 \% bzw. 48 \% der Probanden männlich, während dies auf 70 \% der „AFLD“-Patienten zutrifft.

„Lean-NAFLD“-Patientin ( $\mathrm{n}=11,22,97 \pm 1,64 \mathrm{~kg} / \mathrm{m}^{2}$ ) weisen geringere BMI-Werte als „,non-lean-NAFLD“-Patienten $\left(\mathrm{n}=50,31,38 \pm 3,94 \mathrm{~kg} / \mathrm{m}^{2}\right)$ auf $(\mathrm{p}<0,001)$. Sie sind ebenfalls im Mittel leichter als die „AFLD“-Probanden ( $\mathrm{n}=20,28,82 \pm 5,97 \mathrm{~kg} / \mathrm{m}^{2}$ ) $(p<0,01)$, welche ihrerseits leichter als ,non-lean-NAFLD“-Patienten sind $(p=0,02)$. 
Tabelle 5: Epidemiologische Charakteristika des Kollektivs

\begin{tabular}{|c|c|c|c|c|}
\hline & lean-NAFLD & non-lean-NAFLD & AFLD & p-Wert \\
\hline (n) & 11 & 50 & 20 & \\
\hline \multicolumn{5}{|l|}{ Alter [Jahre] } \\
\hline Mean & 39,55 & 50,92 & 56,10 & \multirow{4}{*}{$<0,009$} \\
\hline Median & 40,00 & 51,00 & 56,00 & \\
\hline Range & $18,00-58,00$ & $18,00-85,00$ & $37,00-73,00$ & \\
\hline $\mathrm{SD}$ & $\pm 14,41$ & $\pm 15,07$ & $\pm 9,78$ & \\
\hline \multirow[t]{2}{*}{$95 \% \mathrm{CI}$} & $29,86-49,23$ & $46,64-55,20$ & $51,52-60,68$ & \\
\hline & & \multicolumn{2}{|c|}{ lean-NAFLD vs. non-lean-NAFLD } & 0,026 \\
\hline \multicolumn{2}{|c|}{ Subgruppenvergleich } & \multicolumn{2}{|c|}{ lean-NAFLD vs. AFLD } & $<0,001$ \\
\hline & & \multicolumn{2}{|c|}{ non-lean-NAFLD vs. AFLD } & 0,160 \\
\hline \multicolumn{5}{|l|}{ Geschlecht } \\
\hline männlich (n) & 5 & 24 & 14 & \multirow{3}{*}{0,175} \\
\hline$[\%]$ & 45 & 48 & 70 & \\
\hline weiblich (n) & 6 & 26 & 6 & \\
\hline$[\%]$ & 55 & 52 & 30 & \\
\hline
\end{tabular}

Aufgeführt sind die Parameter Alter und BMI in den untersuchten Gruppen.

AFLD: alcoholic fatty liver disease, BMI: body mass index, NAFLD: non-alcoholic fatty liver disease

Sowohl der systolische $(p=0,17)$ als auch der diastolische $(p=0,15)$ und der errechnete mittlere arterielle Blutdruck (MAD) ( $\mathrm{p}=0,14)$ waren in allen Gruppen gleich (siehe Tabelle 6). Die erhobenen MAD liegen zwischen 110,1 \pm 14,7 $\mathrm{mmHg}$ bei „lean-NAFLD“ $(\mathrm{n}=11)$ und 120,3 $\pm 15,1 \mathrm{mmHg}$ bei „AFLD“ $(\mathrm{n}=50)(\mathrm{p}=0,10)$. 
Tabelle 6: Anthropometrische Charakteristika des Kollektivs

\begin{tabular}{|c|c|c|c|c|}
\hline & lean-NAFLD & non-lean-NAFLD & AFLD & p-Wert \\
\hline (n) & 11 & 50 & 20 & \\
\hline \multicolumn{5}{|l|}{ BMI $\left[\mathrm{kg} / \mathrm{m}^{2}\right]$} \\
\hline Mean & 22,97 & 31,38 & 28,82 & \\
\hline Median & 23,50 & 30,52 & 27,55 & $<0,001$ \\
\hline Range & $20,40-24,80$ & $25,71-41,73$ & $20,76-44,96$ & \\
\hline $\mathrm{SD}$ & $\pm 1,644$ & $\pm 3,938$ & $\pm 5,968$ & \\
\hline \multirow[t]{2}{*}{$95 \% \mathrm{CI}$} & $21,87-24,08$ & $30,27-32,50$ & $26,03-31,61$ & \\
\hline & & \multicolumn{2}{|c|}{ lean-NAFLD vs. non-lean-NAFLD } & $<0,001$ \\
\hline \multirow{2}{*}{\multicolumn{2}{|c|}{ Subgruppenvergleich }} & \multicolumn{2}{|c|}{ lean-NAFLD vs. AFLD } & 0,001 \\
\hline & & \multicolumn{2}{|c|}{ non-lean-NAFLD vs. AFLD } & 0,020 \\
\hline \multicolumn{5}{|l|}{ MAD [mmHg] } \\
\hline Mean & 110,1 & 120,3 & 115,3 & \\
\hline Median & 106,0 & 117,0 & 107,5 & 0,141 \\
\hline Range & $93,00-130,0$ & $90,00-151,0$ & $93,00-147,0$ & \\
\hline SD & $\pm 14,74$ & $\pm 15,07$ & $\pm 18,71$ & \\
\hline $95 \% \mathrm{CI}$ & $96,51-123,8$ & $115,8-124,8$ & $106,0-124,6$ & \\
\hline \multicolumn{5}{|c|}{ RR syst. $[\mathrm{mmHg}]$} \\
\hline Mean & 126,7 & 138,9 & 134,3 & \\
\hline Median & 126,0 & 134,0 & 123,0 & 0,173 \\
\hline Range & $104,0-150,0$ & $100,0-172,0$ & $106,0-175,0$ & \\
\hline $\mathrm{SD}$ & $\pm 16,21$ & $\pm 18,31$ & $\pm 23,45$ & \\
\hline $95 \% \mathrm{CI}$ & $111,7-141,7$ & $133,4-144,4$ & $122,6-145,9$ & \\
\hline \multicolumn{5}{|c|}{ RR diast. [mmHg] } \\
\hline Mean & 78,29 & 84,13 & 78,39 & \\
\hline Median & 73,00 & 85,00 & 75,50 & 0,152 \\
\hline Range & $65,00-101,0$ & $61,00-117,0$ & $60,00-96,00$ & \\
\hline $\mathrm{SD}$ & $\pm 5,17$ & $\pm 1,63$ & $\pm 2,59$ & \\
\hline $95 \% \mathrm{CI}$ & $65,63-90,94$ & $80,85-87,42$ & $72,93-83,84$ & \\
\hline
\end{tabular}

Aufgeführt sind die Parameter RR syst. bzw. diast. und der MAD in den untersuchten Gruppen.

AFLD: alcoholic fatty liver disease, MAD: mittlerer arterieller Druck, NAFLD: non-alcoholic fatty liver disease, RR syst./diast.: nichtinvasive Blutdruckmessung nach Riva-Rocci systolisch/diastolisch 


\subsubsection{Blutbildparameter}

Bezüglich der Blutbildparameter zeigen sich durchschnittlich normwertige Befunde in allen untersuchten (Sub-) Gruppen (siehe Tabelle 7).

Der mittlere Hämoglobinwert variiert zwischen 13,53 \pm 2,05 g/dl bei „AFLD“ ( $\mathrm{n}=20)$ und $14,35 \pm 1,44 \mathrm{~g} / \mathrm{dl}$ bei „,non-lean-NAFLD“ ( $\mathrm{n}=50)$. Die „lean-NAFLD“-Patienten ( $\mathrm{n}=11$, $13,72 \pm 1,63 \mathrm{~g} / \mathrm{dl})$ gruppieren sich zwischen diesen ein. Signifikante Unterschiede bestehen zwischen den Gruppen nicht $(\mathrm{p}=0,29)$.

Tabelle 7: Kleines Blutbild

\begin{tabular}{ccccc}
\hline \hline & lean-NAFLD & non-lean-NAFLD & AFLD & p-Wert \\
\hline (n) & $\mathbf{1 1}$ & $\mathbf{5 0}$ & $\mathbf{2 0}$ & \\
\hline Hämoglobin $[\mathbf{g} / \mathbf{d l}]$ & & & \\
Mean & 13,72 & 14,35 & 13,53 & 0,286 \\
Median & 13,50 & 14,65 & 14,10 & \\
Range & $11,5-16,50$ & $10,20-17,40$ & $8,800-15,80$ & \\
SD & $\pm 1,634$ & $\pm 1,440$ & $\pm 2,048$ & \\
$95 \%$ CI & $12,62-14,82$ & $13,94-14,76$ & $12,57-14,49$ & \\
\hline
\end{tabular}

Hämatokrit [\%]

$\begin{array}{ccccc}\text { Mean } & 40,80 & 42,70 & 39,98 & \\ \text { Median } & 41,40 & 42,95 & 41,25 & 0,059 \\ \text { Range } & 34,10-47,90 & 32,20-50,60 & 27,00-46,70 \\ \text { SD } & \pm 4,606 & \pm 3,981 & \pm 5,543 \\ 95 \% \text { CI } & 37,71-43,89 & 41,57-43,83 & 37,38-42,57\end{array}$

Erythrozyten $\left[10^{6} / \mu 1\right]$

\begin{tabular}{|c|c|c|c|c|}
\hline Mean & 4,628 & 4,811 & 4,294 & \\
\hline Median & 4,660 & 4,780 & 4,370 & 0,001 \\
\hline Range & $3,540-5,620$ & $3,500-5,870$ & $3,290-4,800$ & \\
\hline SD & $\pm 0,7174$ & $\pm 0,4640$ & $\pm 0,4534$ & \\
\hline $95 \% \mathrm{CI}$ & $4,146-5,110$ & $4,679-4,943$ & $4,082-4,506$ & \\
\hline \multirow{3}{*}{\multicolumn{2}{|c|}{ Subgruppenvergleich }} & \multicolumn{2}{|c|}{ lean-NAFLD vs. non-lean-NAFLD } & 0,292 \\
\hline & & \multicolumn{2}{|c|}{ lean-NAFLD vs. AFLD } & 0,122 \\
\hline & & \multicolumn{2}{|c|}{ non-lean-NAFLD vs. AFLD } & $<0,001$ \\
\hline
\end{tabular}




\begin{tabular}{|c|c|c|c|c|}
\hline & lean-NAFLD & non-lean-NAFLD & AFLD & p-Wert \\
\hline (n) & 11 & 50 & 20 & \\
\hline \multicolumn{5}{|l|}{$\operatorname{MCV}[\mathbf{f l}]$} \\
\hline Mean & 88,73 & 88,72 & 93,00 & \\
\hline Median & 89,00 & 89,00 & 94,50 & 0,020 \\
\hline Range & $83,00-96,00$ & $72,00-98,00$ & $77,00-108,0$ & \\
\hline SD & $\pm 4,496$ & $\pm 4,974$ & $\pm 7,921$ & \\
\hline \multirow[t]{2}{*}{$95 \% \mathrm{CI}$} & $85,71-91,75$ & $87,31-90,13$ & $89,29-96,71$ & \\
\hline & & \multicolumn{2}{|c|}{ lean-NAFLD vs. non-lean-NAFLD } & $<0,999$ \\
\hline \multirow{2}{*}{\multicolumn{2}{|c|}{ Subgruppenvergleich }} & \multicolumn{2}{|c|}{ lean-NAFLD vs. AFLD } & 0,112 \\
\hline & & \multicolumn{2}{|c|}{ non-lean-NAFLD vs. AFLD } & 0,008 \\
\hline
\end{tabular}

Leukozyten $\left[10^{3} / \mu 1\right]$

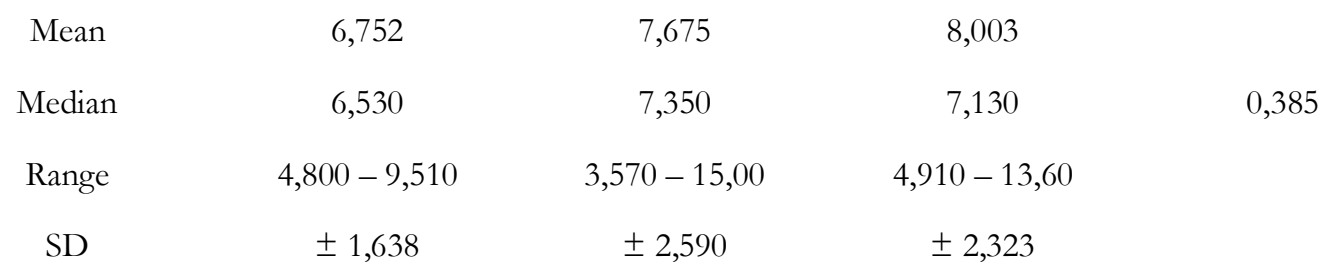

$95 \%$ CI $\quad 5,651-7,853 \quad 6,939-8,411 \quad 6,915-9,090$

Thrombozyten $\left[10^{3} / \mu 1\right]$

$\begin{array}{ccccc}\text { Mean } & 228,7 & 238,2 & 150,8 \\ \text { Median } & 239,0 & 226,5 & 140,0 & \\ \text { Range } & 168,0-301,0 & 48,00-433,0 & 69,00-353,0 & <0,001 \\ \text { SD } & \pm 45,81 & \pm 82,11 & \pm 63,88\end{array}$

$95 \%$ CI $\quad 198,0-259,5 \quad 214,9-261,5 \quad 120,9-180,6$

Subgruppenvergleich

lean-NAFLD vs. non-lean-NAFLD $\quad 0,714$

$\begin{array}{rr}\text { lean-NAFLD vs. AFLD } & <0,001 \\ \text { non-lean-NAFLD vs. AFLD } & <0,001\end{array}$

Aufgeführt sind die Parameter Hämoglobin, Hämatokrit, Erythrozyten und MCV

AFLD: alcoholic fatty liver disease, MCV: mean corpuscular volume, NAFLD: non-alcoholic fatty liver disease

Der mittlere Hämatokrit differiert zwischen den Patientengruppen ebenfalls nicht $(p=0,06)$. Er schwankt zwischen 39,98 \pm 5,54 \% bei „AFLD“ $(\mathrm{n}=20)$ und $42,70 \pm 3,98 \%$ bei ,nonlean-NAFLD“ ( $\mathrm{n}=50)$. Die „lean-NAFLD“-Probanden $(\mathrm{n}=11,40,80 \pm 4,61)$ gruppieren sich zwischen diesen ein. 
Die durchschnittliche Erythrozytenzahl unterscheidet sich im Subgruppenvergleich $(\mathrm{p}<0,01)$. Dies trifft im Detail für die ,non-lean-NAFLD“ ( $\left.\mathrm{n}=50,4,81 \pm 0,4610^{6} / \mu \mathrm{l}\right)$ und „AFLD“ ( $\left.\mathrm{n}=20,4,29 \pm 0,4510^{6} / \mu \mathrm{l}\right) \mathrm{zu}(\mathrm{p}<0,01)$. Die ,lean-NAFLD“ ( $\mathrm{n}=11$, $\left.4,63 \pm 0,7210^{6} / \mu \mathrm{l}\right)$ unterscheidet sich weder von der „AFLD“ $(\mathrm{p}=0,12)$ noch von der „,nonlean-NAFLD“ ( $p=0,29)$.

Das MCV variiert in den unterschiedlichen Gruppen $(\mathrm{p}=0,02)$. Ein signifikanter Unterschied liegt zwischen „non-lean-NAFLD“ ( $\mathrm{n}=50,88,72 \pm$ 4,97 fl) und „AFLD“ ( $\mathrm{n}=20,93,00 \pm 7,92 \mathrm{fl})$ vor $(\mathrm{p}=0,01)$. Die „lean-NAFLD“ $(\mathrm{n}=11,88,73 \pm 4,50 \mathrm{fl})$ unterscheidet sich weder von der ,non-lean-NAFLD“ ( $p>0,99)$ noch der „AFLD“ ( $p=$ $0,11)$.

Die Leukozytenzahl in den Gruppen weist weder eine Abweichung vom Normbereich noch signifikante Unterschiede untereinander auf $(\mathrm{p}=0,38)$. Der Mittelwert der „lean-NAFLD“Gruppe ( $\mathrm{n}=11,6,75 \pm 1,64 / \mu \mathrm{l})$ ist insgesamt am niedrigsten, während dieser bei „AFLD“Patienten $(n=20)$ bei $8,00 \pm 2,32103 / \mu$ l liegt.

Die durchschnittlichen Thrombozytenzahlen lagen in allen Gruppen innerhalb des laborseitig angegebenen Referenzbereichs. Die gemessenen Werte variieren allerdings in hochsignifikantem Ausmaß ( $p<0,001)$. Die „AFLD“-Gruppe $(n=20)$ ist mit einem Mittelwert von 150,8 \pm 63,88 103\% $\mu$ l noch grenzwertig normal. Die Gruppen „lean-NAFLD“ ( $\left.\mathrm{n}=11,228,7 \pm 45,8110^{3} / \mu \mathrm{l}\right)$ und ,non-lean-NAFLD“ ( $\mathrm{n}=50,238,2 \pm 82,1110^{3} / \mu \mathrm{l}$ ) weisen im direkten Vergleich deutlich höhere Werte auf (jeweils $\mathrm{p}<0,01$ ).

\subsubsection{Transaminasen}

Die Aspartat-Aminotransferase (AST) ist lediglich in der NAFLD-Subgruppe ,leanNAFLD“ ( $\mathrm{n}=11,42,45 \pm 12,64 \mathrm{U} / \mathrm{l}$ ) normwertig (siehe Abbildung 5). Die mittlere AST sowohl der ,non-lean-NAFLD“- (n = 50, 55,72 \pm 38,71 U/l) als auch der „AFLD“-Gruppe ( $\mathrm{n}=20,66,40 \pm 47,85 \mathrm{U} / \mathrm{l}$ ) überschreitet den laborseitig angegebenen Referenzbereich. Zwischen den Gruppen bestehen jedoch keine signifikanten Unterschiede ( $p=0,32)$.

Sämtliche Gruppen überschreiten den laborseitig angegebenen Referenzbereichs für die ALT. Bei „AFLD“- ( $\mathrm{n}=20,56,70 \pm 72,81 \mathrm{U} / \mathrm{l})$ und „lean-NAFLD“-Patienten ( $\mathrm{n}=11,52,82 \pm 19,03 \mathrm{U} / \mathrm{l})$ ist eine grenzwertige ALT-Erhöhung feststellbar, während diese bei „non-lean-NAFLD“ ( $\mathrm{n}=50,94,32 \pm 109,9$ U/l) höher ausfällt. Ein signifikanter Unterschied zwischen den untersuchten Gruppen liegt lediglich zwischen „,non-leanNAFLD“ und „AFLD“ vor $(\mathrm{p}<0,01)$.

Der de-Ritis-Quotient ist bei „lean-NAFLD“ ( $\mathrm{n}=11,0,93 \pm 0,58)$ und „non-lean-NAFLD“ ( $\mathrm{n}=50,0,75 \pm 0,32)$ normwertig. Im Gegensatz dazu ist bei „AFLD“ ( $\mathrm{n}=20,1,74 \pm 0,75)$ ein signifikant höherer Wert feststellbar ( $p=0,02$ bzw. $p<0,001)$. 
Aspartat-Aminotransferase (AST)
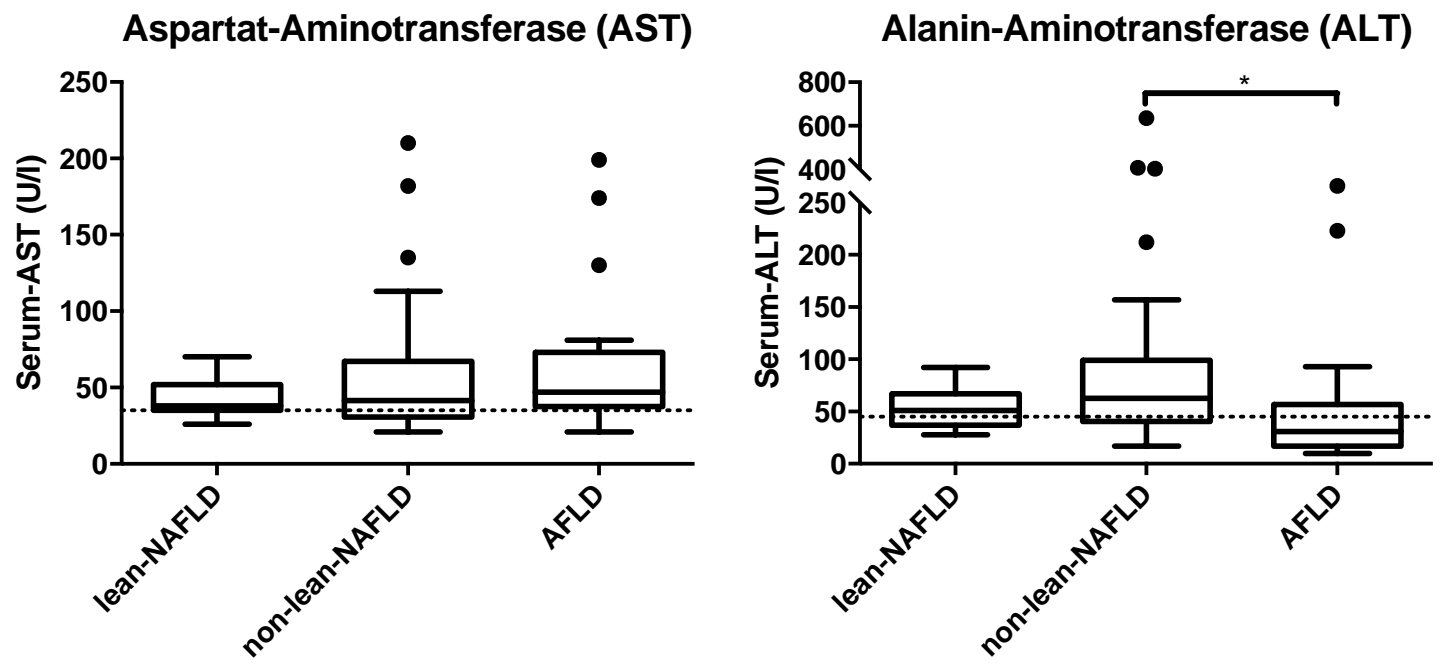

(a)

(b)

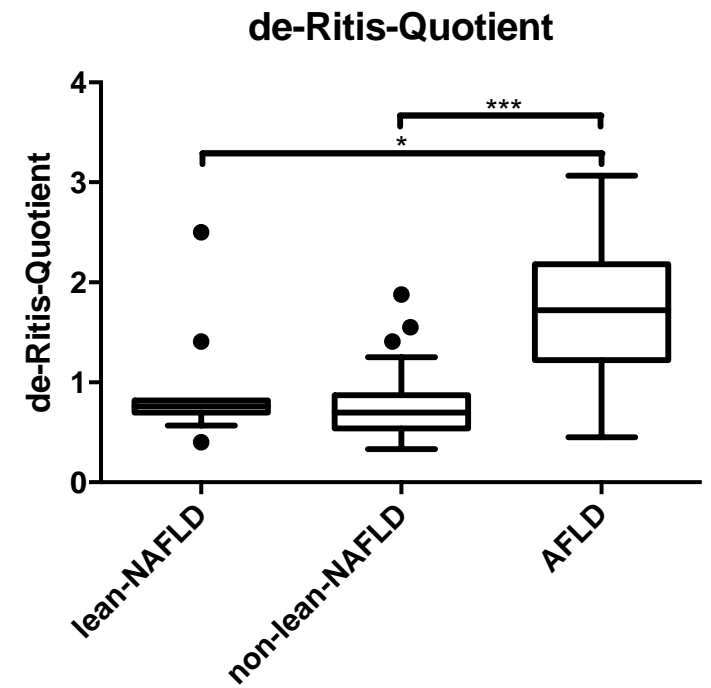

(c)

Abbildung 5: Transaminasen Die Graphen (a) - (c) zeigen die Parameter AST, ALT und den de-RitisQuotienten für die angegebenen Gruppen. Die jeweiligen gültigen Referenzbereiche sind durch die gestrichelten Linien hervorgehoben.

Die AST unterscheidet sich nicht zwischen den untersuchten Gruppen ( $p=0,32)$. Eine Unterscheidung der Gruppen bezüglich der ALT ist lediglich für den Vergleich zwischen „AFLD“ und „non-lean-NAFLD“ möglich ( $\mathrm{p}<0,01)$. Alle Gruppen überschreiten den laborseitig angegebenen ALT-Referenzwert. Der de-RitisQuotient ist bei „AFLD“ signifikant höher als in den Vergleichsgruppen $(\mathrm{p}=0,02$ bzw. $\mathrm{p}<0,001)$.

AFLD: alcoholic fatty liver disease, ALT: Alanin-Aminotransferase, AST: Aspartat-Aminotransferase, NAFLD: nonalcoholic fatty liver disease 


\subsubsection{Cholestaseparameter}

Die „AFLD“ ( $\mathrm{n}=20,1,45 \pm 0,95 \mathrm{mg} / \mathrm{dl}$ ) überschreitet im Gegensatz zu den ,non-leanNAFLD“-Patienten ( $\mathrm{n}=50,0,64 \pm 0,29 \mathrm{mg} / \mathrm{dl})(\mathrm{p}<0,001)$ und „lean-NAFLD“-Patienten $(\mathrm{n}=11,0,89 \pm 0,42 \mathrm{mg} / \mathrm{dl})(\mathrm{p}=0,93)$ den laborseitig angegebenen Referenzbereichs bezüglich des Bilirubins (siehe Abbildung 6).

Im Rahmen einer „AFLD“ ( $\mathrm{n}=20,279,5 \pm 207,1 \mathrm{U} / \mathrm{l})$ ist die gGT erhöht. Sie liegt signifikant über den Werten der ,lean-NAFLD“ $(n=11,123,6 \pm 134,6 \mathrm{U} / \mathrm{l})(\mathrm{p}=0,03)$ und „non-lean-NAFLD“ ( $\mathrm{n}=50,279,5 \pm 207,1 \mathrm{U} / \mathrm{l})(\mathrm{p}=0,01)$. Einen Unterschied innerhalb der „NAFLD“-Subgruppen ist hingegen nicht feststellbar $(\mathrm{p}>0,99)$.

Die durchschnittlichen AP-Spiegel lagen in allen Gruppen innerhalb des laborseitig angegebenen Referenzbereichs. „AFLD“-Patienten ( $\mathrm{n}=20,177,3 \pm$ 147,5 U/1) weisen signifikant höhere Werte als die „lean“- $(\mathrm{n}=11,87,82 \pm 28,39 \mathrm{U} / \mathrm{l})(\mathrm{p}=0,01)$ und „nonlean-NAFLD“-Patienten ( $\mathrm{n}=49,102,9 \pm 48,40 \mathrm{U} / \mathrm{l})(\mathrm{p}=0,01)$ auf. 


\section{Gesamt-Bilirubin}

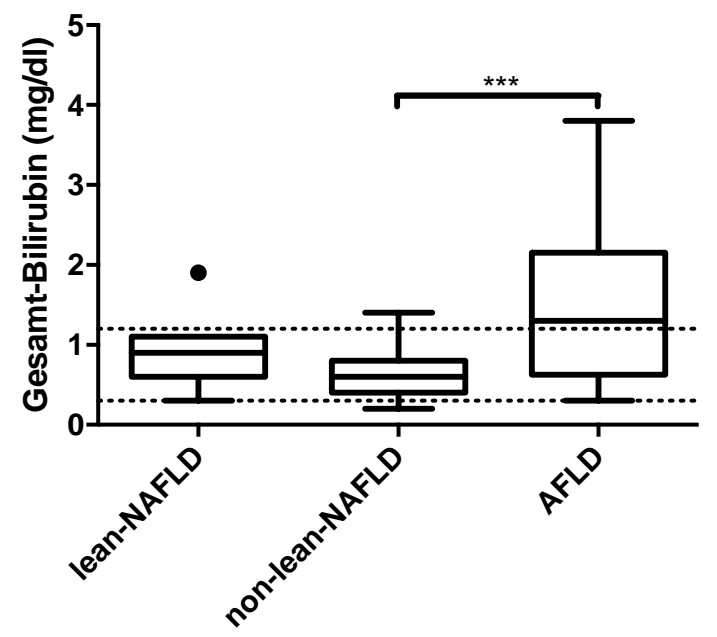

(a)

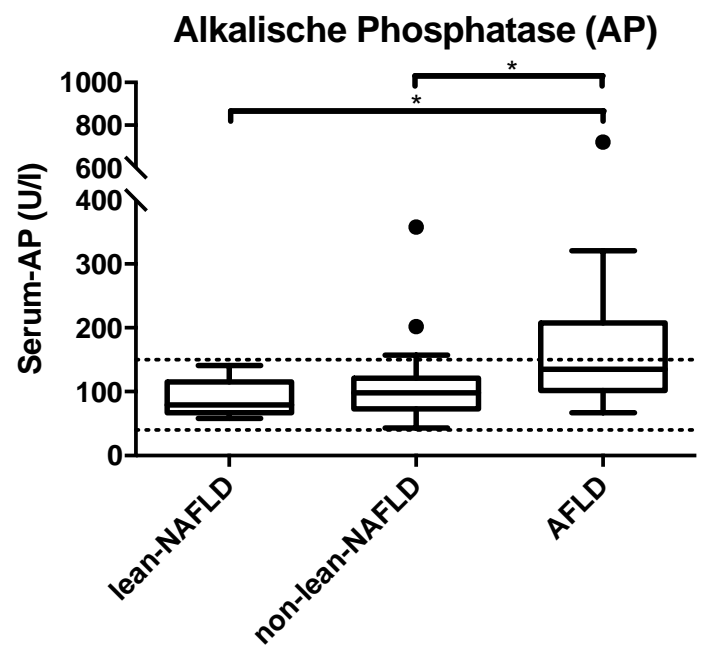

gamma-Glutamyltransferase (gGT)

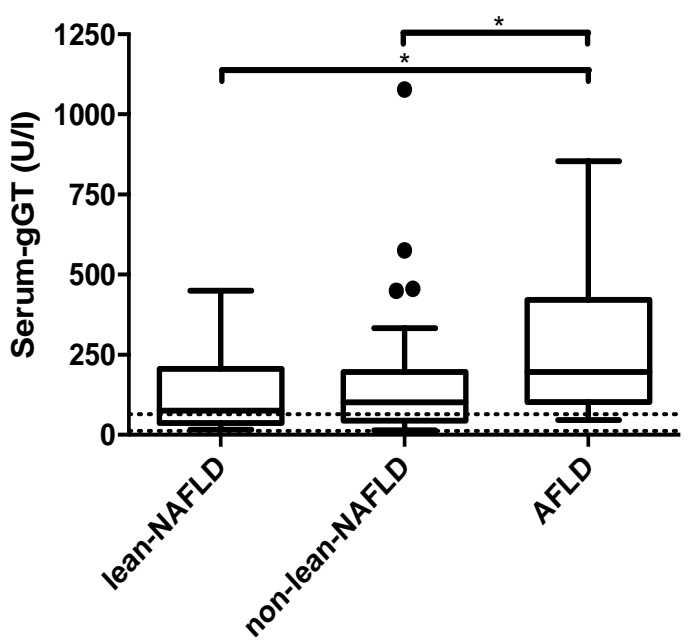

(b)

(c)

Abbildung 6: Cholestaseparameter Die Graphen (a) - (c) zeigen die Parameter Bilirubin, gGT und AP für die angegebenen Gruppen. Die jeweiligen gültigen Referenzbereiche sind durch die gestrichelten Linien hervorgehoben.

Der Bilirubinspiegel der „AFLD“-Gruppe überschreitet den Referenzbereich und liegt deutlich über denen der „non-lean-NAFLD“-Gruppe ( $\mathrm{p}<0,001)$. Ein Unterschied zur „lean-NAFLD“ besteht nicht $(\mathrm{p}=0,93)$. Die gGT-Spiegel sind bei „AFLD“-Patienten höher als bei „lean-NAFLD“ $(p=0,03)$ und ,non-lean-NAFLD“ $(\mathrm{p}=0,01)$. Diese Beobachtung trifft ebenso für die AP zu (jeweils $\mathrm{p}=0,01)$.

AFLD: alcoholic fatty liver disease, AP: alkalische Phosphatase, gGT: Gamma-Glutamyltransferase, NAFLD: nonalcoholic fatty liver disease 


\subsubsection{Lebersyntheseparameter}

Das Serumalbumin bei „AFLD“ ( $\mathrm{n}=20,3,36 \pm 0,63 \mathrm{~g} / \mathrm{dl})$ ist grenzwertig, $\mathrm{d}$. h. niedrig normal (siehe Abbildung 7). Im Vergleich zur „AFLD“ ist dieser Parameter sowohl bei „leanNAFLD“ ( $\mathrm{n}=11,4,18 \pm$ 0,30 g/dl) ( $\mathrm{p}<$ 0,001), als auch ,non-lean-NAFLD“ ( $\mathrm{n}=50,4,00$ $\pm 0,34 \mathrm{~g} / \mathrm{dl})(\mathrm{p}<0,001)$ signifikant höher. Die NAFLD-Subgruppenanalyse ergibt keine Unterschiede $(p=0,48)$.
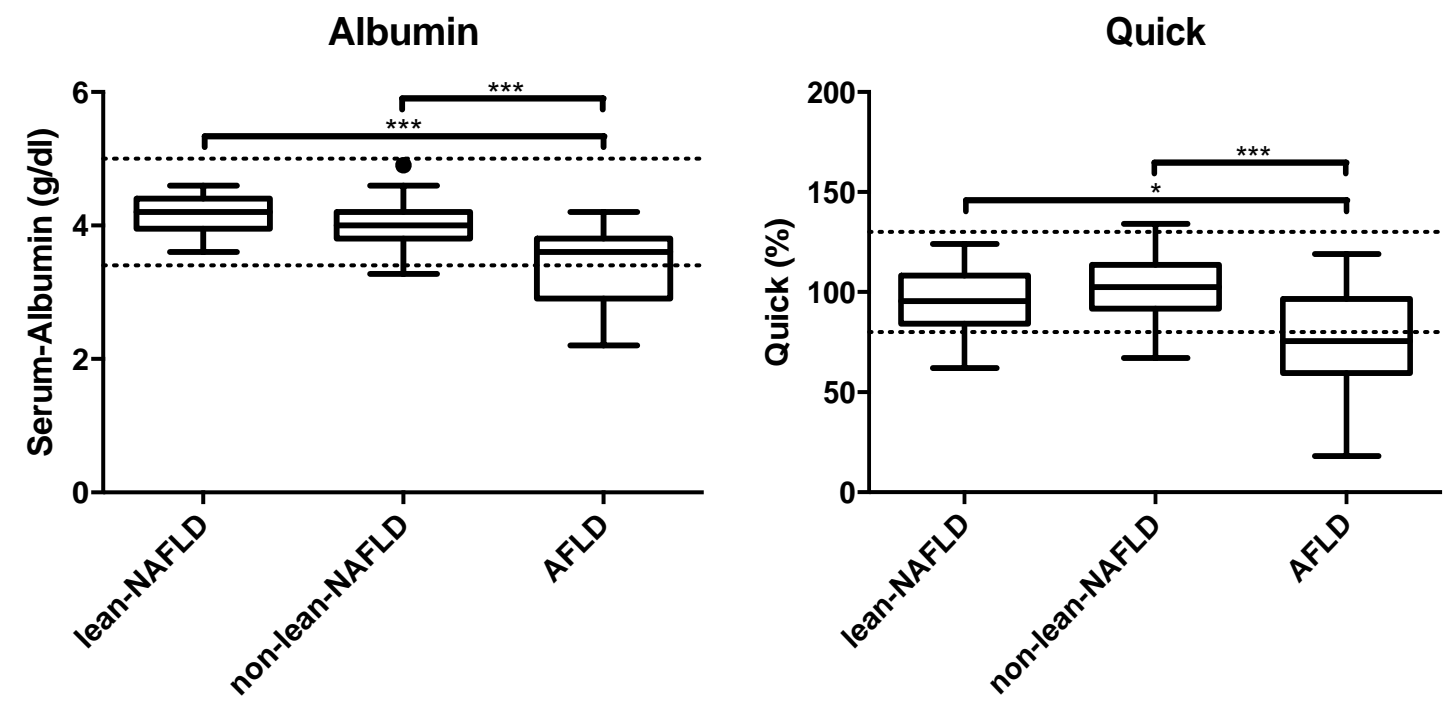

(a)

(b)

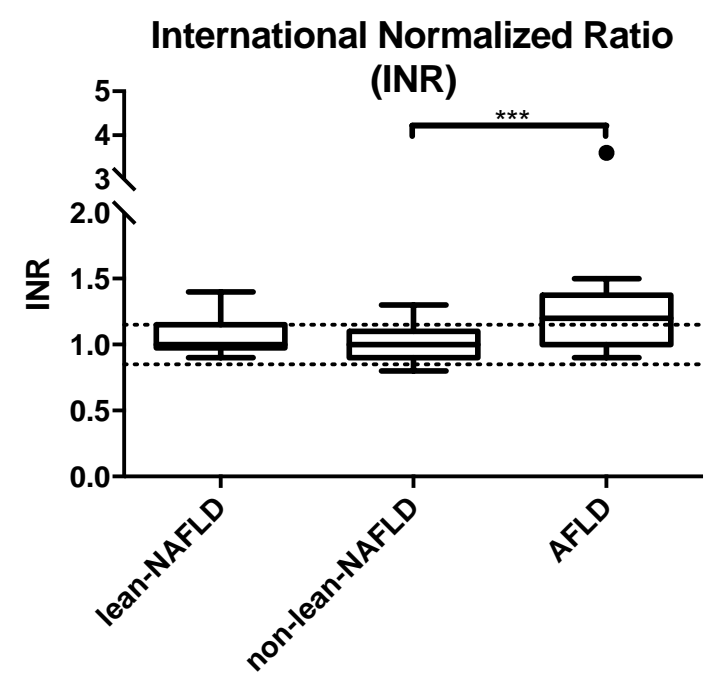

(c)

Abbildung 7: Lebersyntheseparameter Die Graphen (a) - (c) zeigen die Parameter Albumin, Quick und INR für die angegebenen Gruppen. Die jeweiligen gültigen Referenzbereiche sind durch die gestrichelten Linien hervorgehoben.

Der Albuminspiegel bei „AFLD“ liegt grenzwertig innerhalb des Normbereichs, jedoch signifikant niedriger als in den Vergleichsgruppen (jeweils p < 0,001). Der Quick- und INR-Wert sind bei „AFLD“ außerhalb des Normbereiches. 
Keiner der untersuchten Patienten erhielt Antikoagulantien, die den Quick- bzw. INR-Wert beeinflussen können (z. B. Phenprocoumon, Warfarin oder direkte orale Antikoagulantien (DOAKs)). Der mittlere Quick-Wert ist in den Gruppen mit Ausnahme der „AFLD“Kohorte ( $\mathrm{n}=20,77,5 \pm 23,44 \%$ ) normwertig. Sowohl bei Vorliegen einer „lean-NAFLD“ $(\mathrm{n}=11,94,60 \pm 19,68 \%)$, als auch ,non-lean-NAFLD“ $(\mathrm{n}=50,103,3 \pm 14,83 \%)$ sind diese höher. Die „NAFLD“-Subgruppen sind hinsichtlich ihres Quick-Wertes identisch $(\mathrm{p}=0,35)$.

Die INR bei „AFLD“-Patienten ( $\mathrm{n}=$ 20, 1,29 \pm 0,57) überschreitet den Referenzbereich knapp. Dieser wird weder von der „lean-NAFLD“- $(\mathrm{n}=11,1,06 \pm 0,16)$, noch „,non-leanNAFLD“-Gruppe ( $\mathrm{n}=50,0,99 \pm 0,10)$ überschritten. Diese Beobachtung erweist sich lediglich für den Vergleich zwischen „non-lean-NAFLD“ und „AFLD“ als signifikant $(\mathrm{p}<0,001)$.

\subsubsection{Nierenretentionsparameter}

Das Serum-Kreatinin ist in sämtlichen Gruppen normwertig und nicht signifikant unterschiedlich $(\mathrm{p}=0,22)$. Die durchschnittlich niedrigsten Werte werden im Rahmen einer „lean-NAFLD“ ( $\mathrm{n}=7,0,64 \pm 0,16 \mathrm{mg} / \mathrm{dl}$ ) beobachtet. Bei „non-lean-NAFLD“ ( $\mathrm{n}=45,0,84 \pm 0,17 \mathrm{mg} / \mathrm{dl})$ und „AFLD“ ( $\mathrm{n}=16,0,87 \pm 0,15 \mathrm{mg} / \mathrm{dl}$ ) liegen diese unwesentlich, d.h. nicht-signifikant höher (Daten nicht gezeigt).

\subsubsection{Fett-/Lipidstatus}

Bezüglich des Fett-/Lipidstatus wurden die Parameter HDL, LDL und der Triglyceridspiegel erhoben (siehe Abbildung 8). Diese waren nicht Bestandteil des ursprünglichen Studienplans und wurden nicht bei allen Patienten bestimmt. Es erfolgte die retrospektive Analyse.

Die HDL unterscheidet sich nicht zwischen den untersuchten Gruppen ( $p=0,51)$. Die ermittelten Werte reichen von 46,00 \pm 18,03 mg/dl bei „AFLD“ ( $n=3)$ über die ,non-leanNAFLD“-Gruppe ( $\mathrm{n}=28$ 46,03 $\pm 11,98 \mathrm{mg} / \mathrm{dl}$ ) bis hin zu einem durchschnittlichen Wert von $72,00 \pm 41,91 \mathrm{mg} / \mathrm{dl}$ bei ,lean-NAFLD“ $(\mathrm{n}=4)$ (siehe Tabelle 8). 
High Density Lipoprotein (HDL)
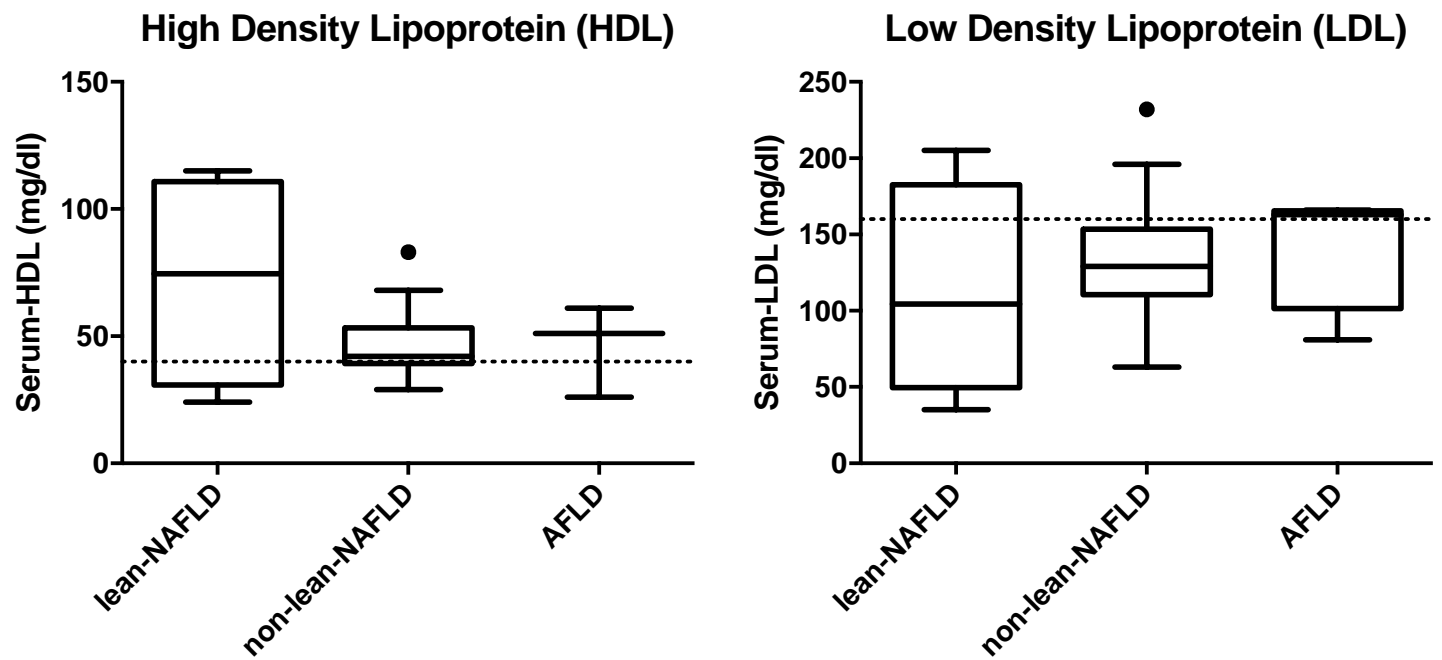

(a)

(b)

Triglyceride

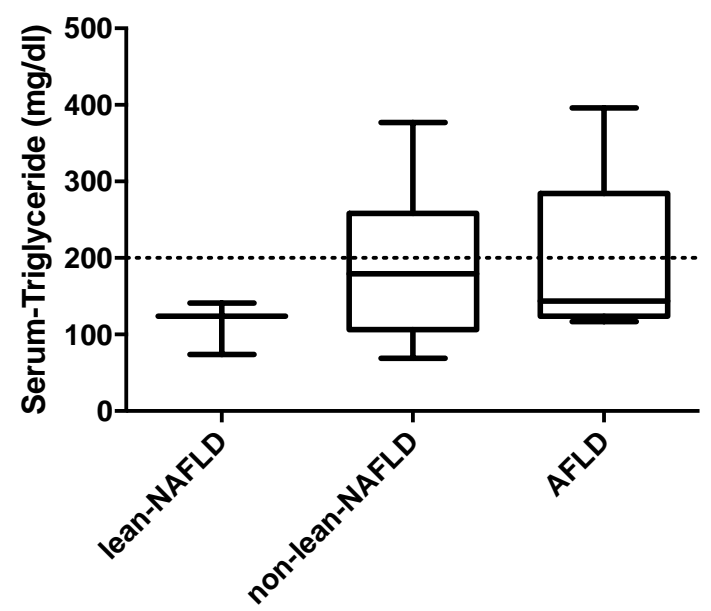

(c)

Abbildung 8: Fett-/Lipidstatus Die Graphen (a) - (c) zeigen die Parameter HDL, LDL und den Triglyceridspiegel für die angegebenen Gruppen. Die jeweiligen gültigen Referenzbereiche sind durch die gestrichelten Linien hervorgehoben.

Insgesamt unterscheiden sich HDL- $(p=0,51)$, LDL- $(p=0,44)$ und Triglyceridspiegel $(p=0,34)$ nicht.

AFLD: alcoholic fatty liver disease, HDL: high density lipoprotein, LDL: low density lipoprotein, NAFLD: non-alcoholic fatty liver disease

O. g. Beobachtung ist ebenfalls bei der Betrachtung der LDL festzustellen. Ein Unterschied liegt nicht vor $(p=0,44)$. Die niedrigsten Spiegel treten im Rahmen einer „lean-NAFLD“ ( $\mathrm{n}=4,112,3 \pm 70,59 \mathrm{mg} / \mathrm{dl}$ ), die höchsten bei „AFLD“ ( $\mathrm{n}=4,143,3 \pm 41,53 \mathrm{mg} / \mathrm{dl}$ ). 
Tabelle 8: Fett- Lipidstatus

\begin{tabular}{ccccc}
\hline \hline & lean-NAFLD & non-lean-NAFLD & AFLD & p-Wert \\
\hline Serum-HDL [mg/dl] & & & \\
(n) & 4 & 28 & 3 \\
Mean & 72,00 & 46,03 & 46,00 & 0,509 \\
Median & 74,50 & 42,00 & 51,00 & \\
Range & $24,00-115,0$ & $29,00-83,00$ & $26,00-61,00$ & \\
SD & $\pm 41,91$ & $\pm 11,98$ & $\pm 18,03$ & \\
$95 \%$ CI & $5,307-138,7$ & $41,38-50,67$ & $1,217-90,78$ \\
\hline
\end{tabular}

Serum-LDL $[\mathrm{mg} / \mathrm{dl}]$

$\begin{array}{ccccc}\text { (n) } & 4 & 29 & 4 \\ \text { Mean } & 112,3 & 133,5 & 143,3 & \\ \text { Median } & 104,5 & 129,0 & 163,0 & 0,442\end{array}$

Range $\quad 35,00-205,2 \quad 63,00-232,0 \quad 81,00-166,0$

$\begin{array}{cccc}\text { SD } & \pm 70,59 & \pm 36,07 & \pm 41,53 \\ 95 \% \text { CI } & 0-224,6 & 119,8-147,2 & 77,16-209,3\end{array}$

Serum-Triglyceride $[\mathrm{mg} / \mathrm{dl}]$

\begin{tabular}{|c|c|c|c|c|}
\hline (n) & 3 & 26 & 6 & \\
\hline Mean & 113,0 & 190,8 & 195,5 & \\
\hline Median & 124,0 & 179,5 & 143,5 & 0,338 \\
\hline Range & $74,00-141,0$ & $69,00-377,0$ & $117,0-396,0$ & \\
\hline $\mathrm{SD}$ & $\pm 34,83$ & $\pm 87,34$ & $\pm 109,0$ & \\
\hline $95 \% \mathrm{CI}$ & $26,48-199,5$ & $155,6-226,1$ & $81,08-309,9$ & \\
\hline
\end{tabular}

Aufgeführt sind die Parameter HDL, LDL und der Triglyceridspiegel

AFLD: alcoholic fatty liver disease, HDL: high density lipoprotein, LDL: low density lipoprotein, NAFLD: non-alcoholic fatty liver disease

Der Triglyceridspiegel variiert nicht zwischen den Gruppen $(p=0,34)$. Die durchschnittlich niedrigsten Werte werden bei ,lean-NAFLD“ ( $\mathrm{n}=3$ 113,0 \pm 34,83 mg/dl) beobachtet, die höchsten bei „AFLD“ ( $\mathrm{n}=6,195,5 \pm$ 109,0 mg/dl). 


\subsubsection{Suchtmittel}

Der tägliche Alkoholkonsum wurde von „AFLD“-Patienten ( $\mathrm{n}=20)$ im Durchschnitt mit $12,57 \pm 29,82 \mathrm{~g} / \mathrm{d}$ angegeben, während in der „lean-NAFLD“-Gruppe ( $\mathrm{n}=11)$ Alkoholkonsum in sämtlichen Fällen verneint wurde (siehe Abbildung 9). Im Rahmen einer „non-lean-NAFLD“ ( $\mathrm{n}=50)$ lag der durchschnittliche Tageskonsum bei 1,67 $\pm 3,50 \mathrm{~g} / \mathrm{d}$. Signifikant zeigten sich diese Unterschiede nicht $(p=0,11)$.

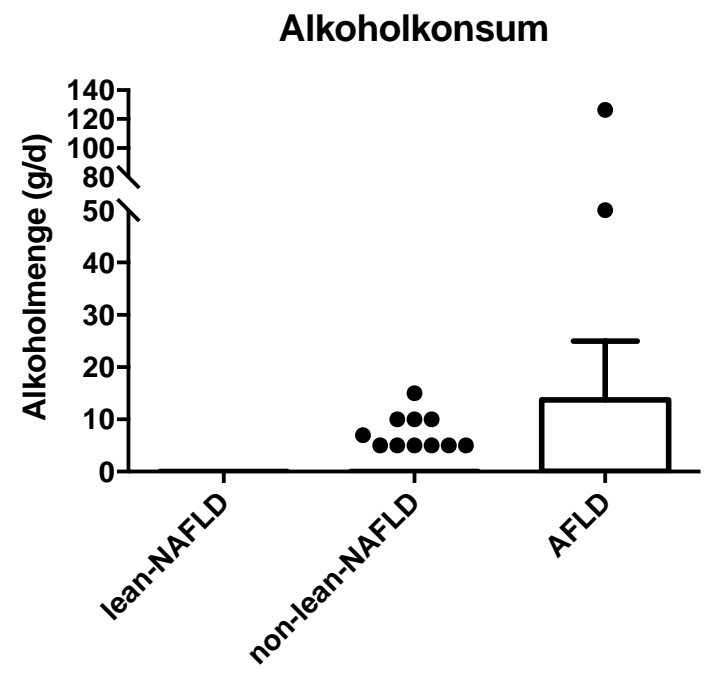

(a)

\section{Rauchverhalten}

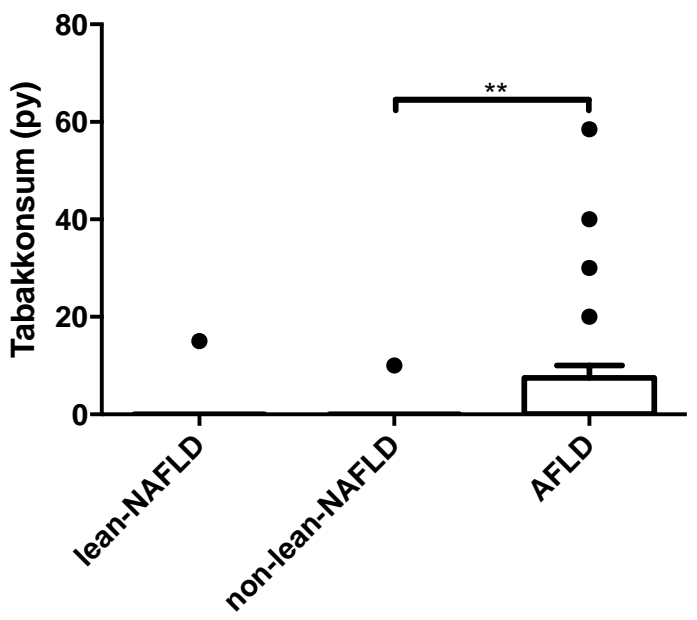

(b)

Abbildung 9: Suchtmittel Die Graphen (a) und (b) zeigen die tägliche Alkoholmenge und das Rauchverhalten (py) in den angegebenen Gruppen.

Der durchschnittliche Alkoholkonsum schwankt zwischen den Gruppen nicht $(\mathrm{p}=0,11)$. Bezüglich der Tabakbelastung gaben „AFLD“-Patienten die höchste durchschnittliche Belastung an. Sie unterschieden sich signifikant von den „non-lean-NAFLD“-Patienten ( $\mathrm{p}<0,01)$, nicht jedoch von den „lean-NAFLD“Probanden $(\mathrm{p}=0,38)$.

AFLD: alcoholic fatty liver disease, NAFLD: non-alcoholic fatty liver disease, py: pack year

Zur Einschätzung der kumulativen Tabakbelastung wurde die Bestimmung von ,pack years“ (py) vorgenommen. Insgesamt zeigen sich im Vergleich zur Normalbevölkerung (s. u.), niedrige Prävalenzen eines aktiven bzw. ehemaligen Tabakabusus in der Gesamtkohorte. Der höchste Konsum wurde von „AFLD“-Patienten ( $\mathrm{n}=12$,) mit 13,21 \pm 19,84 py angegeben. Im Vergleich dazu war die kumulative Tabakexposition bei „non-lean-NAFLD“ ( $\mathrm{n}=32$, $0,31 \pm 1,77$ py) $(\mathrm{p}<0,001)$, nicht jedoch ,lean-NAFLD“ ( $\mathrm{n}=6,2,50 \pm 6,12$ py) niedriger $(\mathrm{p}=0,38)$. 


\subsubsection{Lifestylefaktoren}

Zum Vergleich des täglichen Obstkonsums wurde der anamnestische Obstkonsum als Obstteile pro Tag (z. B. ein Apfel, eine Banane oder ein äquivalentes Volumen anderer Früchte, s. o.) analysiert. Insgesamt ergeben sich keine signifikant unterschiedlichen Obstverzehrmengen zwischen den Gruppen $(p=0,27)$ (siehe Abbildung 10). Die täglichen durchschnittlichen Verzehrmengen reichen von 1,91 \pm 1,80 Obstteile/d bei „lean-NAFLD“ ( $\mathrm{n}=11$ ), über 1,57 \pm 1,15 Obstteile/d (,non-lean-NAFLD“, $\mathrm{n}=50$ ), bis hin zu $1,10 \pm 1,11$ Obstteile/d im Rahmen einer „AFLD“ $(n=20)$.

Der tägliche Fruchtsaftkonsum unterscheidet sich nicht $(p=0,82)$. Er stellt sich wie folgt dar: „lean-NAFLD“ ( $\mathrm{n}=11)$ mit 127,3 \pm 300,3 ml/d, „,non-lean-NAFLD“ ( $\mathrm{n}=50)$ mit 66,10 \pm 140,6 ml/d und ,AFLD“ ( $\mathrm{n}=20) \mathrm{mit} 212,5 \pm$ 460,8 ml/d.

Der tägliche Softdrink-Konsum ist im untersuchten Kollektiv kein Differenzierungsmerkmal $(p=0,13)$. Durchschnittlich rangieren die täglichen Verzehrmengen zwischen $112,5 \pm 503,1 \mathrm{ml} / \mathrm{d}$ bei „AFLD“ ( $\mathrm{n}=20), 130,0 \pm 216,3 \mathrm{ml} / \mathrm{d}$ bei ,lean-NAFLD“ ( $\mathrm{n}=11)$ und 171,6 $\pm 368,9 \mathrm{ml} / \mathrm{d}$ im Rahmen einer „,non-lean-NAFLD“ $(\mathrm{n}=50)$.

Die „wöchentliche sportliche Betätigung“ ist nicht identisch unter den o. g. Gruppen $(\mathrm{p}=0,02)$. Sie ist bei „non-lean-NAFLD“ ( $\mathrm{n}=50,1,94 \pm 2,46 \mathrm{~d} / \mathrm{w})$ am höchsten. Im Vergleich dazu ist das wöchentliche Ausmaß sportlicher Aktivität der „AFLD“-Patienten ( $\mathrm{n}=20)$ mit $0,50 \pm 1,67 \mathrm{~d} / \mathrm{w}$ deutlich niedriger $(\mathrm{p}=0,01)$. „Lean-NAFLD“-Probanden $(\mathrm{n}=11,1,64 \pm 2,77 \mathrm{~d} / \mathrm{w})$ gruppieren sich zwischen den vorgenannten Gruppen ein.

Keine Unterschiede bestehen bezüglich des täglichen Kaffeekonsums ( $p=0,16)$. Mit 1,64 \pm 2,42 Tassen/d (,lean-NAFLD“, $\mathrm{n}=11), 2,02 \pm$ 1,61 Tassen/d (,non-lean-NAFLD“, $\mathrm{n}=50)$ und 1,38 \pm 1,60 Tassen/d (,AFLD“, $\mathrm{n}=20$ ) liegen identische Werte vor. 

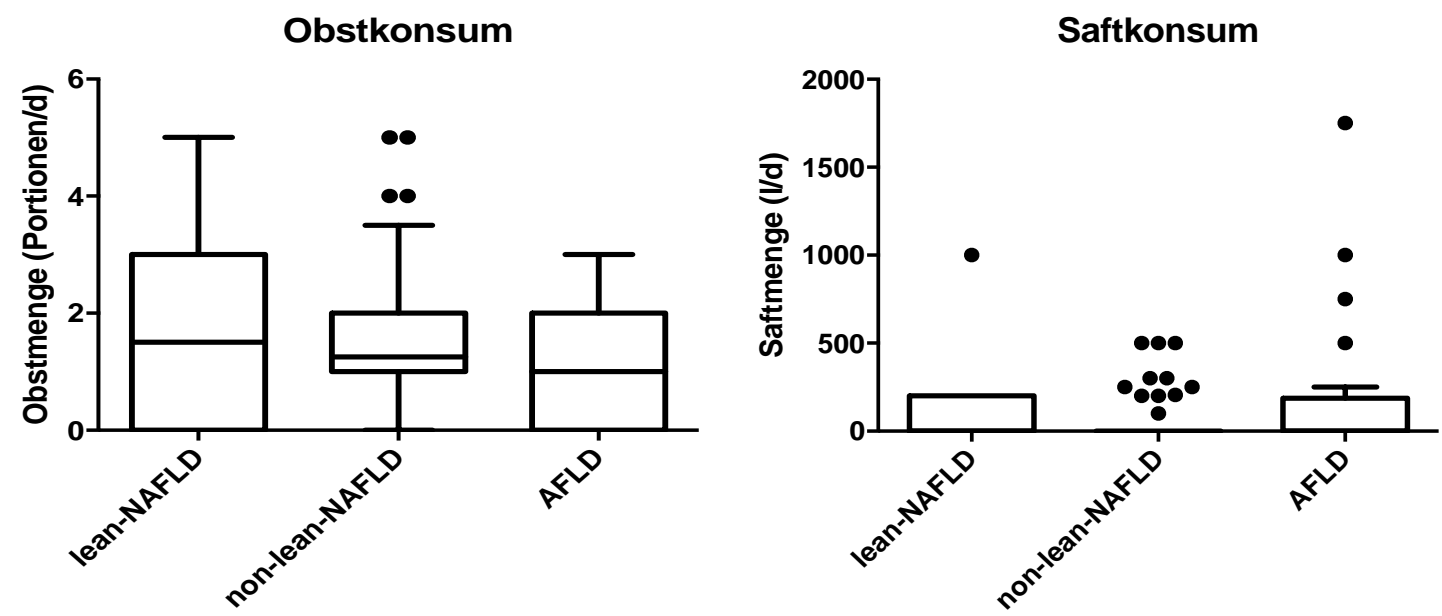

(a)

(b)

Softdrinkkonsum
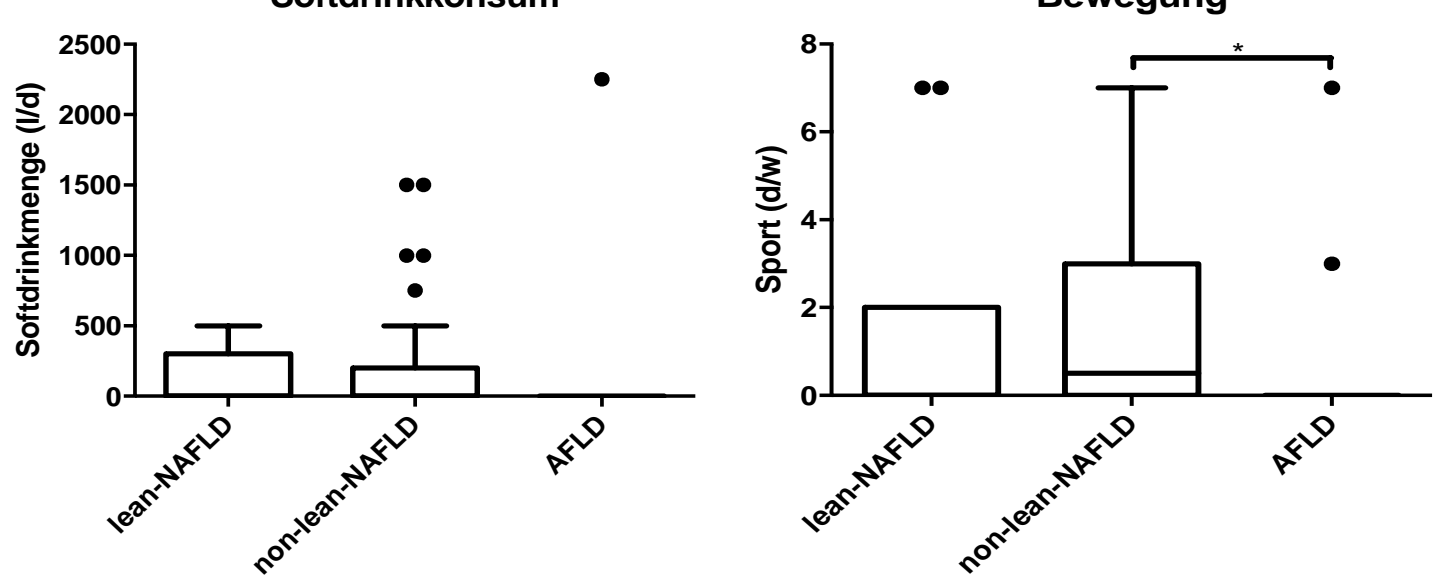

(c)

(d)

Kaffeekonsum

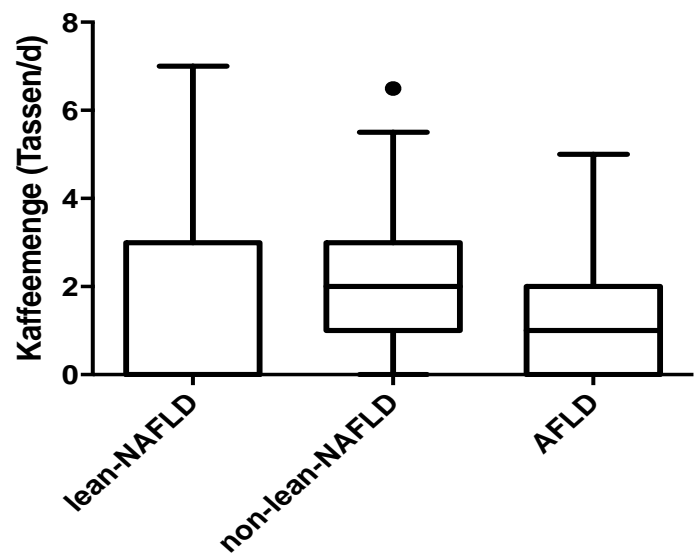

(e)

Abbildung 10: Lifestylefaktoren Die Graphen (a) - (e) zeigen den Obst-, Saft- und Softdrinkkonsum sowie die wöchentliche Häufigkeit sportlicher Aktivität und den Kaffeekonsum in den angegebenen Gruppen.

Signifikant unterschiedliche Lifestylegewohnheiten bestehen lediglich zwischen den „non-lean-NAFLD“- und „AFLD“-Patienten hinsichtlich der sportlichen Aktivität.

AFLD: alcoholic fatty liver disease, NAFLD: non-alcoholic fatty liver disease 


\subsection{Kurzzeit-Follow-up der lean-NAFLD im Vergleich zur non-lean- NAFLD}

Von den insgesamt 61 NAFLD-Patienten stellten sich 50 Patienten (81,97 \%) im Follow-upIntervall erneut vor. Die Wiedervorstellung erfolgte im Durchschnitt nach $165,1 \pm 78,72$ Tagen. Bei elf Patienten (18,03\%) erfolgte bis zum Ende des Beobachtungszeitraumes (31.12.2017) keine erneute Wiedervorstellung, sodass der Ausschluss aus der Follow-up-Analyse erfolgte. Im Rahmen der WV wurde erneut das Körpergewicht gemessen, der BMI bestimmt und laborchemisch unteranderem die AST, ALT, gGT und AP erhoben. Es erfolgte ein Vergleich der absoluten Mittelwerte bei Erstkontakt und Wiedervorstellung sowie ein Vergleich der absoluten Unterschiede $(\Delta)$ die zwischen EK und WV vorlagen.

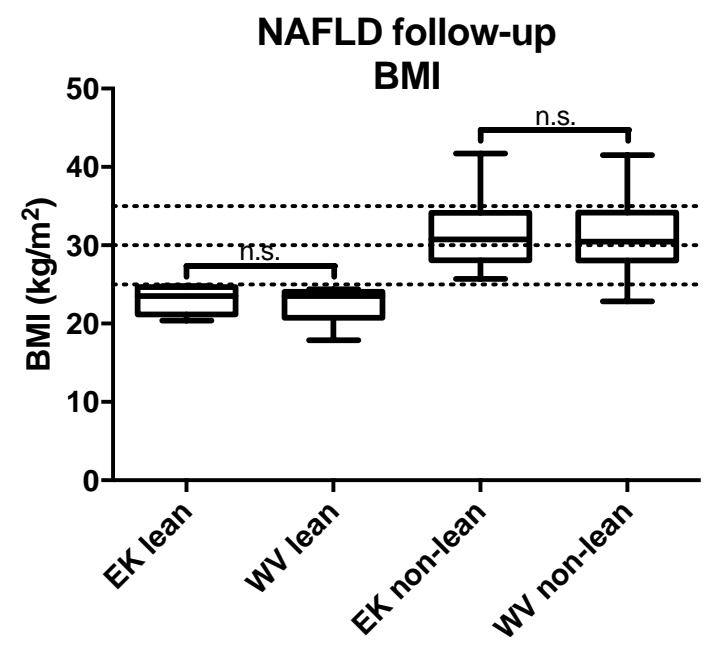

(a)

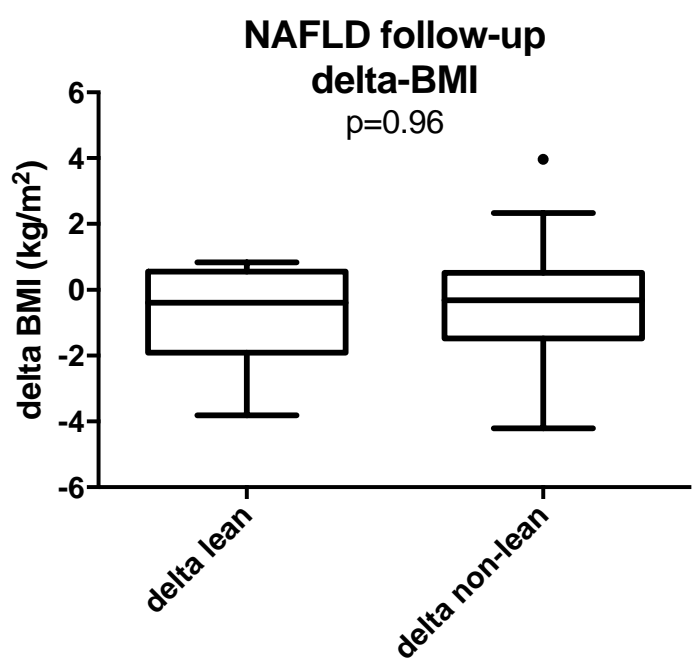

(b)

Abbildung 11: Entwicklung des BMI im Follow-up-Intervall Die Abbildungen (a) und (b) zeigen die Parameter BMI und $\Delta$ BMI bzw. deren korrespondierende, durchschnittliche Spiegel bei EK und WV. Die jeweiligen Referenzbereiche sind durch die gestrichelten Linien hervorgehoben.

Die Veränderung des BMI zwischen EK und WV ist weder in den Subgruppen ( $p=0,45$ bzw. $p=0,50)$ noch das $\Delta$ BMI betreffend signifikant $(\mathrm{p}=0,96)$.

BMI: body mass index, EK: Erstkontakt, NAFLD: non-alcoholic fatty liver disease, WV: Wiedervorstellung

51 NAFLD-Patienten wurden im Follow-up-Intervall bezüglich ihres BMI reevaluiert (siehe Abbildung 11). Im Mittel änderte sich der BMI sowohl bei „lean-NAFLD“-Patienten $\left(\mathrm{n}=9,-0,71 \pm 1,58 \mathrm{~kg} / \mathrm{m}^{2}\right)(\mathrm{p}=0,45)$, als ,non-lean-NAFLD“-Probanden $(\mathrm{n}=41$, $\left.-0,55 \pm 1,83 \mathrm{~kg} / \mathrm{m}^{2}\right)(\mathrm{p}=0,50)$ nicht zwischen EK und WV. Das $\Delta$ BMI unterscheidet sich ebenfalls nicht zwischen den vorgenannten Gruppen $(\mathrm{p}=0,96)$ (siehe Tabelle 9). 
Tabelle 9: Absolute Veränderung ausgewählter Parameter im Follow-up-Intervall

\begin{tabular}{|c|c|c|c|}
\hline lean-NAFLD & non-lean-NAFLD & AFLD & p-Wert \\
\hline (n) & 9 & 41 & \\
\hline \multicolumn{4}{|l|}{$\Delta \mathrm{BMI}\left[\mathrm{kg} / \mathrm{m}^{2}\right]$} \\
\hline Mean & $-0,71$ & $-0,55$ & \multirow{6}{*}{0,960} \\
\hline Median & $-0,40$ & $-0,32$ & \\
\hline Range & $-3,82-0,83$ & $-4,20-3,96$ & \\
\hline SD & $\pm 1,58$ & $\pm 1,83$ & \\
\hline $95 \% \mathrm{CI}$ & $-1,93-0,51$ & $-1,13-0,028$ & \\
\hline p-Wert (EK vs. WV) & 0,4489 & 0,5019 & \\
\hline \multicolumn{4}{|l|}{$\Delta$ AST $[\mathrm{U} / 1]$} \\
\hline Mean & $-1,00$ & $-12,93$ & \multirow{6}{*}{0,809} \\
\hline Median & $-6,00$ & $-4,00$ & \\
\hline Range & $-27,00-59,00$ & $-168,00-41,00$ & \\
\hline SD & $\pm 24,44$ & $\pm 34,16$ & \\
\hline $95 \% \mathrm{CI}$ & $-19,79-17,79$ & $-23,71-(-2,15)$ & \\
\hline p-Wert (EK vs. WV) & 0,0890 & 0,0387 & \\
\hline \multicolumn{4}{|l|}{$\Delta$ ALT $[\mathrm{U} / \mathrm{l}]$} \\
\hline Mean & $-6,33$ & $-33,07$ & \multirow{6}{*}{0,809} \\
\hline Median & $-12,00$ & $-9,00$ & \\
\hline Range & $-65,00-67,00$ & $-583,00-50,00$ & \\
\hline SD & $\pm 40,54$ & $\pm 97,31$ & \\
\hline $95 \% \mathrm{CI}$ & $-37,50-24,83$ & $-63,79-(-2,36)$ & \\
\hline p-Wert (EK vs. WV) & 0,1977 & 0,0096 & \\
\hline \multicolumn{4}{|l|}{$\Delta \mathrm{gGT}[\mathrm{U} / \mathrm{l}]$} \\
\hline Mean & 40,89 & $-50,07$ & \multirow{6}{*}{0,353} \\
\hline Median & $-5,000$ & $-10,00$ & \\
\hline Range & $-35,00-255,0$ & $-983,0-117,0$ & \\
\hline SD & $\pm 107,7$ & $\pm 167,8$ & \\
\hline $95 \% \mathrm{CI}$ & $-41,87-123,6$ & $-103,0-2,882$ & \\
\hline p-Wert (EK vs. WV) & 0,9314 & 0,2526 & \\
\hline
\end{tabular}




\begin{tabular}{lccc} 
lean-NAFLD & non-lean-NAFLD & AFLD & p-Wert \\
\hline $\mathbf{n})$ & $\mathbf{9}$ & $\mathbf{4 1}$ & \\
\hline $\boldsymbol{\Delta}$ AP $[\mathbf{U} / \mathbf{1}]$ & 14,22 & $-15,98$ & \\
Mean & 11,00 & $-9,000$ & $\mathbf{0 , 0 0 9}$ \\
Median & $-18,00-73,00$ & $-278,0-28,00$ & \\
Range & $\pm 26,65$ & $\pm 46,03$ & \\
SD & $-6,26-34,71$ & $-30,51-(-1,45)$ & \\
$95 \%$ CI & 0,5882 & 0,1247 & \\
p-Wert (EK vs. WV) & & \\
\hline \hline
\end{tabular}

ALT: Alanin-Aminotransferase, AST, Aspartat-Aminotransferase, BMI: body mass index, EK: Erstkontakt, gGT: Gamma-Glutamyltransferase, NAFLD: non-alcoholic fatty liver disease, WV: Wiedervorstellung

Der AST-Spiegel ist sowohl zum Zeitpunkt des EK ( $p=0,56)$, als auch bei WV $(p=0,40)$ nicht unterschiedlich hoch in den NAFLD-Subgruppen. Das $\triangle$ AST $(\mathrm{p}=0,81)$ ist ebenfalls identisch (siehe Abbildung 12). In der „lean-NAFLD“-Gruppe ( $\mathrm{n}=9,1,00 \pm 24,44 \mathrm{U} / 1$ bleibt die AST stabil $(p=0,09)$, während bei Patienten mit „,non-lean-NAFLD“ $(n=41)$ ein Rückgang von $12,93 \pm 34,16 \mathrm{U} / 1$ zu verzeichnen ist $(\mathrm{p}=0,04)$.

Die durchschnittliche ALT ist sowohl bei EK $(p=0,42)$, als auch bei WV ( $p=0,66)$ gleich hoch in den Vergleichsgruppen. Es ist ebenfalls kein Unterschied bezüglich der $\Delta$ ALT $(\mathrm{p}=0,81)$ festzustellen. In der „lean-NAFLD“-Gruppe $(\mathrm{n}=9)$ bleibt die ALT mit einer $\Delta$ ALT von $-6,33 \pm 40,54 \mathrm{U} / 1$ konstant $(\mathrm{p}=0,20)$. Bei Patienten mit ,non-lean“-NAFLD ( $\mathrm{n}=41)$ fällt dieser Wert um 33,07 \pm 97,31 U/1 ( $<<0,01)$. 

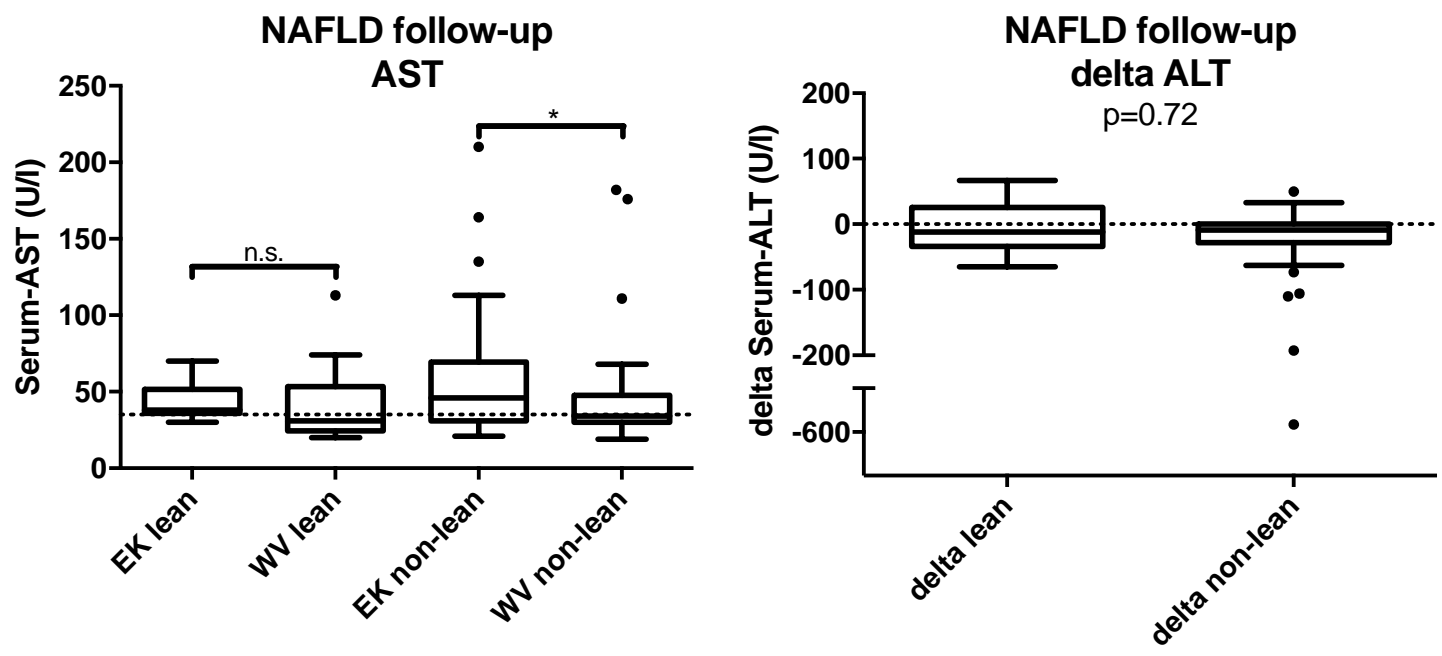

(a)

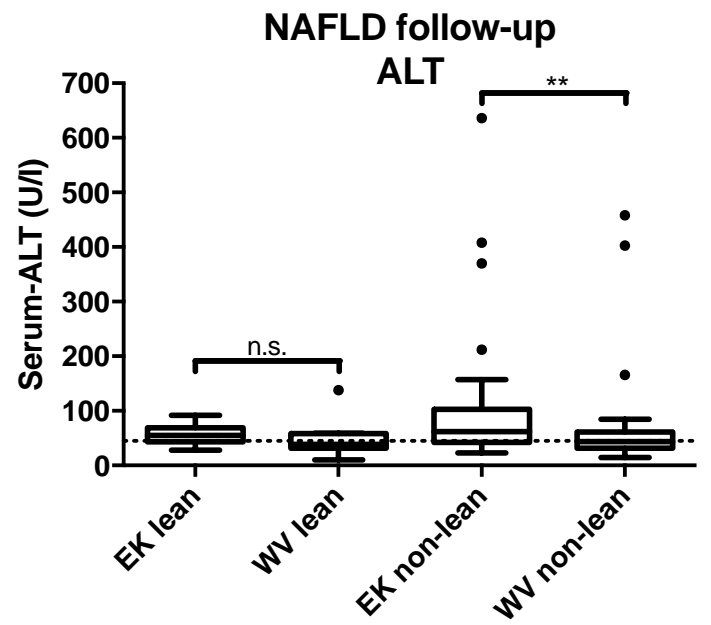

(c)

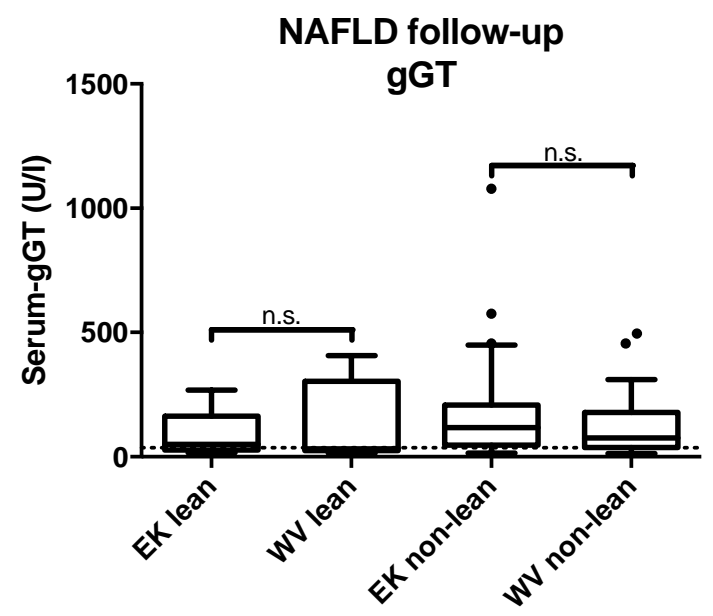

(b)

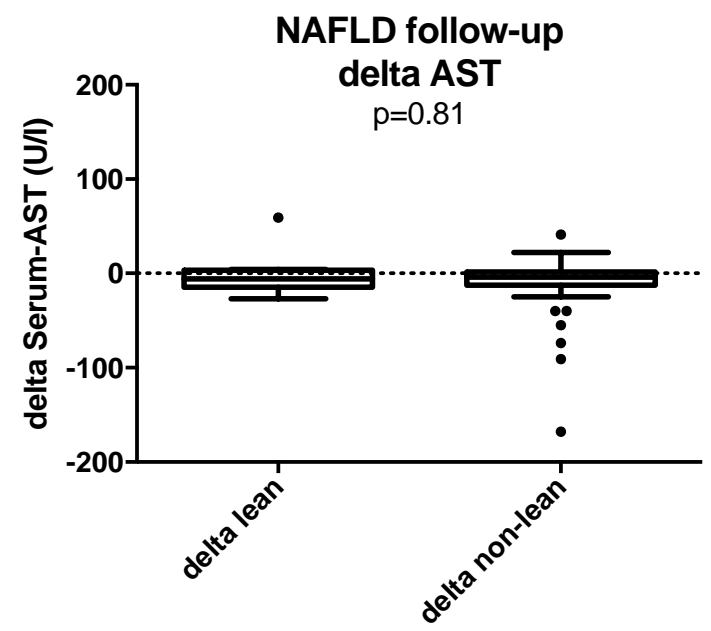

(d)

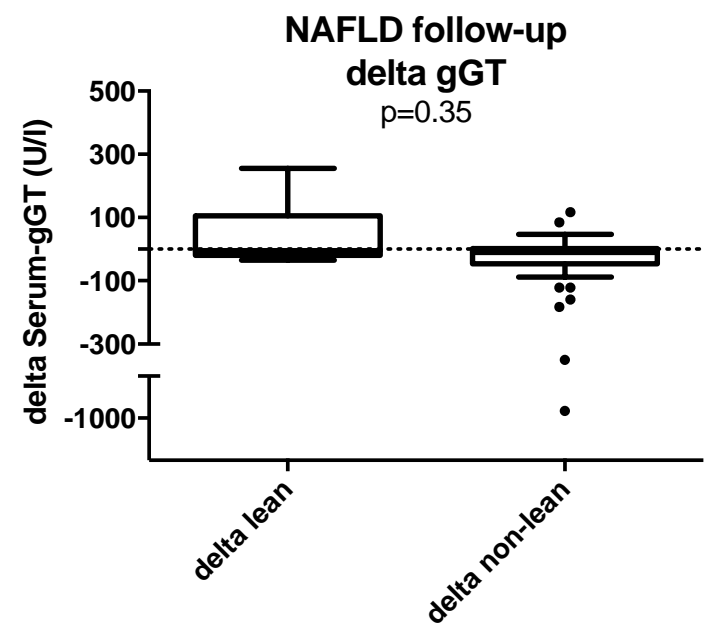

(e) 


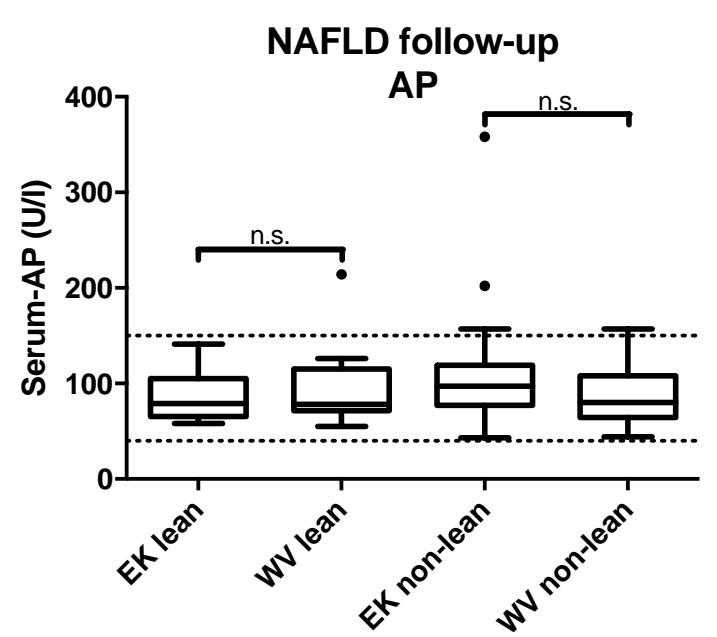

(g)

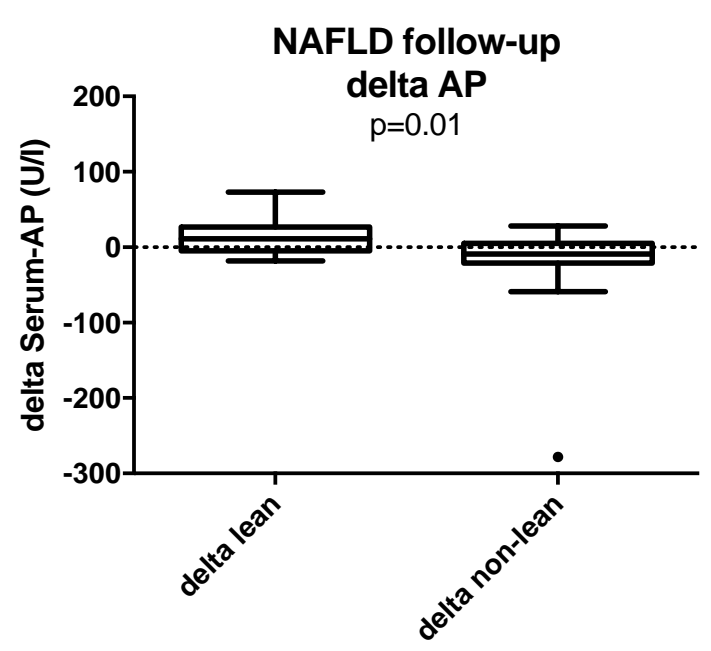

(h)

Abbildung 12: Absolute Veränderung ausgewählter Laborparameter im Follow-up-Intervall Die Abbildungen (a) - (h) zeigen die durchschnittliche absolute Veränderung der Parameter AST, $\Delta$ AST, ALT, $\Delta$ ALT, gGT, $\Delta$ gGT und AP, $\Delta$ AP bzw. deren korrespondierende, durchschnittliche Spiegel bei EK und Wiedervorstellung WV in den angegebenen Gruppen. Die jeweiligen gültigen Referenzbereiche sind durch die gestrichelten Linien hervorgehoben.

Die Veränderung der o. $\mathrm{g}$. Parameter $(\Delta$ AST: $\mathrm{p}=0,81, \Delta$ ALT: $\mathrm{p}=0,72$ und $\Delta$ gGT: $\mathrm{p}=0,35)$ variiert nicht zwischen den „NAFLD“-Subgruppen. Lediglich im Vergleich der $\Delta$ AP ist eine signifikante Differenz feststellbar $(p=0,01)$.

ALT: Alanin-Aminotransferase, AP: alkalische Phosphatase, AST: Aspartat-Aminotransferase, EK: Erstkontakt, gGT: Gamma-Glutamyltransferase, NAFLD: non-alcoholic fatty liver disease, WV: Wiedervorstellung

Die gGT ist zum Zeitpunkt des EK ( $p=0,26)$ und der WV $(p=0,60)$ gleich hoch in beiden NAFLD-Subgruppenvergleich. Bezüglich des $\Delta \mathrm{gGT}(\mathrm{p}=0,35)$ ist ebenfalls kein Unterschied zu dokumentieren. Bezüglich der Höhe des gGT-Spiegels bleiben sowohl die „lean-NAFLD“- (n = 9, $\Delta$ gGT: 40,89 \pm 107,7 U/l) (p = 0,93), als auch „non-lean-NAFLD“Patienten (n $=41, \Delta \mathrm{gGT}:-50,07 \pm 167,8 \mathrm{U} / \mathrm{L})(\mathrm{p}=0,25)$ konstant.

Weder die Höhe der AP bei EK ( $p=0,24)$, noch bei WV ( $p=0,79)$ variieren in signifikantem Ausmaß innerhalb der „NAFLD“-Subgruppen. Bezüglich der $\Delta$ AP konnte eine unterschiedliche Entwicklung festgestellt werden $(\mathrm{p}<0,01)$. Liegt eine „lean-NAFLD“ $(\mathrm{n}=9)$ vor, bleibt die AP mit einer $\Delta$ AP von 14,22 $\pm 26,65 \mathrm{U} / 1$ konstant $(\mathrm{p}=0,59)$, bei einer „non-lean-NAFLD“ $(\mathrm{n}=41)$ beträgt die, $\Delta$ AP $-15,98 \pm 46,03 \mathrm{U} / 1(\mathrm{p}=0,12)$. 


\subsection{Effekte einer standardisierten Kurzberatung im Kurzzeitintervall}

Von den 50 Patienten des NAFLD-Kollektivs, die sich im Follow-up wiedervorstellten, lagen bei sämtlichen Patienten Daten zur Körpergewichtsentwicklung vor. Das Körpergewicht wurde bei EK und WV im LZG gemessen und der jeweilige, korrespondierende BMI berechnet. Im Folgenden konnten die Patienten der Gruppe „Gewichtsverlust“ bzw. „kein Gewichtsverlust“" zugeordnet werden.
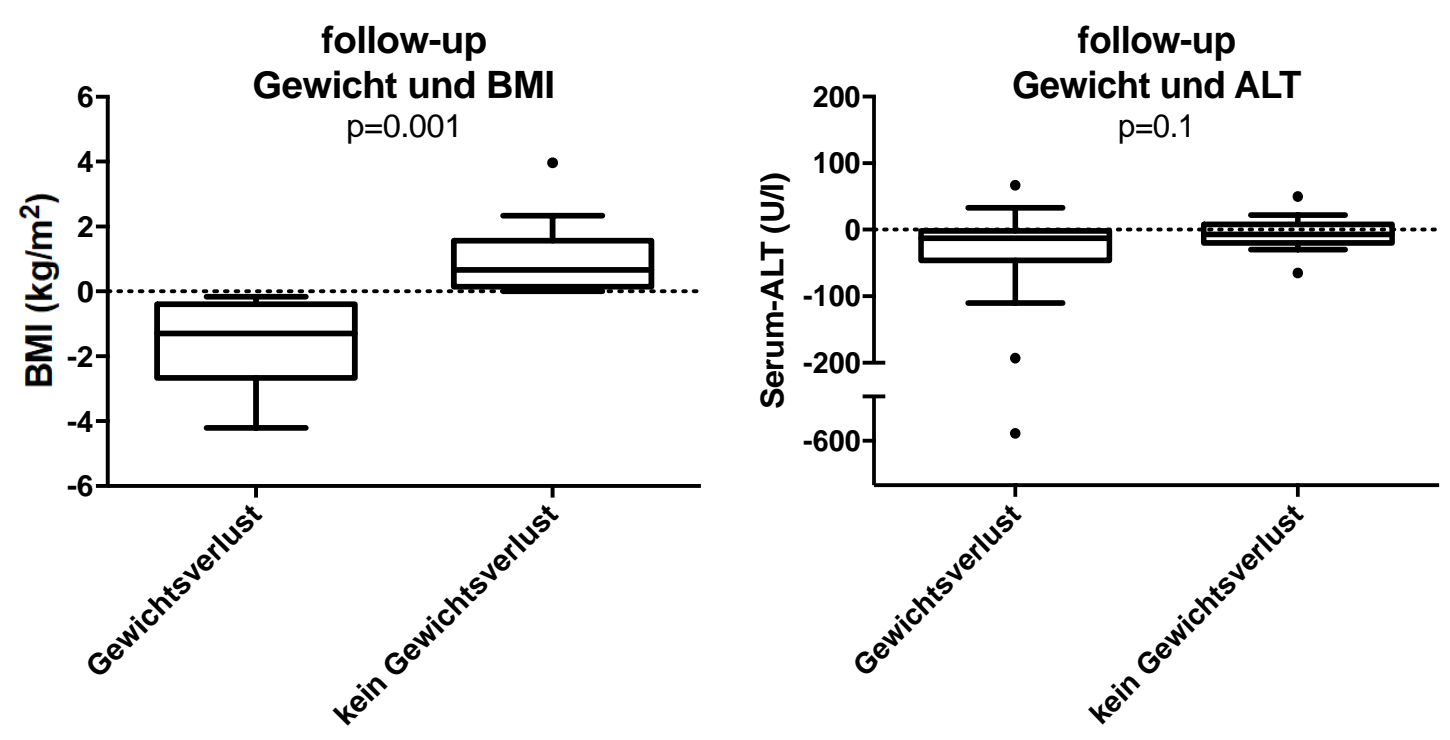

(a)

(b)

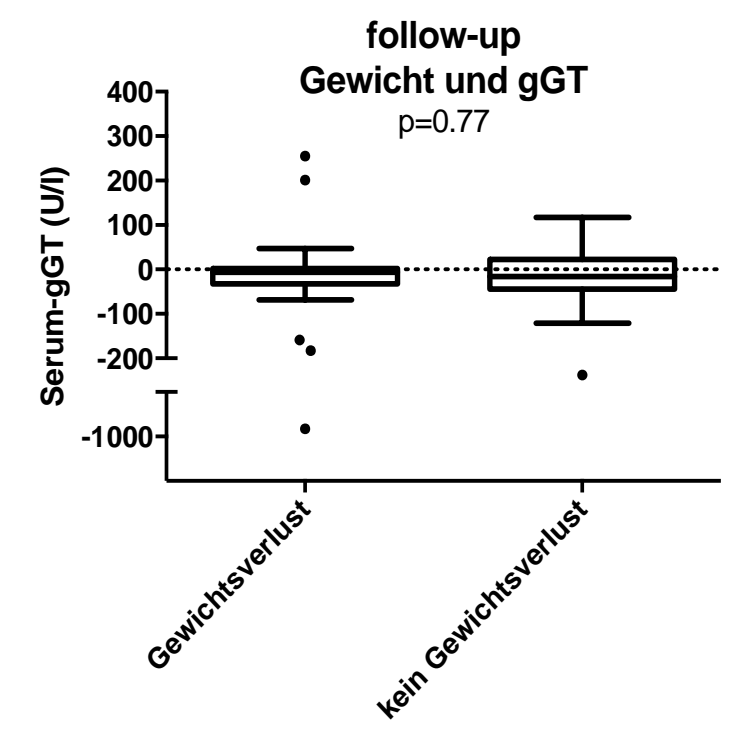

(c)

Abbildung 13: "Gewichtsverlust" vs. "kein Gewichtsverlust" Die Graphen (a) - (c) zeigen die Parameter $\Delta \mathrm{BMI}, \Delta \mathrm{ALT}$ und $\Delta \mathrm{gGT}$ für die angegebenen Gruppen. Die jeweiligen gültigen Referenzbereiche sind durch die gestrichelten Linien hervorgehoben.

Das $\Delta$ BMI ist signifikant unterschiedlich zwischen den Gruppen „Gewichtsverlust“ vs. ,kein Gewichtsverlust“ $(\mathrm{p}<0,001)$. Die $\Delta$ ALT $(\mathrm{p}=0,10)$ und die $\Delta$ gGT unterscheiden sich hingegen nicht $(\mathrm{p}=0,77)$. 
29 von 50 „NAFLD“-Patienten (58 \%) reduzierten innerhalb des Follow-Up-Intervalls ihren BMI („Gewichtsverlust“). Die durchschnittliche Reduktion lag bei 1,67 \pm 1,30 BMI-Punkten (30,34 \pm 5,07 BMI-Punkte auf 28,67 \pm 5,15 BMI-Punkte, bzw. - 5,50 \% des Körpergewichts) ( $\mathrm{p}<0,0001)$. Bei 21 Patienten (42\%) erfolgte keine Veränderung bzw. ein Anstieg des BMI (,kein Gewichtsverlust“). Der durchschnittliche BMI stieg im Vergleich zum EK in dieser Gruppe um 0,93 \pm 0,98 BMI-Punkte $(29,84 \pm$ 5,06 BMI-Punkte auf 30,78 \pm 5,47 BMIPunkte, bzw. $+3,15 \%$ des Körpergewichts) $(\mathrm{p}<0,001)$. Formal ist der durchschnittliche BMI beider Gruppen sowohl bei EK ( $p=0,87)$, als auch bei WV ( $p=0,15)$ identisch. Das $\Delta$ BMI hingegen ist definitionsgemäß hochsignifikant unterschiedlich $(\mathrm{p}<0,001)$ (siehe Abbildung 13).

Die ALT war stabil in der Gruppe „kein Gewichtsverlust“ ( $\mathrm{n}=21$, $\Delta$ ALT: $-5,43 \pm 23,49 \mathrm{U} / 1,78,81 \pm 79,66 \mathrm{U} / 1$ auf 73,38 \pm 93,23 U/l) (p = 0,24). Bei den „Gewichtsverlust“-Patienten ( $\mathrm{n}=29, \Delta$ ALT: $-44,79 \pm 114,5 \mathrm{U} / 1,101,8 \pm 125,2 \mathrm{U} / 1$ auf 56,97 U/l) kam es zu einem signifikanten Rückgang der ALT ( $<<0,01)$ (siehe Tabelle 10).

Die „Gewichtsverlust“-Gruppe $(\mathrm{n}=29)$ weist im Durchschnitt eine Reduktion der gGT von $39,83 \pm 198,2 \mathrm{U} / 1(138,3 \pm 206,7 \mathrm{U} / 1$ auf 98,52 \pm 123,3 U/l) $(\mathrm{p}=0,02)$ auf. Erfolgt „,kein

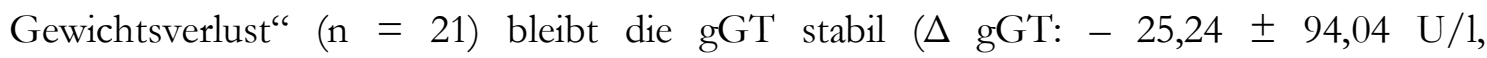
$175,8 \pm 142,9 \mathrm{U} / \mathrm{l}$ auf 150,5 $\pm 114,3 \mathrm{U} / \mathrm{l})(\mathrm{p}=0,34)$. Signifikante Unterschiede in der Höhe des gGT-Spiegels liegen nicht bei EK $(p=0,08)$, jedoch bei WV $(p=0,03)$ vor. Dies trifft nicht auf die $\Delta \mathrm{gGT} z u(\mathrm{p}=0,77)$. 
Tabelle 10: „Gewichtsverlust“ vs. „,kein Gewichtsverlust“

\begin{tabular}{|c|c|c|c|}
\hline & Gewichtsverlust & Kein Gewichtsverlust & p-Wert \\
\hline (n) & 29 & 21 & \\
\hline \multicolumn{4}{|l|}{$\Delta \mathrm{BMI}\left[\mathrm{kg} / \mathrm{m}^{2}\right]$} \\
\hline Mean & $-1,67$ & 0,93 & \\
\hline Median & $-1,30$ & 0,66 & \\
\hline Range & $-4,20-(-0,17)$ & $0,0-3,96$ & 0,001 \\
\hline $\mathrm{SD}$ & $\pm 1,37$ & $\pm 0,98$ & \\
\hline $95 \% \mathrm{CI}$ & $-2,19-(-1,153)$ & $0,49-1,38$ & \\
\hline p-Wert (EK vs. WV) & $<0,0001$ & 0,0005 & \\
\hline \multicolumn{4}{|l|}{$\Delta \mathrm{ALT}[\mathrm{U} / 1]$} \\
\hline Mean & $-44,79$ & $-5,429$ & \\
\hline Median & $-13,00$ & $-7,000$ & \\
\hline Range & $-583,0-67,00$ & $-65,00-50,00$ & 0,096 \\
\hline SD & $\pm 114,5$ & $\pm 23,49$ & \\
\hline $95 \% \mathrm{CI}$ & $-88,36-(-1,226)$ & $-16,12-5,266$ & \\
\hline p-Wert (EK vs. WV) & 0,0015 & 0,2395 & \\
\hline \multicolumn{4}{|l|}{$\Delta \mathrm{gGT}(\mathrm{U} / 1)$} \\
\hline Mean & $-39,83$ & $-25,24$ & \\
\hline Median & $-6,000$ & $-16,00$ & \\
\hline Range & $-983,0-255,0$ & $-349,0-117,0$ & 0,774 \\
\hline $\mathrm{SD}$ & $\pm 198,2$ & $\pm 94,04$ & \\
\hline $95 \% \mathrm{CI}$ & $-115,2-35,57$ & $-68,05-17,57$ & \\
\hline p-Wert (EK vs. WV) & 0,0178 & 0,3409 & \\
\hline
\end{tabular}

Aufgeführt sind die Parameter $\Delta$ BMI, $\Delta$ ALT und $\Delta$ gGT für die angegebenen Gruppen.

ALT; Alanin-Aminotransferase, BMI: body mass index, EK: Erstkontakt, gGT: Gamma-Glutamyltransferase, WV: Wiedervorstellung 


\section{Diskussion}

\subsection{Kollektivübersicht}

Im untersuchten Kollektiv liegt der Anteil der NAFLD-Patienten bei 36,31 \% und stellt noch vor viralen Hepatitiden (18,45 \%) und der AFLD (11,90\%) die häufigste Entität dar. Dies deckt sich mit publizierten Beobachtungen (Weiss et al. 2014; Roeb et al. 2015; Schwimmer et al. 2006). Die Häufigkeit der Diagnose „NAFLD“ und ihrer Komplikationen ist deutlich ansteigend, nicht zuletzt bedingt durch ein erhöhtes Problembewusstsein basierend auf aktuellen Forschungsdaten und einer sukzessiv entstandenen deutschen Leitlinie (Roeb 2015; Alexander et al. 2018; Welsh et al. 2013; Weiss et al. 2014; Estes et al. 2017; Kabbany et al. 2017). Auch im untersuchten (vorselektionierten) Kollektiv einer hepatologisch spezialisierten Hochschulambulanz zeigen sich somit hohe NAFLD-Prävalenzen.

Zunehmende Beachtung erfährt im letzten Jahrzehnt zudem die lean-NAFLD, deren Anteil im untersuchten Kollektiv bei 18,03 \% liegt. Diese Größenordnung deckt sich mit zahlreichen epidemiologischen Studien, die einen Anteil von lean-Patienten von $7-19 \%$ angeben (Yoshitaka et al. 2017; Margariti et al. 2013; Wei et al. 2015; Hagström et al. 2018; Akyuz et al. 2015; Cohen et al. 2011; Younossi et al. 2012). Die lean-NAFLD stellt somit eine relevante, weil häufige, Entität dar. Unklar bleibt jedoch, ob die lean-NAFLD vergleichbare Risiken oder ein ggf. abweichendes Progressionsverhalten zeigt (Hagström et al. 2018).

\subsection{Differenzierbarkeit von AFLD und NAFLD anhand klinischer Routineparameter}

\subsubsection{Epidemiologische und anthropometrische Charakteristika des Kollektivs}

Das Patientenalter ist im Studienkollektiv zwischen AFLD und non-lean-NAFLD vergleichbar. Lean-NAFLD-Patienten hingegen sind im Gegensatz dazu signifikant jünger. Im wissenschaftlichen Diskurs wird das Lebensalter kontrovers bewertet. Insgesamt scheint mit Ausnahme weniger Arbeiten (Anurag et al. 2015; Gonzalez-Cantero et al. 2018) ein Zusammenhang zwischen NAFLD und höherem Lebensalter vorzuliegen (Kagansky et al. 2004; Chen et al. 2007; Ong et al. 2008; Hagström et al. 2018; Feng et al. 2017; Xu et al. 2013; Alexander et al. 2018). Die lean-NAFLD gilt allgemein eher mit einem niedrigeren Lebensalter vergesellschaftet (Younossi et al. 2012; Feng et al. 2014; Naderian et al. 2017; Sookoian und Pirola 2017; Feng et al. 2017). Die lean-NAFLD-Gruppe des untersuchten Kollektivs konnte diesen Sachverhalt ebenfalls abbilden.

Das Geschlechterverhältnis ist in dieser Studie, wie auch in anderen Studien gezeigt, ausgeglichen (Gonzalez-Cantero et al. 2018; Alexander et al. 2018). In verschiedenen 
Untersuchungen zeigten sich z. T. erhöhte NAFLD-Prävalenzen beim männlichen Geschlecht, bzw. eine Bevorzugung des weiblichen Geschlechts im Rahmen einer NASH (Roeb et al. 2015; Hagström et al. 2018). Bei lean-NAFLD wird insgesamt ein ausgeglichenes Geschlechterverhältnis bzw. Bevorzugung des weiblichen Geschlechts angenommen (Younossi et al. 2012; Feng et al. 2014).

Die Blutdruckparameter der Studienpatienten waren insgesamt normwertig und unterschieden sich analog zu weiteren Studienergebnissen nicht zwischen den untersuchten Gruppen (Feng et al. 2017). Lediglich im NAFLD-Kollektiv konnte mit Hinweis auf die Studie Kims et al. ein nicht-signifikanter RR-Anstieg bei Zunahme des BMI beobachtet werden (Kim et al. 2004). Limitierend ist hier vor allem die Tatsache, dass ein Teil der übergewichtigen/fettleibigen Patienten dieser Studie sich bereits unter antihypertensiver Therapie befand. Eine (therapeutisch beabsichtigte) Beeinflussung ggf. erhöhter Blutdruckparameter muss bei diesen Patienten unterstellt werden.

\subsubsection{Blutbild}

Die Blutbildparameter der Studiengruppen liegen im Normbereich und zeigen mit Ausnahme der Erythrozytenzahl, des MCV und der Thrombozytenzahl keine Unterschiede zwischen den Gruppen. Insgesamt ist die Studienlage in diesem Kontext durch konträre Ergebnisse gekennzeichnet (Feng et al. 2014; Hagström et al. 2018; Xu et al. 2013; Bernhardt et al. 2018). Allgemeingültige Aussagen bzw. pathophysiologische Erklärungsansätze fehlen z. T., sodass z. B. die Beobachtung bzw. Bedeutung unterschiedlicher Erythrozytenzahlen der NAFLD- und AFLD-Gruppe unklar bleiben.

Diskutiert werden muss das MCV, welches Folsäuremangel-bedingt bei AFLD häufig erhöht ist und im Rahmen einer hepatologischen Erstabklärung häufig differenzialdiagnostisch genutzt wird. Im Vergleich mit der NAFLD war diese Differenzierung im Kollektiv nicht gegeben. Einen Erklärungsansatz bildet ggf. der hohe Anteil abstinenter AFLD-Patienten im Rahmen dieser Studie. Erfahrungsgemäß nehmen aktive Alkoholtrinker, welche häufig durch ein erhöhtes MCV auffallen, die Termine in der Ambulanz seltener wahr.

Die Thrombozytenzahl bei AFLD fiel im Kollektiv deutlich niedriger als bei NAFLD aus. Pathophysiologisch ist dies am ehesten durch vermehrten intrasplenischen Abbau bei Pfortaderhochdruck und konsekutiver Splenomegalie zu erklären. Die untersuchten AFLDPatienten waren in der Regel bereits in einer fortgeschrittenen Phase der Lebererkrankung (MELD-Score: 10,00 \pm 4,20). Im Kontrast dazu lagen bei den NAFLD-Patienten dieses Kollektivs insgesamt frühere, häufig asymptomatische Stadien ohne fortgeschrittenen Leberparenchymumbau vor (MELD-Score: 6,80 \pm 1,39). 


\subsubsection{Transaminasen}

Die NAFLD-Patienten dieses Kollektivs überschreiten den AST- und (insbesondere) den ALT-Referenzbereich, wie in zahlreichen Vorstudien bereits beobachtet, unabhängig vom Geschlecht z. T. deutlich (Haenle et al. 2006; Chen et al. 2007; Feng et al. 2017; Le et al. 2017; Zheng et al. 2017). Unterschiede zwischen NAFLD und AFLD bestehen bei alleiniger Betrachtung der Transaminasen lediglich für die ALT zwischen „non-lean-NAFLD“ und „AFLD“. Einschränkend muss diskutiert werden, dass bis zu $25 \%$ aller NAFLD-Fälle mit normwertigen Transaminasen einhergehen (Kälsch et al. 2015). Da im Falle der Studienpatienten häufig Transaminasenerhöhungen den Konsultationsgrund darstellten, ist bei alleiniger Fokussierung auf diese Laborparameter durch die Primärbehandler, eine Unterepräsentierung dieser Patientengruppe im Studienkollektiv zu erwarten. Diese Erkenntnis wird durch die Beobachtung gestützt, dass lediglich ein geringer Teil aller NAFLD-Patienten unter ärztlicher Kontrolle/Therapie steht, während die Dunkelziffer durch „Unterdiagnose“ weitaus höher liegt (Alexander et al. 2018; Blais et al. 2015; Sanyal 2018).

In der NAFLD-Subgruppenanalyse konnten keine Assoziationen für BMI und AST bzw. ALT, bzw. generelle Subgruppen-Unterschiede ermittelt werden (Kim et al. 2004; Feng et al. 2017; Alam et al. 2014; Kälsch et al. 2015), während ein Großteil von Studien einen Zusammenhang zwischen BMI und AST- bzw. prominenten ALT-Anstieg feststellen konnte (Margariti et al. 2012; Feng et al. 2014; Younossi et al. 2012; Mouzaki et al. 2013; Zheng et al. 2017). Ob diese Beobachtung der Stichprobengrößen des Kollektivs geschuldet ist sollte durch Vergrößerung des Kollektivs in Folgestudien geprüft werden.

Wird das Verhältnis zwischen AST und ALT im Sinne des de-Ritis-Quotienten hinzugezogen, ist eine Unterscheidung zwischen äthyltoxischer Leberschädigung und NAFLD eindeutig möglich (Kälsch et al. 2015). Aufgrund niedriger Kosten und einfacher Berechnung sollte er routinemäßig zur Differentialdiagnostik miteinbezogen werden. Inwieweit dies Indikator des (histologisch nachvollziehbaren) hepatischen Schädigungsmusters ist, bzw. prognostische Bedeutung erlangt ist aktuell unklar.

\subsubsection{Cholestaseparameter}

Der Bilirubinspiegel wird häufig genutzt, um das Ausmaß eines Leberschadens bei hepatologisch erkrankten Patienten zu bestimmen. Wesentliche Unterschiede bestehen nur zwischen AFLD und non-lean-NAFLD. Eine Differenzierung der NAFLD-Subgruppen untereinander ist, entgegen entsprechender Studien, nicht möglich (Hagström et al. 2018). Inwieweit dies durch die Stichprobengröße oder Selektion bedingt ist, ist anhand der Daten nicht zu klären.

Die gGT ist in sämtlichen Gruppen z. T. deutlich erhöht Eine Differenzierung ist in diesem Kollektiv zwischen „AFLD“ und „NAFLD“, jedoch nicht zwischen den NAFLDSubgruppen möglich (Alam et al. 2014; Bernhardt et al. 2018; Hagström et al. 2018). Die 
gGT wird mitunter als „Frühmarker“ einer NAFLD vorgeschlagen (Xu et al. 2013), zeigt sich jedoch häufig v. a. auch bei (fortgesetztem) Alkoholabusus z. T. führend erhöht und ist zur Differentialdiagnose nicht geeignet.

Die alkalische Phosphatase war bei NAFLD niedriger als bei AFLD. In der aktuellen Forschung wird der Relevanz der AP derzeit nur geringe Aufmerksamkeit gewidmet. Relevante Unterschiede werden nicht beschrieben (Feng et al. 2017).

\subsubsection{Lebersyntheseparameter}

Die Höhe des Albumins ist in sämtlichen Gruppen normwertig und unterscheidet sich gemäß Vergleichsstudien nicht zwischen den NAFLD-Subgruppen (Hagström et al. 2018), kann jedoch bei ausgeschlossener Mangelernährung als sehr spezifischer Marker der Lebersyntheseleistung im Sinne der Differentialdiagnose NAFLD und AFLD genutzt werden. Im Kollektiv lagen die Albuminspiegel signifikant niedriger. Ein Abweichen des NAFLD-Kollektivs zu Normkollektiven ist hingegen i. d. R. nicht zu erwarten (Le et al. 2017). Ein Erklärungsansatz ist der Zeitpunkt der Kontaktaufnahme, der bei AFLD durch die zunehmende Progression oder Dekompensation begründet ist. Indessen stellten sich die NAFLD-Patienten i. d. R. in einem asymptomatisch bzw. früheren, z. T. rein bildgebendoder laborchemisch-apparenten Stadium vor (s. o.). Ein Langzeit-Follow-up der betreffenden NAFLD-Patienten ist dennoch unbedingt zu empfehlen.

Die Analyse der erhobenen Gerinnungsparameter zeigt, bei geringen Abweichungen vom Normbereich. Unterschiede zwischen AFLD und NAFLD sind a. e. durch die unterschiedlichen Stadien der hepatischen Grunderkrankung erklärbar (Hahn 2013). Im NAFLD-Subgruppenvergleich zeigt sich, äquivalent einer kürzlich erschienenen Studie, keine Differenz (Hagström et al. 2018). Einschränkend ist zu erwähnen, dass die Einnahme antikoagulatorischer Medikamente in diesem Kontext ausgeschlossen werden muss. Im klinischen Alltag erschwert dies die Bewertung der Lebersyntheseeinschränkung bei alleiniger Betrachtung der Gerinnungssituation. Diesbezüglich sollte in jedem Fall vor Interpretation eine Mitbeurteilung des Serumalbuminspiegels bzw. z. B. der Cholinesterase erfolgen.

\subsubsection{Fett-/Lipidstatus}

Die NAFLD-Subgruppen zeigten in dieser Studie bezüglich des Fett-/Lipidstatus keine signifikanten Unterschiede untereinander. Bei Anstieg des BMI zeigt sich eine Steigerung des LDL-/Triglyceridspiegels bei gleichzeitigem Abfall des HDL, der sich jedoch nicht als signifikant erwies. Obwohl die aktuelle Forschung die NAFLD als hepatische Manifestation eines metabolischen Syndroms mit entsprechenden Veränderungen des Cholesterin- und Triglyceridstoffwechsels auffasst (Haenle et al. 2006; Treeprasertsuk et al. 2013; Roeb et al. 2015; Nishioji et al. 2015), fallen Studienergebnisse diesbezüglich kontrovers aus (Alam et al. 2014; Feng et al. 2014; Feng et al. 2017; Gonzalez-Cantero et al. 2018; Hagström et al. 2018; 
Kim et al. 2004). Insbesondere unter den lean-NAFLD-Patienten zeigten sich, entgegen anderer Studienergebnissen, keine dieser Auffälligkeiten (Bernhardt et al. 2018; Feng et al. 2014; Haenle et al. 2006; Zheng et al. 2017).

\subsubsection{Suchtmittel}

Die täglichen Alkoholkonsummengen waren in der AFLD-Gruppe niedrig und insgesamt nicht höher als in den NAFLD-Gruppen. Eine eindeutige Differenzialdiagnose zwischen NAFLD und AFLD wäre bei den festgestellten Durchschnittskonsummengen, gemäß Leitlinien-Cut-Off von 10 g/d (Frauen) bzw. 20 g/d (Männer), nicht gewährleistet (Roeb et al. 2015). Ausschlaggebend für diese Beobachtung ist Vorselektionierung, d.h. die hauptsächlich abstinente Lebensweise der AFLD-Patienten dieses Kollektivs, während AFLD-Patienten mit fortgesetztem Abusus im LZG nur selten vorstellig werden.

Eine Abstinenz wurde durch regelmäßige Kontrollen von Alkoholabbauprodukten (Ethylglucuronid, Ethylsulfat, CDT) verifiziert. Einschränkend muss diskutiert werden, dass auch in den anderen Gruppen der Alkoholkonsum überraschend niedrig, d. h. deutlich unter dem geschätzten Durchschnittskonsum der deutschen Allgemeinbevölkerung lag (World Health Organization 2012, 2014). Da keine Objektivierung des anamnestischen Alkoholkonsums möglich ist, kann eine Verzerrung der Ergebnisse durch unwahre Antworten, z. B. motiviert durch „soziale Erwünschtheit“, nicht ausgeschlossen werden. Andererseits wäre vorstellbar, dass hepatologisch vorselektionierte Kollektive sich durch eine vermehrte Sensibilisierung für die Vorteile eines maßvollen Alkoholkonsums/-karenz auszeichnen und häufiger dementsprechende Lifestylemodifikation umsetzen. Die in Studien z. T. diskutierte Hypothese ggf. positiver Effekte geringen Alkoholkonsums im Gegensatz zu völlig abstinenter Lebensweise bei NAFLD (Hagström et al. 2017; Jayasekara et al. 2014; Dunn et al. 2012) lassen sich aufgrund dieser Limitationen nicht überprüfen. Da aber gerade im Rahmen einer NASH bekannt ist, dass toxische Substanzen bereits bei niedrigen Dosierungen relevant sein können, ist nach aktuellem Wissensstand davon abzuraten (Ascha et al. 2010; Ekstedt et al. 2009; Chalasani et al. 2017).

Der Anteil aktiver bzw. ehemaliger Raucher liegt im Kollektiv ebenfalls deutlich unter den Werten der deutschen Allgemeinbevölkerung (Raucheranteil: ca. 24,5\% der Allgemeinbevölkerung, bzw. $27 \%$ der Männer und 20,8\% der Frauen) (Statistisches Bundesamt 2014; Zeiher et al. 2017; Haenle et al. 2006). AFLD-Patienten gaben lediglich häufiger als „,non-lean-NAFLD“-Patienten einen ehemaligen oder aktiven Nikotinabusus an. Im Vergleich zur „lean-NAFLD“ werden größere Stichproben zur Beurteilung benötigt. Eine Assoziation von Rauchen und AFLD (Schumann et al. 2001) bzw. ein erhöhtes NAFLD-Risiko bei Nikotinabusus wurde bereits beschrieben (Okamoto et al. 2018). Die Patientenangaben sind auch hier kritisch zu hinterfragen. 


\subsubsection{Lifestylefaktoren}

Ein Aspekt dieser Arbeit stellt die Fragestellung dar, inwieweit eine NAFLD, unter anderem im Vergleich zur AFLD bzw. die NAFLD-Subgruppen im direkten Vergleich, durch spezielle Lifestyle-assoziierte Besonderheiten gekennzeichnet sind. Einschränkend muss für alle betrachteten Parameter auf die fehlende Objektivierbarkeit hingewiesen werden. Die Patienten wurden im Rahmen des EK einer intensiven Lifestyle-Anamnese unterzogen, eine objektive Sicherung der Patientengaben war hingegen nicht möglich. Insoweit muss unterstellt werden, dass die Patientenangaben z. T. durch Faktoren wie „soziale Erwünschtheit" oder weitere Störfaktoren (s. o. ) ggf. nur bedingt auswertbar sind.

Signifikante Schwankungen bezüglich der täglichen Obst-/Fruchtsaftaufnahme bestehen im Kollektiv nicht, während aktuell die enge Assoziation zwischen fruktosereicher Ernährung und NAFLD diskutiert wird (Nobili et al. 2017; Roeb et al. 2015). Insbesondere im Vergleich zur AFLD ist, bezugnehmend auf diese Studienergebnisse, im Studienkollektiv nicht von grundsätzlich verschiedenen Lifestylegewohnheiten auszugehen.

Die Verzehrmengen von Softdrinks unterscheiden sich im Kollektiv ebenfalls nicht, sodass die in zahlreichen Studien beobachtete Assoziation zwischen Softdrinkkonsum und NAFLD in der direkten Differentialdiagnose zur AFLD nicht bestätigt werden kann (Zelber-Sagi et al. 2007; Assy et al. 2008; Abid et al. 2009; Malik et al. 2010; Hashemi Kani et al. 2014).

Die AFLD-Patienten erwiesen sich, übereinstimmend mit Vorstudien, tendenziell als die sportlich inaktivste Gruppe (Schumann et al. 2001. Indessen waren non-lean-NAFLDPatienten in dieser Untersuchung sportlich am aktivsten. Ihr Bewegungsaufwand liegt insgesamt auf dem Niveau der deutschen Allgemeinbevölkerung (Haenle et al. 2006), während ein Bewegungsmangel gemeinhin als ätiologisch relevant für die NAFLD angenommen wird (Oddy et al. 2013; Bae et al. 2012; Kistler et al. 2011; Zelber-Sagi et al. 2008).

Es wird zunehmend eine potenziell protektive Bedeutung regelmäßigen Kaffeekonsums diskutiert (Birerdinc et al. 2012; Yesil und Yilmaz 2013; Marventano et al. 2016; Gupta et al. 2015). Im untersuchten Kollektiv stellte der Kaffeekonsum kein Differenzierungsparameter dar. Zur Evaluierung v. a. auch der progressionshemmenden Effekte wäre eine große prospektive Fall-Kontroll-Studie über voraussichtlich mehrere Jahrzehnte notwendig. 


\subsection{Kurzzeit-Follow-up der lean-NAFLD im Vergleich zur non-lean- NAFLD}

Die ermittelten NAFLD-Patienten wurden im Mittel nach 165,1 \pm 78,72 Tagen in der Ambulanz reevaluiert. Alle Patienten wurden bei Erstkontakt standardisiert über sinnvolle Lebensstilmodifikationen aufgeklärt. Insgesamt stellen sich $\Delta$ BMI, $\triangle$ AST, $\triangle$ ALT und $\Delta$ gGT nicht unterschiedlich zwischen den Gruppen „lean“- bzw. „non-lean“-NAFLD dar. Inwieweit die Beobachtung bezüglich der $\Delta \mathrm{AP}$ relevant einzuschätzen ist, ist aufgrund der bisherigen Datenlage nicht einzuschätzen.

Insbesondere das $\Delta$ BMI unterscheidet sich nicht zwischen den gewählten Gruppen. Ein signifikanter Gewichtsverlust konnte, wie bereits in einer kürzlich publizierten Studie mit 11-Jahres-Follow-up beschrieben, für das Gesamtkollektiv in keiner Subgruppe festgestellt werden (Bürckner 2017).

Interessanterweise waren die o.g. Laborparameter, hier v. a. die Transaminasen insbesondere bei non-lean-NAFLD, regredient, während sich bei lean-NAFLD (AST/ALT konstant) keine Veränderungen zeigten. Der im Mittel fehlende Gewichtsverlust mag in der Kürze des follow-ups ggf. nicht messbar gewesen sein. Der laborchemische Effekt kann sich aber durch andere Lebensstilanpassungen (reduzierter Zucker- und Fettkonsum, gesteigerte Aktivität, etc.) erklären. Der fehlende Effekt bei lean-NAFLD-Patienten kann auch durch nur sehr kleine absolute Effekte der insgesamt vergleichsweise gering auffälligen Laborparameter erklärbar sein.

Die standardisierte Beratung im Rahmen der Leberambulanz kann folglich kurzfristig zu einer laborchemischen Verbesserung v. a. der non-lean-NAFLD führen. Derartige Untersuchungen gibt es zum Zeitpunkt der vorliegenden Arbeit nicht. Möglicherweise ist die mutmaßliche hohe Motivationslage bei Patienten die eine spezialisierte Hochschulambulanz aufsuchen und/oder das Bewusstmachen der Einflussfaktoren ein wichtiger Faktor für die beobachteten Effekte. Längerfristige Beobachtungen wären interessant, um einerseits zu prüfen wie lange diese Effekte anhalten bzw. ob sich die Effekte noch verstärken oder andererseits für das Gesamtkollektiv auch eine Senkung des mittleren BMI erzielt werden kann. Der positive Effekt der Lebensstilmodifikationen, wie sie auch in der deutschen Leitlinie erläutert und empfohlen werden, bestätigt sich auch in dieser Untersuchung. Es ist zu erwarten, dass die Effekte durch eine Kurzberatung im Rahmen einer Leberambulanz durch eine professionelle und begleitende Ernährungsberatung intensiviert werden können. Entsprechende Vergleichsstudien wären zur Klärung dieser Frage notwendig. 


\subsection{Effekte einer standardisierten Kurzberatung im Kurzzeitintervall}

Einen wichtigen Aspekt dieser Arbeit stellt die Bewertung des Effekts von Lifestyleinterventionen im Kurzzeit-Follow-up (165,1 \pm 78,72 Tage) als aktuell wichtigste Therapieoption dar (Byrne und Targher 2014; Roeb et al. 2015; Weiss et al. 2014).

Im Studienkollektiv kam es in der „Gewichtsverlust“-Gruppe zu einer BMI-Reduktion von 1,67 BMI-Punkten bzw. 5,50 \% des Körpergewichts und messbaren Regredienz der ALT bzw. gGT. Insgesamt wird die Gewichtsreduktion als wichtigste (Lifestyle-)Intervention angesehen, z. T. mit Erreichen vollständiger Remissionen (Thoma et al. 2012; Weiss et al. 2014). Positive Effekte treten v. a. bei einem Körpergewichtsverlust von ca. 3 - $10 \%$ auf (Promrat et al. 2010; Chalasani et al. 2017; Thoma et al. 2012; Wong et al. 2018; Ratziu et al. 2010), welcher im untersuchten Kollektiv z. T. erzielt werden konnte. Im Detail konnte gezeigt werden, dass sich bei Gewichtsverlust ein signifikanter Rückgang der ALT und gGT einstellte. Diese Daten decken sich mit Erkenntnissen anderer Autoren (Ratziu et al. 2010; Hohenester et al. 2018; Shidfar et al. 2018; Wong et al. 2018). Diskussionswürdig ist, dass lediglich laborchemische Methoden zur Verlaufsbeurteilung genutzt wurden und Aussagen über die feinhistologische Beschaffenheit des Leberparenchyms nicht vorliegen. Dem zu entgegnen sind der hohe Aufwand und die Risiken einer Biopsie (Blutungskomplikationen, Schmerzen, Infektionen, etc.) und die häufig fehlende therapeutische Konsequenz, die zunehmend zur Verdrängung der Biopsie in diesem Kontext beiträgt (s. o.).

Gemäß neusten Studien konnte der positive Effekt eines Gewichtsverlustes auch für normalgewichtige Kollektive nachvollzogen werden (Wong et al. 2018). Inwieweit diese Beobachtung für die Allgemeinheit der normalgewichtigen NAFLD-Patienten gilt bzw. diese Erkenntnis in Folgestudien replizierbar ist, ist aktuell unklar. Festzustellen ist, dass insbesondere die Entwicklung eines Untergewichts aufgrund inhärenter Risiken (vorzeitige Osteoporose, negative Auswirkungen auf das hormonelle System, erhöhte Sterblichkeit im Vergleich zur normalgewichtigen Bevölkerung) zu vermeiden ist und die positiven Effekte einer NAFLD-Regression nicht aufwiegen. Nicht zuletzt dürfte die Empfehlung zur Gewichtsabnahme in lean-Kollektiven deutlich schlechter zu vermitteln sein. Letztendlich bleibt zudem unklar, inwieweit die ätiologischen Erklärungsansätze der non-lean-NAFLD (Assoziation zum metabolischen Syndrom, erhöhter Softdrink-/Fruchtsaftkonsum, Bewegungsmangel) (Roeb et al. 2015; Caldwell et al. 1999; Rajindrajith et al. 2017; Denzer et al. 2009, Assy et al. 2008; Abid et al. 2009; Malik et al. 2010; Hashemi Kani et al. 2014) auf die lean-NAFLD in diesem Ausmaß übertragbar sind oder andere zum gegenwärtigen Zeitpunkt noch unbekannte Faktoren als maßgeblich zu bewerten sind.

Bei Patienten ohne Gewichtsreduktion bzw. Gewichtszunahme schreitet die Progression der NAFLD und der assoziierten Erkrankungen (Diabetes mellitus Typ 2, art. Hypertonie, etc.) fort. Als Minimalkonsens gilt, dass eine Gewichtszunahme zu vermeiden ist. Diesbezüglich sollten weitere Studien sich der Frage annehmen, wie hoch die negativen Effekte einer Gewichtszunahme im Vergleich zur Gewichtsstabilisierung prognostisch sind. 


\section{$5 \quad$ Zusammenfassung}

Die nichtalkoholische Fettlebererkrankung (NAFLD) hat in den letzten Jahren eine deutliche Zunahme der Prävalenz und Inzidenz verzeichnen können. Diese Arbeit konnte zeigen, dass aktuell die NAFLD den häufigsten Grund für eine Erstvorstellung in der hepatologischen Hochschulambulanz der Universitätsmedizin Göttingen darstellt. Mehr als ein Drittel aller ausgewerteten Studienpatienten erhielt die Diagnose NAFLD, während die Zahl der viralen Hepatitiden und der äthyltoxischen Hepatopathien im Vergleich kontinuierlich abnimmt. Die NAFLD ist vor dem Hintergrund ihrer hohen Prävalenz in der Allgemeinbevölkerung zunehmend relevant und insbesondere aufgrund ihrer potenziellen Progression in gegebenenfalls maligne Verlaufsformen sowohl für den Betroffenen als auch gesundheitspolitisch und ökonomisch nicht zu unterschätzen.

Zunehmend wird in den letzten Jahren die Sonderform der lean-NAFLD, d. h. NAFLD bei normal- oder untergewichtigen Patienten, betrachtet. Diese Arbeit konnte zeigen, dass ein signifikanter Anteil an NAFLD-Patienten normalgewichtig ist und erhöhtes Körpergewicht bzw. metabolische Risikofaktoren allein nicht in der Lage sind die Gesamtheit der NAFLDPatienten abzubilden.

Im klinischen Alltag ist die exakte Differentialdiagnose zwischen alkoholischer Fettlebererkrankung (AFLD) und NAFLD eine grundlegende Herausforderung. Diese Studie konnte zeigen, dass anthropometrische Daten (Alter, body mass index, Blutdruck, Geschlecht, etc.), die in anderen Veröffentlichungen dazu dienen konnten NAFLDPatienten von einem gesunden Vergleichskollektiv zu unterscheiden, im Vergleich zwischen AFLD und NAFLD häufig versagen. Insbesondere lean-NAFLD-Patienten weisen bereits zu Normalkollektiven nur geringe Unterschiede auf und diese Parameter sind daher ungeeignet, um diese Sondergruppe zu erfassen. Diese Beobachtung lässt sich ebenfalls für viele etablierte laborchemische Parameter treffen. Einsetzbar sind vor allem Parameter wie die Alanin-Aminotransferase, der de-Ritis-Quotient, Gesamtalbumin oder der Quickwert, da sie die Differentialdiagnostik unterstützen und in allen medizinischen Bereichen verfügbar bzw. einfach zu erheben sind.

Eine der Kernfragen dieser Arbeit war, inwieweit spezielle Lifestylefaktoren in einem hepatologischen Kollektiv bzw. innerhalb des NAFLD-Kollektivs unterschiedlich präsent

waren. Zahlreiche Studien deuten u. a. eine verminderte sportliche Aktivität, hohe Fruktoseaufnahme, etc. in NAFLD-Kollektiven im Vergleich $\mathrm{zu}$ gesunden Vergleichsgruppen an. Im untersuchten Kollektiv ist primär kein Unterschied feststellbar. Die Lifestyleanamnese kann somit oft nicht zuverlässig dazu dienen, die Differenzierung zwischen AFLD und NAFLD zu unterstützen. Unterschiede sind auch innerhalb des NAFLD-Kollektivs nicht nachzuweisen. Trotz dieser Einschränkung ist die Lifestyleanamnese eine wichtige Kernfrage in der therapeutischen Entscheidungsfindung. 
Aktuell ist für die NAFLD eine medikamentöse Therapie nicht verfügbar. Die aktuellen nationalen bzw. internationalen Leitlinien empfehlen eine Lifestyleintervention bestehend aus Gewichtsverlust, Ernährungsumstellung (zucker- und fettarm) und vermehrter körperlicher Aktivität. Daten über die positiven Effekte einer solchen Intervention liegen aktuell in wachsendem Ausmaß vor.

Die Patienten dieser Studie wurden im Rahmen der Ambulanzkonsultation über die o. g. Interventionsmöglichkeiten informiert und bezüglich ihrer Umsetzung geschult. Diese Arbeit konnte zeigen, dass bereits eine Intervention im Kurzintervall signifikante Effekte erzielen kann. Bereits die einmalige, standardisierte Kurzberatung in einer hepatologischen Hochschulambulanz ist geeignet, um einen großen Anteil der Patienten zu einer Lifestyleintervention $\mathrm{zu}$ motivieren. Wird eine Lifestyleintervention erfolgreich durchgeführt, kommt es zu signifikanten Rückgängen der zuvor erhöhten Transaminasen bzw. der Gamma-Glutamyltranspeptidase. Bereits ein geringerfügigerer Gewichtsverlust von durchschnittlich 5,5 \% des Körpergewichts genügte, um ein laborchemisches Improvement zu erzielen. Langzeituntersuchungen sollten sich der Frage annehmen, inwieweit dieser Effekt dauerhaft ist und prognostische Bedeutung erlangt. Insbesondere der Langzeitvergleich mit Kollektiven ohne Lifestyleanpassung sollte angestrebt werden, um die (Langzeit-)Effekte einzuschätzen.

Einschränkend muss an dieser Stelle auf Limitationen dieser Studie hingewiesen werden. Die Größe des Patientenkollektivs sowie die Vorselektionierung lässt lediglich Aussagen über das Kollektiv einer hepatologischen Hochschulambulanz zu. Inwieweit die beobachteten Ergebnisse auf die Patienten im nichtvorselektionierten Umfeld, z. B. einer hausärztlichen Praxis bzw. auf NAFLD-Patienten im Generellen zu übertragen sind, ist aktuell unklar. 


\section{Anhang}

\section{Patientenfragebogen}

Name, Vorname:

Geb.-Datum:

Adresse:

Tel.-Nr.:
UNIVERSITÄTSMEDIZIN $\equiv \mathbf{U}$
GÖTTINGEN $=$

$\underset{\text { Göttingen }}{\text { LeberZentrum }} \mathbf{Z G}$

Patientenfragebogen

Neuvorstellungen

Datum (Ausfüllzeitpunkt):

Mit welchen Beschwerden / welchem Verdacht stellen Sie sich bei uns vor?

(Bitte hier kurz erläutern)

Allgemeine Daten

\begin{tabular}{|l|l|}
\hline Alter & \\
\hline Körpergröße (in cm) & \\
\hline Körpergewicht (in kg) & \\
\hline Aktueller Beruf & \\
\hline \begin{tabular}{l} 
Ggf. früherer Beruf \\
\hline $\begin{array}{l}\text { Höchster Bildungsabschluss (z.B. } \\
\text { Hauptschule, Abitur, Studium, Promotion) }\end{array}$
\end{tabular} \\
\hline
\end{tabular}

\section{Vorerkrankungen und operative Eingriffe}

Bitte listen Sie hier alle bekannten Vorerkrankungen und operativen Eingriffe mit dem Jahr der Erstdiagnose, bzw. OP auf! Bitte bringen Sie ggf. auch aktuelle Arztbriefe mit! 


\section{Aktuelle Symptome}

\begin{tabular}{|l|l|l|l|}
\hline $\begin{array}{l}\text { Bitte kreuzen Sie Zutreffendes an und erläutern } \\
\text { Sie ggf. genauer. }\end{array}$ & Ergänzende Information & Nain \\
\hline Müdigkeit / Abgeschlagenheit & & & \\
\hline Leichte Erschöpfbarkeit & & & \\
\hline Konzentrations- und Gedächtnisstörung & & & \\
\hline Gelenkschmerzen (ggf. welche Gelenke?) & & & \\
\hline Juckreiz (ggf. wo?) & & & \\
\hline Mundtrockenheit & & & \\
\hline Gelbfärbung (ggf. wann?) & & & \\
\hline Bauchwasser (Aszites) & & & \\
\hline Gewichtszunahme (ggf. wieviel und seit wann?) & & & \\
\hline Gewichtsabnahme (ggf. wieviel und seit wann?) & & & \\
\hline Vermehrtes Schwitzen & & & \\
\hline Regelmäßiges Fieber & & & \\
\hline Wie viele Stuhlgänge haben Sie pro Tag? & & & \\
\hline Obstipation / Verstopfung & & & \\
\hline Schlafstörungen & & & \\
\hline Häufig „blaue Flecken“ & & & \\
\hline Langes Bluten nach Verletzungen & & & \\
\hline Blutübertragungen / Transfusionen erhalten & & & \\
\hline Tätowierungen und/oder Piercings (Lokalisation) & & & \\
\hline
\end{tabular}




\section{Medikamente}

Bitte listen Sie Ihre aktuellen Medikamente mit genauer Dosierung an! Sie können auch alternativ einen aktuellen hausärztlichen Medikamentenplan anhängen.

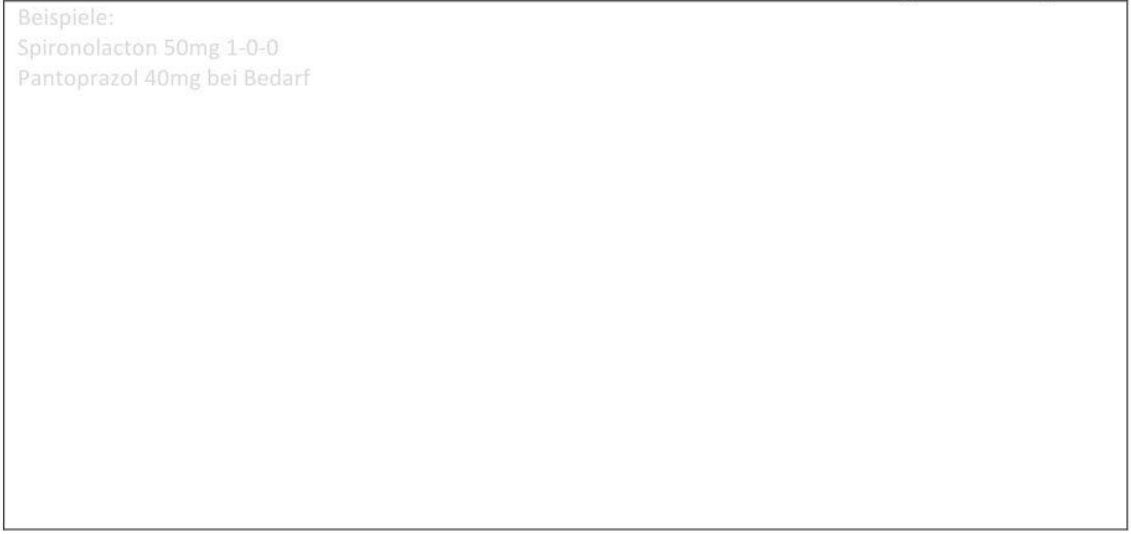

\section{Allergien}

Leiden Sie unter Allergien? Bitte listen Sie hier alle Allergien und Unverträglichkeiten auf und beschreiben Sie die Art der Reaktion!

\section{Leberspezifische Vortherapien}

Hatten Sie bereits Therapien, welche die Leber betreffen? Zum Beispiel antivirale Therapien einer Hepatitis B oder Hepatitis C? Behandlung einer Autoimmunhepatitis? Bitte listen Sie Ihre Vortherapien mit Medikamentennamen und genauem Zeitraum hier auf! 


\section{Genussmittel (bitte genau angeben)}

\begin{tabular}{|c|l|l|l|}
\hline $\begin{array}{l}\text { Bitte kreuzen Sie Zutreffendes an bzw. } \\
\text { erläutern sie genauer. }\end{array}$ & Ergänzende Information & Ja \\
\hline Alkohol & & & \\
\hline - Was trinken Sie? & & & \\
\hline - An wieviel Tagen pro Woche? & & & \\
\hline - Wieviel pro Tag? (z.B. 4 Fl. Bier á 0,33L) & & & \\
\hline Rauchen & & & \\
\hline$-\quad$ Seit wann? & & & \\
\hline - Was rauchen Sie? (z.B. Zigaretten) & & & \\
\hline - Wie viel pro Tag? & & & \\
\hline Drogen & & & \\
\hline$-\quad$ Welche? & & & \\
\hline - Seit wann? / bis wann? & & & \\
\hline Andere Genussmittel & & & \\
\hline Kaffee (ggf. wie viele Tassen pro Tag?) & & & \\
\hline
\end{tabular}

\section{Lifestyle}

\begin{tabular}{|c|c|}
\hline $\begin{array}{l}\text { Wie ernähren Sie sich? } \\
\text { (vegan, vegetarisch, Fleischesser) }\end{array}$ & \\
\hline$-\quad \begin{array}{l}\text { An wieviel Tagen pro Woche essen Sie } \\
\text { ggf. Fleisch? }\end{array}$ & \\
\hline$-\quad \begin{array}{l}\text { An wieviel Tagen pro Woche essen Sie } \\
\text { ggf. Fisch? }\end{array}$ & \\
\hline Obstkonsum & \\
\hline$-\quad \begin{array}{l}\text { An wieviel Tagen pro Woche essen Sie } \\
\text { Obst? }\end{array}$ & \\
\hline $\begin{array}{l}\text { Wie viele Teile durchschnittlich? } \\
\text { (z.B. 2 Äpfel) }\end{array}$ & \\
\hline
\end{tabular}




\begin{tabular}{|c|c|}
\hline Wieviel Liter trinken Sie pro Tag? & \\
\hline$-\quad \begin{array}{l}\text { Wieviel Fruchtsaft/Smoothies trinken } \\
\text { Sie pro Tag? }\end{array}$ & \\
\hline$-\quad \begin{array}{l}\text { Wieviel Softdrinks (Cola, Limo, etc.) } \\
\text { trinken Sie pro Tag? }\end{array}$ & \\
\hline Sport $\quad$ & \\
\hline$-\quad \begin{array}{l}\text { Sind Sie beruflich viel in Bewegung? } \\
\text { (wenn ja: in welcher Form?) }\end{array}$ & \\
\hline$-\quad \begin{array}{l}\text { An wieviel tagen pro Woche treiben Sie } \\
\text { Sport? (mind. 30 Minuten) }\end{array}$ & \\
\hline$\quad$ Welchen Sport betreiben Sie? & \\
\hline
\end{tabular}

\section{Auslandsreisen}

Waren Sie im (außereuropäischen) Ausland? Bitte listen Sie ggf. auf, wann Sie in welchem Land waren.

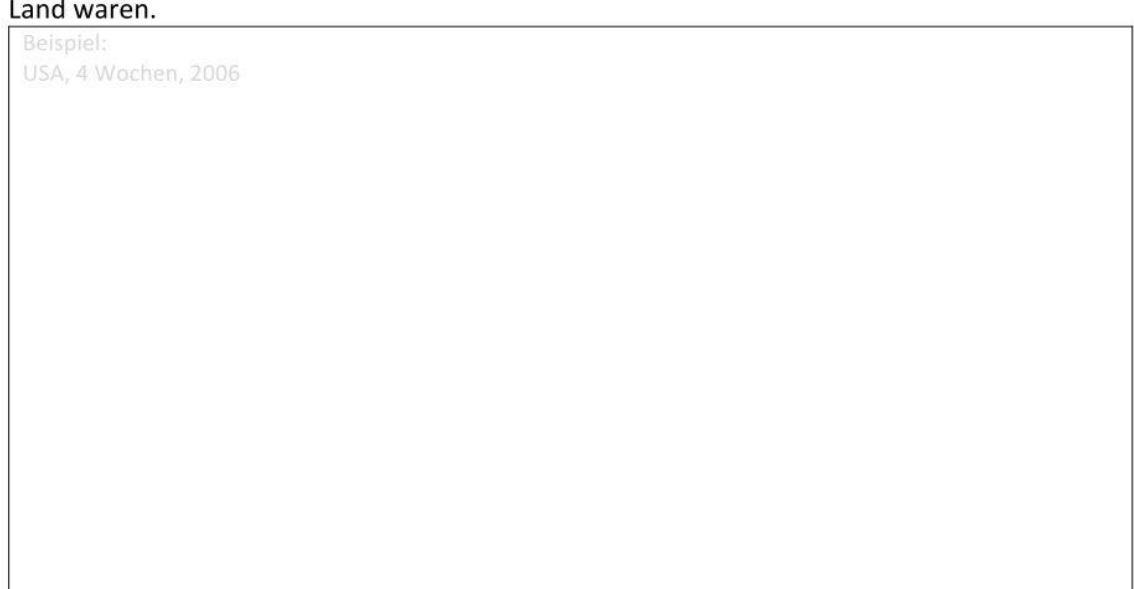

Impfungen

Bitte bringen Sie im Zweifel Ihren Impfausweis mit!

\begin{tabular}{|l|l|l|c|}
\hline & Wann zuletzt? & Nein & Ja \\
\hline Sind Sie gegen Hepatitis A geimpft? & & & \\
\hline Sind Sie gegen Hepatitis B geimpft? & & & \\
\hline $\begin{array}{l}\text { Sind Sie gegen die Virusgrippe (Influenza) } \\
\text { geimpft? }\end{array}$ & & & \\
\hline
\end{tabular}


Familienvorgeschichte

Hier geht es um Erkrankungen bei direkten Verwandten, nicht um Ihre eigenen. Bitte geben Sie möglichst genau an, welcher Verwandte ggf. betroffen war und welche Diagnose vorlag.

\begin{tabular}{|l|l|l|l|}
\hline $\begin{array}{l}\text { Erkrankung bei } \\
\text { Familienangehörigen }\end{array}$ & Wer? Wann? Folgen? & Nein & Ja \\
\hline Lebererkrankungen & & & \\
\hline $\begin{array}{l}\text { Diabetes mellitus } \\
\text { („Zuckerkrankheit“) }\end{array}$ & & & \\
\hline Bluthochdruck & & & \\
\hline $\begin{array}{l}\text { Herzerkrankungen (z.B. } \\
\text { Herzinfarkt) }\end{array}$ & & & \\
\hline Schlaganfall & & & \\
\hline $\begin{array}{l}\text { Krebserkrankungen / } \\
\text { Tumorerkrankungen }\end{array}$ & & & \\
\hline Fettleibigkeit & & & \\
\hline $\begin{array}{l}\text { Osteoporose / } \\
\text { „Knochenschwund“ }\end{array}$ & & & \\
\hline Rheumatische Erkrankungen & & & \\
\hline Genetische Erkrankungen & & & \\
\hline Sonstiges (bitte erläutern) & & & \\
\hline
\end{tabular}




\section{$7 \quad$ Literaturverzeichnis}

Abdeldyem SM, Goda T, Khodeir SA, Abou Saif S, Abd-Elsalam S (2017): Nonalcoholic fatty liver disease in patients with acute ischemic stroke is associated with more severe stroke and worse outcome. J Clin Lipidol 11, 915-919

Abid A, Taha O, Nseir W, Farah R, Grosovski M, Assy N (2009): Soft drink consumption is associated with fatty liver disease independent of metabolic syndrome. J Hepatol $\underline{51}, 918-924$

Agopian VG, Kaldas FM, Hong JC, Whittaker M, Holt C, Rana A, Zarrinpar A, Petrowsky H, Farmer D, Yersiz H et al. (2012): Liver transplantation for nonalcoholic steatohepatitis: the new epidemic. Ann Surg 256, 624-633

Akyuz U, Yesil A, Yilmaz Y (2015): Characterization of lean patients with nonalcoholic fatty liver disease: potential role of high hemoglobin levels. Scand J Gastroenterol $\underline{50}$, 341-346

Alam S, Gupta UD, Alam M, Kabir J, Chowdhury ZR, Alam AK (2014): Clinical, anthropometric, biochemical, and histological characteristics of nonobese nonalcoholic fatty liver disease patients of Bangladesh. Indian J Gastroenterol $\underline{33}$, 452-457

Alberti KG, Eckel RH, Grundy SM, Zimmet PZ, Cleeman JI, Donato KA, Fruchart JC, James WP, Loria CM, Smith SC Jr et al. (2009): Harmonizing the metabolic syndrome: a joint interim statement of the Inter-national Diabetes Federation Task Force on Epidemiology and Prevention; National Heart, Lung, and Blood Institute; American Heart Association; World Heart Federation; International Atheroscle-rosis Society; and International Association for the Study of Obesity. Circulation 120, 1640-1645

Alexander M, Loomis AK, Fairburn-Beech J, van der Lei J, Duarte-Salles T, Prieto-Alhambra D, Ansell D, Pasqua A, Lapi F, Rijnbeek P et al. (2018): Real-world data reveal a diagnostic gap in non-alcoholic fatty liver disease. BMC Med $\underline{13}, 130$

Anania C, Perla FM, Olivero F, Pacifico L, Chiesa C (2018): Mediterranean diet and nonalcoholic fatty liver disease. World J Gastroenterol. 24, 2083-2094

Anty R, Marjoux S, Iannelli A, Patouraux S, Schneck AS, Bonnafous S, Gire C, Amzolini A, BenAmor I, Saint-Paul MC et al. (2012): Regular coffee but not espresso drinking is protective against fibrosis in a cohort mainly composed of morbidly obese European women with NAFLD undergoing bariatric surgery. J Hepatol $\underline{57}$, 1090-1096

Anurag L, Aniket S, Shalik J, Amarja L, Dhananjay R, Sachin J (2015): Non-alcoholic fatty liver disease prevalence and associated risk factors - A study from rural sector of Maharashtra. Trop Gastroenterol 36, 25-30

Ascha MS, Hanouneh IA, Lopez R, Tamimi TAR, Feldstein AF, Zein NN (2010): The incidence and risk factors of hepatocellular carcinoma in patients with nonalcoholic steatohepatitis. Hepatology $\underline{51}, 1972-1978$

Asgari-Taee F, Zerafati-Shoae N, Dehghani M, Sadeghi M, Baradaran HR, Jazayeri S (2018): Association of sugar sweetened beverages consumption with non-alcoholic fatty liver disease. A systematic review and meta-analysis. Eur J Nutr $\underline{58}, 1759-1769$ 
Assimakopoulos K, Karaivazoglou K, Tsermpini EE, Diamantopoulou G, Triantos C (2018): Quality of life in patients with nonalcoholic fatty liver disease. A systematic review. J Psychosom Res 112, 73-80

Assy N, Nasser G, Kamayse I, Nseir W, Beniashvili Z, Djibre A, Grosovski M (2008): Soft Drink Consumption Linked with Fatty Liver in the Absence of Traditional Risk Factors. Can J Gastroenterol 22, 811-816

Bae JC, Rhee EJ, Lee WY, Park SE, Park CY, Oh KW, Park SW, Kim SW (2011): Combined effect of nonalcoholic fatty liver disease and impaired fasting glucose on the development of type 2 diabetes: a 4-year retrospective longitudinal study. Diabetes Care $\underline{34}$, 727-729

Bae JC, Suh S, Park SE, Rhee EJ, Park CY, Oh KW, Park SW, Kim SW, Hur KY, Kim JH et al. (2012): Regular exercise is associated with a reduction in the risk of NAFLD and decreased liver en-zymes in individuals with NAFLD independent of obesity in Korean adults. PLoS One 7 , e46819

Bae JC, Han JM, Cho JH, Kwon H, Park SE, Park CY, Lee WY, Oh KW, Kwon S, Park SW (2018). The persistence of fatty liver has a differential impact on the development of diabetes: The Kangbuk Samsung Health Study. Diabetes Res Clin Pract 135, 1-6

Bae M, Park YK, Lee JY (2017): Food components with antifibrotic activity and implications in prevention of liver disease. J Nutr Biochem $\underline{55}, 1-11$

Bambha K, Wilson LA, Unalp A, Loomba R, Neuschwander-Tetri BA, Brunt EM, Bass NM; Nonalcoholic Steatohepatitis Clinical Research Network (NASH CRN) (2014): Coffee consumption in NAFLD patients with lower insulin resistance is associated with lower risk of severe fibrosis. Liver Int $\underline{34}, 1250-1258$

Baumeister SE, Völzke H, Marschall P, John U, Schmidt CO, Flessa S, Alte D (2008): Impact of fatty liver disease on health care utilization and costs in a general population: a 5 -year observation. Gastroenterology 134, 85-94

Bernhardt P, Kratzer W, Schmidberger J, Graeter T, Gruener B; EMIL Study Group (2018): Laboratory parameters in lean NAFLD. Comparison of subjects with lean NAFLD with obese subjects without hepatic steatosis. BMC Res Notes $\underline{11}, 101$

Birerdinc A1, Stepanova M, Pawloski L, Younossi ZM (2012): Caffeine is protective in patients with non-alcoholic fatty liver disease. Aliment Pharmacol Ther 35, 76-82

Blachier M, Leleu H, Peck-Radosavljevic M, Valla DC, Roudot-Thoraval F (2013): The burden of liver disease in Europe: a review of available epidemiological data. J Hepatol $\underline{58}, 593-608$

Blais P, Husain N, Kramer JR, Kowalkowski M, El-Serag H, Kanwal F (2015): Nonalcoholic fatty liver disease is underrecognized in the primary care setting. Am J Gastroenterol 110, 10-4

Bohte AE, Bipat S, Stoker J, van Werven JR (2011): The diagnostic accuracy of US, CT, MRI and 1H-MRS for the evaluation of hepatic steatosis compared with liver biopsy. Eur Radiol 21, 87 97

Bürckner C: Follow-up über 11 Jahre von Probanden mit "Lean-NAFLD". Med. Diss. Ulm 2017 
Buzzetti E, Lombardi R, Luca L, Tsochatzis EA (2015): Noninvasive Assessment of Fibrosis in Patients with Nonalcoholic Fatty Liver Disease. World J Gastroenterol 21, 11044-11052

Byrne CD, Targher G (2014): Ectopic fat, insulin resistance, and nonalcoholic fatty liver disease: implications for cardiovascular disease. Arterioscler Thromb Vasc Biol 쓰, 1155-1161

Caldwell SH, Oelsner DH, Iezzoni JC, Hespenheide EE, Battle EH, Driscoll CJ (1999): Cryptogenic cirrhosis: clinical characterization and risk factors for underlying disease. Hepatology 29 , 664-669

Cauchy F, Zalinski S, Dokmak S, Fuks D, Farges O, Castera L, Paradis V, Belghiti J (2013): Surgical treatment of hepatocellular carcinoma associated with the metabolic syndrome. Br J Surg 100, $113-121$

Chalasani N, Younossi Z, Lavine JE, Charlton M, Cusi K, Rinella M, Harrison SA, Brunt EM, Sanyal AJ (2017): The Diagnosis and Management of Nonalcoholic Fatty Liver Disease: Practice Guidance from the American Association for the Study of Liver Diseases. Hepatology $\underline{67}, 328-357$

Chen CH, Huang MH, Yang JC, Nien CK, Yang CC, Yeh YH, Yueh SK (2007): Prevalence and etiology of elevated serum alanine aminotransferase level in an adult population in Taiwan. J Gastroenterol Hepatol 22, 1482-1489

Chen JY, Hsu CT, Liu JH, Tung TH (2014): Clinical predictors of incident gallstone disease in a Chinese population in Taipei, Taiwan. BMC Gastroenterol 14, 83

Choi JH, Rhee EJ, Bae JC, Park SE, Park CY, Cho YK, Oh KW, Park SW, Lee WY (2013): Increased risk of type 2 diabetes in subjects with both elevated liver enzymes and ultrasonographically diagnosed nonalcoholic fatty liver disease: a 4-year longitudinal study. Arch Med Res $\underline{44}, 115-120$

Cohen JC, Horton JD, Hobbs HH (2011): Human fatty liver disease: old questions and new insights. Science $\underline{332}, 1519-1523$

Corey KE, Klebanoff MJ, Tramontano AC, Chung RT, Hur C (2016): Screening for Nonalcoholic Steatohepatitis in Individuals with Type 2 Diabetes: A Cost-Effectiveness Analysis. Dig Dis Sci $\underline{61}, 2108-2117$

Crossan C, Tsochatzis EA, Longworth L, Gurusamy K, Davidson B, Rodríguez-Perálvarez M, Mantzoukis K, O'Brien J, Thalassinos E et al. (2015): Cost-effectiveness of non-invasive methods for assessment and monitoring of liver fibrosis and cirrhosis in patients with chronic liver disease: systematic review and economic evaluation. Health Technol Assess 19, 1-409

Crow D (2014): Big pharma bets billions on 'silent' liver disease. Financial Times. https://www.ft.com/content/e6ab5da6-f3c9-11e6-8758-6876151821a6; abgerufen am 20.09.2019

Cusi K (2012): Role of obesity and lipotoxicity in the development of nonalcoholic steatohepatitis: pathophysiology and clinical implications. Gastroenterology 142, 711-725 
D'Avola D, Labgaa I, Villanueva A, (2016): Natural history of nonalcoholic steatohepatitis/nonalcoholic fatty liver disease-hepatocellular carcinoma. Magnitude of the problem from a hepatology clinic perspective. Clin Liver Dis $\underline{8}, 100-104$

Denzer C, Thiere D, Muche R, Koenig W, Mayer H, Kratzer W, Wabitsch M (2009): Genderspecific prevalences of fatty liver in obese children and adolescents: roles of body fat distribution, sex steroids, and insulin resistance. J Clin Endocrinol Metab 94, 3872-3881

Dunn W, Sanyal AJ, Brunt EM, Unalp-Arida A, Donohue M, McCullough AJ, Schwimmer JB (2012): Modest alcohol consumption is associated with decreased prevalence of steatohepatitis in patients with non-alcoholic fatty liver disease (NAFLD). J Hepatol $\underline{57}$, 384-391

Ekstedt M, Franzén LE, Mathiesen UL, Thorelius L, Holmqvist M, Bodemar G, Kechagias S (2006): Long-term follow-up of patients with NAFLD and elevated liver enzymes. Hepatology 44, 865-73

El-Serag HB, Tran T, Everhart JE (2004): Diabetes increases the risk of chro-nic liver disease and hepatocellular carcinoma. Gastroenterology $126,460-468$

Eng K, Lopez R, Liccardo D, Nobili V, Alkhouri N (2014): A non-invasive prediction model for non-alcoholic steatohepatitis in paediatric patients with non-alcoholic fatty liver disease. Dig Liver Dis $\underline{46}, 1008-1013$

Ertle J, Dechêne A, Sowa JP, Penndorf V, Herzer K, Kaiser G, Schlaak JF, Gerken G, Syn WK, Canbay A (2011): Non-alcoholic fatty liver disease progresses to hepatocellular carcinoma in the absence of apparent cirrhosis. Int J Cancer 128, 2436-2443

Estes C, Razavi H, Loomba R, Younossi Z, Sanyal AJ (2017): Modeling the epidemic of nonalcoholic fatty liver disease demonstrates an exponential increase in burden of disease. Hepatology $\underline{67}, 123-133$

Feldman A, Eder SK, Felder TK, Kedenko L, Paulweber B, Stadlmayr A, Huber-Schönauer U, Niederseer D, Stickel F, Auer S (2017): Clinical and Metabolic Characterization of Lean Caucasian Subjects With Non-alcoholic Fatty Liver. Am J Gastroenterol 112, 102-110

Feldstein AE, Charatcharoenwitthaya P, Treeprasertsuk S, Benson JT, Enders FB, Angulo P (2009): The natural history of non-alcoholic fatty liver disease in children: a follow-up study for up to 20 years. Gut $\underline{58}, 1538-1544$

Feng R, Luo C, Li C, Du S, Okekunle AP, Li Y, Chen Y, Zi T, Niu Y (2017): Free fatty acids profile among lean, overweight and obese non-alcoholic fatty liver disease patients. A case control study. Lipids Health Dis $\underline{16}, 165$

Feng RN, Du SS, Wang C, Li YC, Liu LY, Guo FC, Sun CH (2014): Lean-non-alcoholic fatty liver disease increases risk for metabolic disorders in a normal weight Chinese population. World J Gastroenterol 20, 17932-17940

Fingas CD, Best J, Sowa JP, Canbay A (2016): Epidemiology of nonal-coholic steatohepatitis and hepatocellular carcinoma. Clin Liver Dis $\underline{8}, 119-122$

Fracanzani AL, Petta S, Lombardi R, Pisano G, Russello M, Consonni D, Di Marco V, Cammà C, Mensi L, Dongiovanni P (2017): Liver and Cardiovascular Damage in Patients With Lean 
Nonalcoholic Fatty Liver Disease, and Association With Visceral Obesity. Clin Gastroenterol Hepatol 15, 1604-1611

Gabrielli M, Moisan F, Vidal M, Duarte I, Jiménez M, Izquierdo G, Domínguez P, Méndez J, Soza A, Benitez C et al. (2012): Steatotic livers. Can we use them in OLTX? Outcome data from a prospective baseline liver biopsy study. Ann Hepatol $\underline{11}$, 891-898

Gastroenterological Society of Australia (GESA) (2013): Economic Cost and Health Burden of Liver Disease in Australia. https://www.gesa.org.au/resources/economic-cost-and-healthburden-of-liver-disease-in-australia/; abgerufen am 20.09.2019

Gatlin A (2016): Gilead Could Add \$1 Billion On NASH Drug, But Mechanism 'Puzzling'. Investor's Business Daily. https://www.investors.com/news/technology/gilead-could-add-1 billion-on-nash-drug-but-mechanism-puzzling/; abgerufen am 20.09.2019

Ghamar CME, Vahedi M, Pourhoseingholi MA, Ashtari S, Khedmat H, Amin M, Zali MR, Alavian SM (2013): Estimation of diagnosis and treatment costs of non-alcoholic Fatty liver disease: a two-year observation. Hepat Mon 13, e7382

Golabi P, Otgonsuren M, Cable R, Felix S, Koenig A, Sayiner M, Younossi ZM (2016): Nonalcoholic Fatty Liver Disease (NAFLD) is associated with im-pairment of Health Related Quality of Life (HRQOL). Health Qual Life Outcomes $\underline{14}, 18$

Gonzalez-Cantero J, Martin-Rodriguez JL, Gonzalez-Cantero A, Arrebola JP, Gonzalez-Calvin JL (2018): Insulin resistance in lean and overweight non-diabetic Caucasian adults. Study of its relationship with liver triglyceride content, waist circumference and BMI. PLoS One $\underline{9}$, e0192663

Greten TF, Malek NP, Schmidt S, Arends J, Bartenstein P, Bechstein W, Bernatik T, Bitzer M, Chavan A, Dollinger M et al. (2013): Diagnosis of and Therapy for Hepatocellular Carcinoma. Z Gastroenterol $\underline{51}, 1269-1326$

Gupta V, Mah XJ, Garcia MC, Antonypillai C, van der Poorten D (2015): Oily fish, coffee and walnuts. Dietary treatment for nonalcoholic fatty liver disease. World J Gastroenterol 21, 10621-10635

Gutiérrez-Grobe Y1, Chávez-Tapia N, Sánchez-Valle V, Gavilanes-Espinar JG, PoncianoRodríguez G, Uribe M, Méndez-Sánchez N (2012): High coffee intake is associated with lower grade nonalcoholic fatty liver disease. The role of peripheral antioxidant activity. Ann Hepatol 11, 350-355

Haenle M, Brockmann SO, Kron M, Bertling U, Mason RA, Steinbach G, Boehm BO, Koenig W, Kern P, Piechotowski I, Kratzer W (2006): Overweight, physical activity, tobacco and alcohol consumption in a cross-sectional random sample of German adults. BMC Public Health $\underline{6}, 233$

Hagström H, Nasr P, Ekstedt M, Kechagias S, Önnerhag K, Nilsson E, Rorsman F, Sheikhi R, Marschall HU, Hultcrantz R, Stål P (2017): Low to moderate lifetime alcohol consumption is associated with less advanced stages of fibrosis in non-alcoholic fatty liver disease. Scand J Gastroenterol 52,159-165 
Hagström H, Nasr P, Ekstedt M, Hammar U, Stål P, Hultcrantz R, Kechagias S (2018): Risk for development of severe liver disease in lean patients with nonalcoholic fatty liver disease. Hepatol Commun $\underline{30}$, 48-57

Hahn JM (Hrsg.): Checkliste Innere Medizin. 7. Auflage; Georg Thieme Verlag KG, Tübingen 2013

Hashemi Kani A, Alavian SM, Haghighatdoost F, Azadbakht L (2014): Diet macronutrients composition in nonalcoholic Fatty liver disease: a review on the related documents. Hepat Mon 14 , e10939

Hashimoto E, Tokushige K (2012): Hepatocellular carcinoma in non-alcoholic steatohepatitis: Growing evidence of an epidemic? Hepatol Res $\underline{42}, 1-14$

Hohenester S, Christiansen S, Nagel J, Wimmer R, Artmann R, Denk G, Bischoff M, Bischoff G, Rust C (2018): Lifestyle intervention for morbid obesity. Effects on liver steatosis, inflammation and fibrosis. Am J Physiol Gastrointest Liver Physiol 315, G329-G338

Holmer M, Melum E, Isoniemi H, Ericzon BG, Castedal M, Nordin A, Aagaard Schultz N, Rasmussen A, Line PD, Stål P, Bennet W, Hagström H (2018): Nonalcoholic fatty liver disease is an increasing indication for liver transplantation in the Nordic countries. Liver Int $\underline{38}, 2082$ 2090

Jayasekara H, English DR, Room R, MacInnis RJ (2014): Alcohol consumption over time and risk of death: a systematic review and meta-analysis. Am J Epidemiol 179, 1049-1059

Kabbany MN, Conjeevaram Selvakumar PK, Lopez R, Rayas MS, Lynch JL, Alkhouri N (2017): Prevalence of Nonalcoholic Steatohepatitis-Associated Cirrhosis in the United States: J Pediatr Gastroenterol Nutr $\underline{67}, 75-79$

Kagansky N, Levy S, Keter D, Rimon E, Taiba Z, Fridman Z, Berger D, Knobler H, Malnick S (2004): Non-alcoholic fatty liver disease--a common and benign finding in octogenarian patients. Liver Int 24, 588-594

Kälsch J, Bechmann LP, Heider D, Best J, Manka P, Kälsch H, Sowa JP, Moebus S, Slomiany U, Jöckel KH et al. (2015): Normal liver enzymes are correlated with severity of metabolic syndrome in a large population based cohort. Sci Rep $\underline{5}, 13058$

Kalthoff S, Ehmer U, Freiberg N, Manns MP, Strassburg CP (2010): Coffee induces expression of glucuronosyltransferases by the aryl hydrocarbon receptor and Nrf2 in liver and stomach. Gastroenterology 139, 1699-1710

Kanwal F, Kramer JR, Mapakshi S, Natarajan Y, Chayanupatkul M, Richardson PA, Li L, Desiderio R, Thrift AP, Asch SM et al. (2018): Risk of Hepatocellular Cancer in Patients with Nonalcoholic Fatty Liver Disease. Gastroenterology 155, 1828-1837

Katsagoni CN, Papatheodoridis GV, Ioannidou P, Deutsch M, Alexopoulou A, Papadopoulos N, Papageorgiou MV, Fragopoulou E, Kontogianni MD (2018): Improvements in clinical characteristics of patients with non-alcoholic fatty liver disease, after an intervention based on the Medi-terranean lifestyle. A randomised controlled clinical trial. Br J Nutr $\underline{120}$, 164-175 
Kawada N, Imanaka K, Kawaguchi T, Tamai C, Ishihara R, Matsunaga T, Gotoh K, Yamada T, Tomita Y (2009): Hepatocellular carcinoma arising from non-cirrhotic nonalcoholic steatohepatitis. J Gastroenterol 44, 1190-1194

Kennedy-Martin T, Bae JP, Paczkowski R, Freeman E (2017): Health-related quality of life burden of nonalcoholic steatohepatitis. A robust pragmatic literature review. J Patient Rep Outcomes $\underline{2}, 28$

Kim D, Choi SY, Park EH, Lee W, Kang JH, Kim W, Kim YJ, Yoon JH, Jeong SH, Lee DH et al. (2012): Nonalcoholic fatty liver disease is associated with coronary artery calcification. Hepatology $\underline{56}$, 605-613

Kim GA, Lee HC, Choe J, Kim MJ, Lee MJ, Chang HS, Bae IY, Kim HK, An J, Shim JH et al. (2017): Association between non-alcoholic fatty liver disease and cancer incidence rate. J Hepatol 8278, 32294-3229

Kim HJ, Kim HJ, Lee KE, Kim DJ, Kim SK, Ahn CW, Lim SK, Kim KR, Lee HC, Huh KB et al. (2004): Metabolic significance of nonalcoholic fatty liver disease in nonobese, nondiabetic adults. Arch Intern Med 164, 2169-2175

Kistler KD, Brunt EM, Clark JM, Diehl AM, Sallis JF, Schwimmer JB; NASH CRN Research Group (2011): Physical activity recommendations, exercise intensity, and histological severity of nonalcoholic fatty liver disease. Am J Gastroenterol 106, 460-468

Klatsky AL, Morton C, Udaltsova N, Friedman GD (2006): Coffee, cirrhosis, and transaminase enzymes. Arch Intern Med 166, 1190-1195

Koehler EM, Schouten JN, Hansen BE, Hofman A, Stricker BH, Janssen HL (2013): External validation of the fatty liver index for identifying nonalcoholic fatty liver disease in a population-based study. Clin Gastroenterol Hepatol 11, 1201-1204

Koopman KE, Caan MW, Nederveen AJ, Pels A, Ackermans MT, Fliers E, la Fleur SE, Serlie MJ (2014): Hypercaloric diets with increased meal frequency, but not meal size, in-crease intrahepatic triglycerides: a randomized controlled trial. Hepatology $\underline{60}, 545-553$

Kozakova M, Palombo C, Eng MP, Dekker J, Flyvbjerg A, Mitrakou A, Gastaldelli A, Ferrannini E; RISC Investigators (2012): Fatty liver index, gamma-glutamyltransferase, and early carotid plaques. Hepatology $\underline{55}, 1406-1415$

Kumar R, Mohan S (2017): Non-alcoholic Fatty Liver Disease in Lean Subjects. Characteristics and Implications. J Clin Transl Hepatol 28, 216-223

Kurth BM, Schaffrath Rosario A. (2007): Die Verbreitung von Übergewicht und Adipositas bei Kindern und Jugendlichen in Deutschland. Ergebnisse des bundesweiten Kinder- und Jugendgesund-heitssurveys (KiGGS). Bundesgesundheitsblatt Gesundheitsforschung Gesundheitsschutz 무, 736-43

Lazo M, Hernaez R, Eberhardt MS, Bonekamp S, Kamel I, Guallar E, Koteish A, Brancati FL, Clark JM (2013): Prevalence of nonalcoholic fatty liver disease in the United States: the Third National Health and Nutrition Examination Survey, 1988-1994. Am J Epidemiol 1, 38-45 
Le MH, Devaki P, Ha NB, Jun DW, Te HS, Cheung RC, Nguyen MH (2017): Prevalence of nonalcoholic fatty liver disease and risk factors for advanced fibrosis and mortality in the United States. PLoS One 12, e0173499

Lee JY, Kim KM, Lee SG, Yu E, Lim YS, Lee HC, Chung YH, Lee YS, Suh DJ (2007): Prevalence and risk factors of non-alcoholic fatty liver disease in potential living liver donors in Korea: a review of 589 consecutive liver biopsies in a single center. J Hepatol 47, 239-244

Lee YH, Kim KJ, Yoo ME, Kim G, Yoon HJ, Jo K, Youn JC, Yun M, Park JY, Shim CY et al. (2017): Association of nonalcoholic steatohepatitis with subclinical myocardial dysfunction in non-cirrhotic patients. J Hepatol $\underline{68}, 764-772$

Lee YI, Lim YS, Park HS (2012): Colorectal neoplasms in relation to non-alcoholic fatty liver disease in Korean women: a retrospective cohort study. J Gastroenterol Hepatol 27, 91-95

Leite NC, Salles GF, Araujo AL, Villela-Nogueira CA, Cardoso CR (2009): Prevalence and associated factors of non-alcoholic fatty liver disease in patients with type-2 diabetes mellitus. Liver Int 29, 113-119

Liu Y, Dai M, Bi Y, Xu M, Xu Y, Li M, Wang T, Huang F, Xu B, Zhang J et al. (2013): Active Smoking, Passive Smoking, and Risk of Nonalcoholic Fatty Liver Disease (NAFLD). A Population-Based Study in China. J Epidemiol 23, 115-121

Ma J, Hennein R, Liu C, Long MT, Hoffmann U, Jacques PF, Lichtenstein AH, Hu FB, Levy D (2018): Improved Diet Quality Associates With Reduction in Liver Fat, Particularly in Individuals With High Genetic Risk Scores for Nonalcoholic Fatty Liver Disease. Gastroenterology 155, 107-117

Malik SM, deVera ME, Fontes P, Shaikh O, Ahmad J (2009): Outcome after liver transplantation for NASH cirrhosis. Am J Transplant 2 , 782-793

Malik VS, Popkin BM, Bray GA, Després JP, Willett WC, Hu FB (2010): Sugar-sweetened beverages and risk of metabolic syndrome and type 2 diabetes: a meta-analysis. Diabetes Care $\underline{33}, 2477-2483$

Mantovani A, Zaza G, Byrne CD, Lonardo A, Zoppini G, Bonora E, Targher G (2017): Nonalcoholic Fatty Liver Disease Increases Risk of Incident Chronic Kidney Disease. A Systematic Review and Meta-Analysis. Metabolism $\underline{79}$, 64-76

Margariti A, Deutsch M, Manolakopoulos S, Tiniakos D, Papatheodoridis GV (2013): The severity of histologic liver lesions is independent of body mass index in patients with nonalcoholic fatty liver disease. J Clin Gastroenterol 47, 280-286

Margini C, Dufour JF (2016): The story of HCC in NAFLD. From epidemiology, across pathogenesis, to prevention and treatment. Liver Int $\underline{36}$, 317-324

Marmur J, Bergquist A, Stål P (2010): Liver transplantation of patients with cryptogenic cirrhosis: clinical characteristics and outcome. Scand J Gastroenterol 45, 60-69

Marventano S, Salomone F, Godos J, Pluchinotta F, Del Rio D, Mistretta A, Grosso G (2016): Coffee and tea consumption in relation with non-alcoholic fatty liver and metabolic syndrome. A systematic review and meta-analysis of observational studies. Clin Nutr $\underline{35}$, 1269-1281 
Mensink GB, Schienkiewitz A, Haftenberger M, Lampert T, Ziese T, Scheidt-Nave C (2013): Overweight and obesity in Germany: results of the German Health Interview and Examination Survey for Adults (DEGS1). Bundesgesundheitsblatt, Gesundheitsforschung, Gesundheitsschutz $\underline{56}, 786-794$

Merrell MD, Cherrington NJ (2011): Drug metabolism alterations in nonalcoholic fatty liver disease. Drug Metab Rev $\underline{43}, 317-334$

Molina-Molina E, Lunardi Baccetto R, Wang DQ, de Bari O, Krawczyk M, Portincasa P (2018): Exercising the hepatobiliary-gut axis. The impact of physical activity performance. Eur J Clin Invest $\underline{48}$, e12958

Molloy JW, Calcagno CJ, Williams CD, Jones FJ, Torres DM, Harrison SA (2012): Association of coffee and caffeine consumption with fatty liver disease, nonalcoholic steatohepatitis, and degree of hepatic fibrosis. Hepatology $\underline{55}$, 429-436

Mouzaki M, Comelli EM, Arendt BM, Bonengel J, Fung SK, Fischer SE, McGilvray ID, Allard JP (2013): Intestinal microbiota in patients with nonalcoholic fatty liver disease. Hepatology $\underline{58}$, $120-127$

Naderian M, Kolahdoozan S, Sharifi AS, Garmaroudi G, Yaseri M, Poustchi H, Sohrabpour AA (2017): Assessment of Lean Patients with Non-alcoholic Fatty Liver Disease in a Middle Income Country; Prevalence and Its Association with Metabolic Disorders: A Cross-sectional Study. Arch Iran Med 20, 211-217

Nishioji K, Sumida Y, Kamaguchi M, Mochizuki N, Kobayashi M, Nishimura T, Yamaguchi K, Itoh Y (2015): Prevalence of and risk factors for non-alcoholic fatty liver disease in a nonobese Japanese population, 2011-2012. J Gastroenterol 50, 95-108

Nobili V, Mosca A, De Vito R, Raponi M, Scorletti E, Byrne CD (2017): Liver zonation in children with NAFLD. Associations with dietary fructose and uric acid concentrations. Liver Int $\underline{38}$, 1102-1109

Oddy WH, Herbison CE, Jacoby P, Ambrosini GL, O'Sullivan TA, Ayonrinde OT, Olynyk JK, Black LJ, Beilin LJ, Mori TA et al. (2013): The Western dietary pattern is prospectively associated with nonal-coholic fatty liver disease in adolescence. Am J Gastroenterol 108, 778785

Okamoto M, Miyake T, Kitai K, Furukawa S, Yamamoto S, Senba H, Kanzaki S, Deguchi A, Koizumi M, Ishihara T et al. (2018): Cigarette smoking is a risk factor for the onset of fatty liver disease in nondrinkers. A longitudinal cohort study. PLoS One 13, e0195147

Ong JP, Pitts A, Younossi ZM (2008): Increased overall mortality and liver-related mortality in nonalcoholic fatty liver disease. J Hepatol 49, 608-612

Osaka T, Hashimoto Y, Hamaguchi M, Kojima T, Obora A, Fukui M (2018): Nonalcoholic fatty liver disease remission in men through regular exercise. J Clin Biochem Nutr $\underline{62}, 242-246$

Organisation For Economic Co-Operation And Development (OECD): Health at a Glance: Europe 2016: State of Health in the EU Cycle: Edition 2016. 1. Auflage; Oecd Publishing, Paris 2016 
Papandreou D, Karavetian M, Karabouta Z, Andreou E (2017): Obese Children with Metabolic Syndrome Have 3 Times Higher Risk to Have Nonalcoholic Fatty Liver Dise-ase Compared with Those without Metabolic Syndrome. Int J Endocrinol Article 2017, 2671692

Promrat K, Kleiner DE, Niemeier HM, Jackvony E, Kearns M, Wands JR, Fava JL, Wing RR (2010): Randomized controlled trial testing the effects of weight loss on nonalcoholic steatohepatitis. Hepatology $\underline{51}, 121-129$

Rajindrajith S, Pathmeswaran A Jayasinghe C, Kottahachchi D, Kasturiratne A, Silva ST, Niriella M, Dassanayake AS, Janaka H et al. (2017): Non-alcoholic fatty liver disease and its associations among adolescents in an urban, Sri Lankan community. BMC Gastroenterol 17, 135

Ratziu V, Bellentani S, Cortez-Pinto H, Day C, Marchesini G (2010): A position statement on NAFLD/NASH based on the EASL 2009 special conference. J Hepato. 53 , 372-384

Reddy SK, Marsh JW, Varley PR, Mock BK, Chopra KB, Geller DA, Tsung A (2012): Underlying steatohepatitis, but not simple hepatic steatosis, increases morbidity after liver resection: a case-control study. Hepatology $\underline{56}, 2221-2230$

Reid AE (2001): Nonalcoholic steatohepatitis. Gastroenterology 121, 710-723

Robinson SM, Wilson CH, Burt AD, Manas DM, White SA (2012): Chemotherapy-associated liver injury in patients with colorectal liver metastases: a systematic review and meta-analysis. Ann Surg Oncol 19, 4287-4299

Roeb E, Steffen HM, Bantel H, Baumann U, Canbay A, Demir M, Drebber U et al. (2015): Nichtalkoholische Fettlebererkrankungen; Version Januar 2015; Erstauflage. S2k-Leitlinie der deutschen Deutsche Gesellschaft für Gastroenterologie, Verdauungs- und Stoffwechselkrankheiten e.V. (DGVS) http://www.awmf.org/uploads/tx_szleitlinien/0210251_S25_NASH_Nicht_alkoholische_Fettlebererkrankung_2015-01.pdf.; abgerufen am 15.06.2019

Ryoo JH, Choi JM, Moon SY, Suh YJ, Shin JY, Shin HC, Park SK (2013): The clinical availability of non alcoholic fatty liver disease as an early predictor of the metabolic syndrome in Korean men: 5-year's prospective cohort study. Atherosclerosis $\underline{227}, 398-403$

Sanyal AJ (2018): Putting non-alcoholic fatty liver disease on the radar for primary care physi-cians. How well are we doing? BMC Med $\underline{16}, 148$

Sathiaraj E, Chutke M, Reddy MY, Pratap N, Rao PN, Reddy DN, Raghunath M. (2011): A casecontrol study on nutritional risk factors in non-alcoholic fatty liver disease in Indian population. Eur J Clin Nutr 도, 533-537

Schumann A, Hapke U, Rumpf HJ, Meyer C, John U (2001): The association between degree of nicotine dependence and other health behaviours. Findings from a German general population study. Eur J Public Health 11, 450-452

Schwimmer JB, Deutsch R, Kahen T, Lavine JE, Stanley C, Behling C (2006): Prevalence of fatty liver in children and adolescents. Pediatrics $\underline{118}, 1388-1393$ 
Shen H, Lipka S, Kumar A, Mustacchia P (2014): Association between nonalcoholic fatty liver disease and colorectal adenoma: a systemic review and meta-analysis. J Gastrointest Oncol $\underline{5}$, $440-446$

Shen H, Rodriguez AC, Shiani A, Lipka S, Shahzad G, Kumar A, Mustacchia P (2016): Association between caffeine consumption and nonalcoholic fatty liver disease. A systemic review and meta-analysis. Therap Adv Gastroenterol 9, 113-120

Shidfar F, Bahrololumi SS, Doaei S, Mohammadzadeh A, Gholamalizadeh M, Mohammadimanesh A (2018): The Effects of Extra Virgin Olive Oil on Alanine Aminotransferase, Aspartate Aminotransferase, and Ultrasonographic Indices of Hepatic Steatosis in Nonalcoholic Fatty Liver Disease Patients Undergoing Low Calorie Diet. Can J Gastroenterol Hepatol 17, 2018

Sinha RA, Farah BL, Singh BK, Siddique MM, Li Y, Wu Y, Ilkayeva OR, Gooding J, Ching J, Zhou J et al. (2014): Caffeine stimulates hepatic lipid metabolism by the autophagy-lysosomal pathway in mice. Hepatology $\underline{59}$, 1366-1380

Sookoian S, Pirola CJ, (2017): Systematic review with meta-analysis: risk factors for non-alcoholic fatty liver disease suggest a shared altered metabolic and cardiovascular profile between lean and obese patients. Aliment Pharmacol Ther 46, 85-95

Stadlmayr A, Aigner E, Steger B, Scharinger L, Lederer D, Mayr A, Strasser M, Brunner E, Heuberger A, Hohla F et al. (2011): Nonalcoholic fatty liver disease: an independent risk factor for colorectal neoplasia. J Intern Med 270, 41-49

Statistisches Bundesamt (2014): Rauchgewohnheiten nach Altersgruppen Ergebnisse des Mikrozensus 2017. Bonn. https://www.destatis.de/DE/ZahlenFakten/GesellschaftStaat/ Gesundheit/GesundheitszustandRelevantesVerhalten/Tabellen/Rauchverhalten.html; abgerufen am 15.06.2019

Stepanova M, Younossi ZM (2012): Independent association between nonalcoholic fatty liver disease and cardiovascular disease in the US population. Clin Gastroenterol Hepatol 10, 646-6

Stepanova M, De Avila L, Afendy M, Younossi I, Pham H; Cable R, Younossi ZM (2017): Direct and Indirect Economic Burden of Chronic Liver Disease in the United States. Clin Gastroenterol Hepatol 15; 759-766

Tamayo T, Brinks R, Hoyer A, Kuß OS, Rathmann W (2016): The Prevalence and Incidence of Diabetes in Germany. Dtsch Arztebl Int 113, 177-182

Tanajewski L, Harris R, Harman DJ, Aithal GP, Card TR, Gkountouras G, Berdunov V, Guha IN, Elliott RA (2017): Economic evaluation of a community-based diagnostic pathway to stratify adults for non-alcoholic fatty liver disease. A Markov model informed by a feasibility study. BMJ Open 7, e015659

Tannapfel A, Denk H, Dienes HP, Langner C, Schirmacher P, Trauner M, Flott-Rahmel B (2010): Histopathological diagnose of non-alcoholic and alcoholic fatty liver disease. Z Gastroenterol $\underline{48}, 486-98$ 
Tannapfel A, Denk H, Dienes HP, Langner C, Schirmacher P, Trauner M, Flott-Rahmel B (2011): Histopathological diagnosis of non-alcoholic and alcoholic fatty liver disease. Virchows Arch $\underline{458}, 511-523$

Tapper EB, Sengupta N, Hunink MG, Afdhal NH, Lai M (2015): Cost-Effective Evaluation of Nonalcoholic Fatty Liver Disease With NAFLD Fibrosis Score and Vibration Controlled Transient Elastography. Am J Gastroenterol 110, 1298-1304

Tapper EB, Hunink MG, Afdhal NH, Lai M, Sengupta N (2016): Cost-Effectiveness Analysis: Risk Stratification of Nonalcoholic Fatty Liver Disease (NAFLD) by the Primary Care Physician Using the NAFLD Fibrosis Score. PLoS One 11, e0147237

Thavorn K, Coyle D (2015): Transient Elastography and Controlled Attenuation Parameter for Diagnosing Liver Fibrosis and Steatosis in Ontario. An Economic Analysis. Ont Health Technol Assess Ser 15, 1-58

Thoma C, Day CP, Trenell MI (2012): Lifestyle interventions for the treatment of non-alcoholic fatty liver disease in adults: a systematic review. J Hepatol $\underline{56}$, 255-266

Treeprasertsuk S, Björnsson E, Enders F, Suwanwalaikorn S, Lindor KD (2013): NAFLD fibrosis score. A prognostic predictor for mortality and liver complications among NAFLD patients. World J Gastroenterol 19, 1219-1229

Utz-Melere M, Targa-Ferreira C, Lessa-Horta B, Epifanio M, Mouzaki M, Mattos AA (2018): NonAlcoholic Fatty Liver Disease in Children and Adolescents. Lifestyle Change - a Systematic Review and Meta-Analysis. Ann Hepatol 17, 345-354

Vanwagner LB, Bhave M, Te HS, Feinglass J, Alvarez L, Rinella ME (2012): Patients transplanted for nonalcoholic steatohepatitis are at increased risk for postoperative cardiovascular events. Hepatology $\underline{56}, 1741-1750$

Vernon G, Baranova A, Younossi ZM (2011): Systematic review: the epidemiology and natural history of non-alcoholic fatty liver disease and non-alcoholic steatohepatitis in adults. Aliment Pharmacol Ther $\underline{34}$, 274-285

Veronese N, Notarnicola M, Cisternino AM, Reddavide R, Inguaggiato R, Guerra V, Rotolo O, Zinzi I, Leandro G, Correale M et al. (2018): Coffee Intake and Liver Steatosis. A Population Study in a Mediterranean Area. Nutrients $\underline{10}$, e89

Vespasiani-Gentilucci U, Gallo P, Vincentis A, Galati G, Picardi A (2014): Hepatitis C virus and metabolic disorder interactions towards liver damage and atherosclerosis. World J Gastroenterol 2014 Mar 21, 2825-2838

Vlachopoulos C, Manesis E, Baou K, Papatheodoridis G, Koskinas J, Tiniakos D, Aznaouridis K, Archimandritis A, Stefanadis C (2010): Increased arterial stiffness and impaired endothelial function in nonalcoholic Fatty liver disease: a pilot study. Am J Hypertens 233, 1183-1189

Wang X, Li J, Riaz DR, Shi G, Liu C, Dai Y (2014): Outcomes of liver transplantation for nonalcoholic steatohepatitis: a systematic review and meta-analysis. Clin Gastroenterol Hepatol $\underline{12}, 394-402$ 
Wattacheril J, Sanyal AJ, (2016): Lean NAFLD: An Underrecognized Outlier. Curr Hepatol Rep 15, 134-139

Wijarnpreecha K, Thongprayoon C, Scribani M, Ungprasert P, Cheungpasitporn W (2017):

Noninvasive fibrosis markers and chronic kidney disease among adults with nonalcoholic fatty liver in USA. Eur J Gastroenterol Hepatol 30, 404-410

Wei JL, Leung JCF, Loong TCW, Wong GLH, Yeung DKW, Chan RSM, Shu SS, Chim AM, Chan AW, Choi PC, Tse YK, Chan HL, Wong VW (2015): Prevalence and Severity of Nonalcoholic Fatty Liver Disease in Non-Obese Patients. A Population Study Using Proton-Magnetic Resonance Spectroscopy. J Gastroenterol Hepatol 32, 1363-1369

Weiss J, Rau M, Geier A (2014): Non-alcoholic fatty liver disease: epidemiology, clinical course, investigation, and treatment. Dtsch Arztebl Int 111, 447-452

Welsh JA, Karpen S, Vos MB (2013): Increasing prevalence of nonalcoholic fatty liver disease among United States adolescents, 1988-1994 to 2007-2010. J Pediatr 162, 496-500

White DL, Kanwal F, El-Serag HB (2012): Association between nonalcoholic fatty liver disease and risk for hepatocellular cancer, based on systematic review. Clin Gastroenterol Hepatol 10, 1342-1359

Wieland AC, Quallick M, Truesdale A, Mettler P, Bambha KM (2013): Identifying practice gaps to optimize medical care for patients with nonalcoholic fatty liver disease. Dig Dis Sci $\underline{58}, 2809$ 2816

Wisocky J, Paul S (2017): The rising incidence of nonalcoholic fatty liver disease. Nurse Pract $\underline{42}$, $14-20$

Wong RJ, Cheung R, Ahmed A (2014): Nonalcoholic steatohepatitis is the most rapidly growing indication for liver transplantation in patients with hepatocellular carcinoma in the U.S.

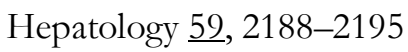

Wong RJ, Aguilar M, Cheung R, Perumpail RB, Harrison SA, Younossi ZM, Ahmed A (2015): Nonalcoholic steatohepatitis is the second leading etiology of liver disease among adults awaiting liver transplantation in the United States. Gastroenterology 148, 547-555

Wong S, Huynh D, Zhang F, Nguyen NQ, (2017): Use of aspartate aminotrans-ferase to platelet ratio to reduce the need for FibroScan in the evaluation of liver fibrosis. World J Hepatol $\underline{18}$, 791-796

Wong VW, Wong GL, Choi PC, Chan AW, Li MK, Chan HY, Chim AM, Yu J, Sung JJ, Chan HL (2010): Disease progression of non-alcoholic fatty liver disease: a prospective study with paired liver biopsies at 3 years. Gut $\underline{59}$, 969-974

Wong VW, Wong GL, Yip GW, Lo AO, Limquiaco J, Chu WC, Chim AM, Yu CM, Yu J, Chan FK et al. (2011): Coronary artery disease and cardiovascular outcomes in patients with nonalcoholic fatty liver disease. Gut $\underline{60}, 1721-1727$

Wong VW, Wong GL, Chan RS, Shu SS, Cheung BH, Li LS, Chim AM, Chan CK, Leung JK, Chu WC et al. (2018): Beneficial Effects of Lifestyle intervention in non-obese patients with nonalcoholic fatty liver disease. J Hepatol $\underline{69}$, 1349-1356 
World Health Organization (2000): Obesity - Preventing and Managing the Global Epidemic. Report on a WHO Consultation. Geneva. http://gbv.eblib.com/patron/FullRecord.aspx?p=284780; abgerufen am 15.06.2019

World Health Organization (2012): Adults in Europe consume three standard alcoholic drinks per day on average. Copenhagen. http://www.euro.who.int/en/media-centre/sections/pressreleases/2012/03/adults-in-europe-consume-three-standard-alcoholic-drinks-per-day-onaverage; abgerufen am 21.09.2019

World Health Organization (2014): Global status report on alcohol and health. 2014. Luxembourg. https://apps.who.int/iris/bitstream/handle/10665/112736/9789240692763_eng.pdf;jsessioni $\mathrm{d}=409183411 \mathrm{~B} 112 \mathrm{C} 6 \mathrm{~F} 2958 \mathrm{~B} 859 \mathrm{~B} 8 \mathrm{~B} 17679$ ?sequence=1; abgerufen am 21.09.2019

World Medical Association (2018): WMA Declaration of Helsinki - Ethical Principles for Medical Research Involving Human Subjects. https://www.wma.net/policies-post/wma-declarationof-helsinki-ethical-principles-for-medical-research-involving-human-subjects/; abgerufen am 20.09.2019

Xu C, Yu C, Ma H, Xu L, Miao M, Li Y (2013): Prevalence and risk factors for the development of nonalcoholic fatty liver disease in a nonobese Chinese population. The Zhejiang Zhenhai Study. Am J Gastroenterol 108, 1299-1304

Yatsuji S, Hashimoto E, Tobari M, Taniai M, Tokushige K, Shiratori K (2009): Clinical features and outcomes of cirrhosis due to non-alcoholic steatohepatitis com-pared with cirrhosis caused by chronic hepatitis C. J Gastroenterol Hepatol 24, 248-254

Yeh M, Brunt EM (2014): Pathological features of fatty liver disease. Gastroenterology 147, 754 764

Yesil A, Yilmaz Y (2013): Review article. Coffee consumption, the metabolic syndrome and nonalcoholic fatty liver disease. Aliment Pharmacol Ther $\underline{38}, 1038-1044$

Yoshitaka H, Hamaguchi M, Kojima T, Fukuda T, Ohbora A, Fukui M (2017): Nonoverweight nonalcoholic fatty liver disease and incident cardiovascular disease: A post hoc analysis of a cohort study. Medicine $\underline{96}$, e6712

Younossi ZM, Stepanova M, Negro F, Hallaji S, Younossi Y, Lam B, Srishord M (2012): Nonalcoholic fatty liver disease in lean individuals in the United States. Medicine 11, 319-327

Younossi ZM, Zheng L, Stepanova M, Henry L, Venkatesan C, Mishra A (2015): Trends in outpatient resource utilizations and outcomes for Medicare beneficiaries with nonalcoholic fatty liver disease. J Clin Gastroenterol 49, 222-227

Younossi ZM, Blissett D, Blissett R, Henry L, Stepanova M, Younossi Y, Racila A, Hunt S, Beckerman R (2016): The economic and clinical burden of nonalcoholic fatty liver disease in the United States and Europe. Hepatology 64, 1577-1586

Younossi ZM, Anstee QM, Marietti M, Hardy T, Henry L, Eslam M (2018a): Global burden of NAFLD and NASH. Trends, predictions, risk factors and prevention. Nat Rev Gastroenterol Hepatol 15 , 11-20 
Younossi ZM, Stepanova M, Ong JP, Jacobson IM, Bugianesi E, Duseja A, Eguchi Y, Wong VW, Negro F, Yilmaz Y et al. (2018b): Non-alcoholic Steatohepatitis is the Fastest Growing Cause of Hepatocellular Carcinoma in Liver Transplant Candidates. Clin Gastroenterol Hepatol 17, 748-755

Zeiher J, Kuntz B, Lange C (2017): Rauchen bei Erwachsenen in Deutschland. J Health Monit 2, $59-65$

Zein CO, Unalp A, Colvin R, Liu YC, McCullough AJ, (2011): Smoking and severity of hepatic fibrosis in nonalcoholic fatty liver disease. J Hepatol $\underline{54}, 753-759$

Zelber-Sagi S, Nitzan-Kaluski D, Goldsmith R, Webb M, Blendis L, Halpern Z, Oren R (2007): Long term nutritional intake and the risk for non-alcoholic fatty liver disease (NAFLD). A population based study. J Hepatol $\underline{47}, 711-717$

Zelber-Sagi S, Nitzan-Kaluski D, Goldsmith R, Webb M, Zvibel I, Goldiner I, Blendis L, Halpern Z, Oren R (2008): Role of leisure-time physical activity in nonalcoholic fatty liver disease: a popula-tion-based study. Hepatology $\underline{48}, 1791-1798$

Zelber-Sagi S, Lotan R, Shlomai A, Webb M, Harrari G, Buch A, Nitzan Kaluski D, Halpern Z, Oren R (2012): Predictors for incidence and remission of NAFLD in the general population during a seven-year prospective follow-up. J Hepatol $\underline{56}$, 1145-1151

Zelber-Sagi S, Lotan R, Shibolet O, Webb M, Buch A, Nitzan-Kaluski D, Halpern Z, Santo E, Oren R (2013): Non-alcoholic fatty liver disease independently predicts prediabetes during a 7 year prospective follow-up. Liver Int $\underline{33}, 1406-1412$

Zezos P, Renner EL (2014): Liver transplantation and non-alcoholic fatty liver disease. World J Gastroenterol 20, 15532-15538

Zhang E, Wartelle-Bladou C, Lepanto L, Lachaine J, Cloutier G, Tang A (2015): Cost-utility analysis of nonalcoholic steatohepatitis screening. Eur Radiol 25, 3282-3294

Zheng X, Gong L, Luo R, Chen H, Peng B, Ren W, Wang Y (2017): Serum uric acid and nonalcoholic fatty liver disease in non-obesity Chinese adults. Lipids Health Dis $\underline{16}, 202$

Zhou YJ, Li YY, Nie YQ, Huang CM, Cao CY (2012): Natural course of nonalcoholic fatty liver disease in southern China: a prospective cohort study. J Dig Dis 13, 153-160

Zou TT, Zhang C, Zhou YF, Han YJ, Xiong JJ, Wu XX, Chen YP, Zheng MH (2018): Lifestyle interventions for patients with nonalcoholic fatty liver disease. A network meta-analysis. Eur J Gastroenterol Hepatol $\underline{30}$, 747-755 


\section{Danksagung}

Mein persönlicher Dank gilt der Klinik für Gastroenterologie und gastrointestinale Onkologie der Universitätsmedizin Göttingen unter Führung von Prof. Dr. Volker Ellenrieder für die grundlegende Ermöglichung des gesamten Projektes. Ein bersonmderer Dank gilt meinen Betreuern PD Dr. med. Steffen Kunsch bzw. PD Dr. med. Thomas Lorf für Ihre stetige Unterstützung, wissenschaftliche Begleitung des Projektes und die regelmäßige Diskussion der Fortschritte bzw. Ergebnisse.

Explizit hinweisen möchte ich auf die enge Unterstützung in methodischer, wissenschaftlicher und planerischer Hinsicht durch Dr. med. Sebastian Bremer, der die Arbeiten an diesem Projekt über den gesamten Zeitraum maßgeblich mitbetreute und gefördert hatsowie stetig wesentliche Impulse zur Projektentwicklung mit einbrachte. Nicht zuletzt die Vermittlung wissendschaftlicher Hintergründe und praktischer Erfahrungen führten für mich $\mathrm{zu}$ einem tieferen Verständnis der theoretischen und praktischen Hintergründe. Auch möchte ich Dr. med. Lars Reinhardt für seine Unterstützung in der Datenerhebung und inhaltlichen Aus- und Weiterbildung meinen Dank aussprechen.

Den nicht-ärztlichen Mitarbeitern des Leberzentrums der Universitätsklinik Göttingen, hier insbesondere Frau Alexandra Nau und Frau Kathrin Lesemann, gilt besonderer Dank für die organisatorische und logistische Unterstüzung dieses Projektes und die nette Aufnahme in das Team. 